\title{
SIMULATION OF FLUID FLOW DYNAMICS IN DESIGNING A BI-VENTRICULAR ASSIST DEVICE USING CFD TOOLS
}

\author{
By \\ Lamiya Sadaf \\ 19121158 \\ Walia Hoque \\ 15321014 \\ Nazifa Tabassum Zaima \\ 16121101 \\ Sadiah Karim \\ 16121144
}

\begin{abstract}
A Thesis submitted to the Department of Electrical and Electronic Engineering Of BRAC University, in partial fulfillment of the requirements for the degree of Bachelor of Science in Electrical and Electronic Engineering
\end{abstract}

Department of Electrical and Electronic Engineering

Brac University

August, 2019

(C) [2019]. Brac University

All rights reserved 


\section{Declaration}

It is hereby declared that,

1. The thesis submitted is my/our own original work while completing degree at BRAC University.

2. The thesis does not contain material previously published or written by a third party, except where this is appropriately cited through full and accurate referencing.

3. The thesis does not contain material which has been accepted, or submitted, for any other degree or diploma at a university or other institution.

4. We have acknowledged all main sources of help.

Student's Full Name \& Signature:

Lamiya Sadaf

19121158
Walia Hoque

15321014
Nazifa Tabassum Zaima

16121101
Sadiah Karim

16121144 


\section{Approval}

The thesis/project titled "Simulation of Fluid Flow Dynamics in Designing a Bi-Ventricular Assist Device Using CFD Tools" submitted by

Lamiya Sadaf (ID-19121158)

Walia Hoque (ID-15321014)

Sadiah Karim (ID-16121144)

Nazifa Tabassum Zaima (ID-16121101)

Of Summer, 2019 has been accepted as satisfactory in partial fulfillment of the requirement for the degree of Bachelors of Engineering in Electrical and Electronic Engineering on $29^{\text {th }}$ August, 2019.

\section{Examining Committee:}

Supervisor:

(Member)

A. K. M. Abdul Malek Azad, PhD

Professor, Department of Electrical and Electronic Engineering BRAC University

Program Coordinator:

(Member)

Saifur Rahman Sabuj, PhD Assistant Professor, Department of Electrical and Electronic Engineering BRAC University

Departmental Head:

(Chair)

Shahidul Islam Khan, PhD Professor and Chairperson, Department of Electrical and Electronic Engineering BRAC University 


\begin{abstract}
One of the main worldwide health worries now consists of cardiovascular diseases due to its fatality and the swiftly growing number of identified sufferers. Severe cases result in heart failures that require heart transplantations which are restricted by very limited number of available donor hearts. Henceforth, a Ventricular Assist Device (VAD) has been an advance development to deal with the heart scarcity problem by offering support to sustain patients while they are expecting donor hearts to become available, or even to use as a lifelong assistance if transplantation is not feasible. However, implementation of this device might result in damage to the blood cells; or in the worst-case scenario, mechanical failures can take place especially for those patients who suffered Bi-Ventricular heart failures. In consideration to the stated problems and to increase both the reliability and functioning of the device, the concept design of an innovative Bi-VAD with the help of computational fluid dynamics (CFD) is proposed in this thesis. The thesis aims to use CFD as the main designing tool to analyze flow and pump performance and interpret the hemodynamic and hydrodynamic risks associated with the preliminary stage of development. The thesis intends to find out the best design of the centrifugal pump that can be executed in experimental evaluation followed by human mock circulation in actual operating conditions.
\end{abstract}

The accomplished development of the heart pump and CFD analysis technique will have a hand in the heart pump construction by not only enhancing its performance but also reducing associated risks of complications in the design resulting in reduced lead time and cost of production. Further research is necessary that includes experimental implementation of the designs and henceforth animal and clinical trials before it is ready to support Bi-Ventricular failure patients giving them a light of hope apart from heart transplantation.

Keywords: VAD; Bi-VAD; CFD. 


\section{Dedication}

We would like to dedicate our thesis to our parents for always encouraging us to strive for

excellence and for their continuous love and support during every phase of our lives without which we could not have come so far. 


\section{Acknowledgement}

We would like to thank Dr. A K M Abdul Malek Azad, Professor, Department of Electrical and Electronics Engineering (EEE), BRAC University; for his constant support, guidance and valuable opinions during this entire phase which led us to successfully completing this thesis. We are also grateful to BRAC University for providing us with all the necessary materials required for the successful execution of our thesis. 


\section{Table of Contents}

Declaration...............................................................................................................................

Approval .................................................................................................................................................................. iii

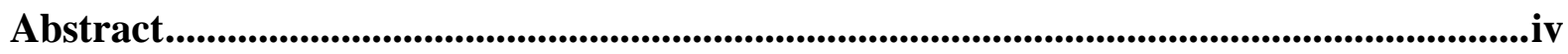

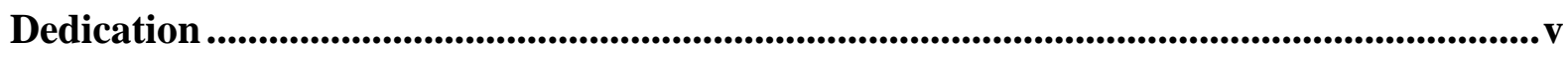

Acknowledgement .............................................................................................................................................vi

Table of Contents ...............................................................................................................................................vii

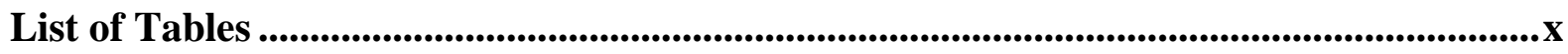

List of Figures.................................................................................................................................................... xiii

List of Acronyms ..............................................................................................................................................

Chapter 1: Introduction .............................................................................................1

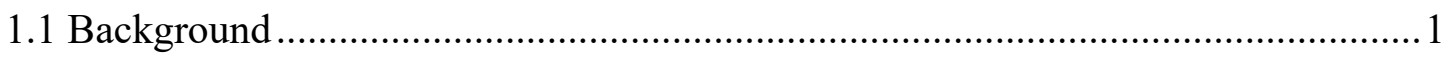

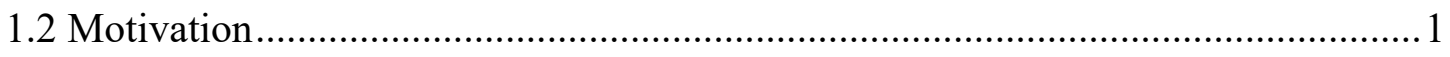

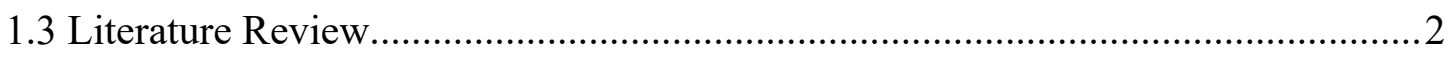

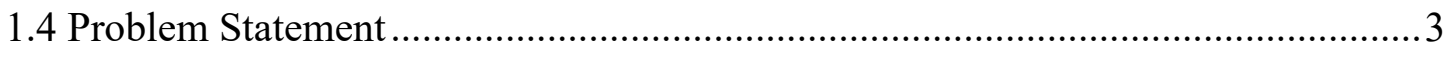

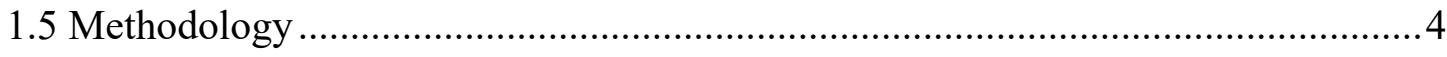

1.6 Overview of the Contents ..................................................................................

Chapter 2: Thesis Overview.............................................................................................................................8

2.1 Design of Impeller .......................................................................................

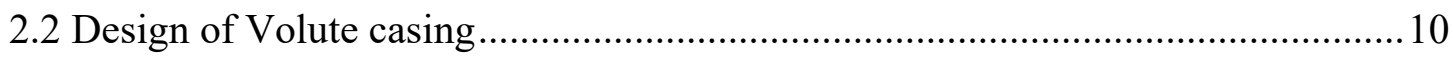

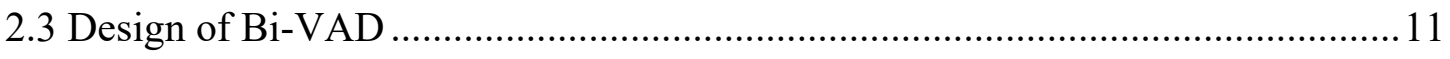

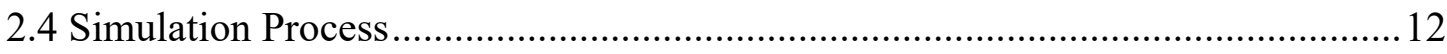

Chapter 3: Mathematical Modeling for CFD analysis of the Pump .....................................13

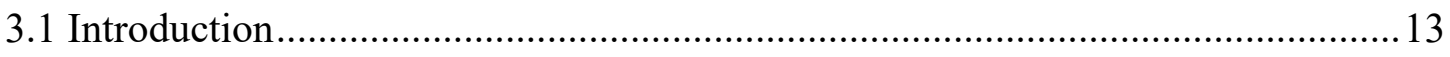

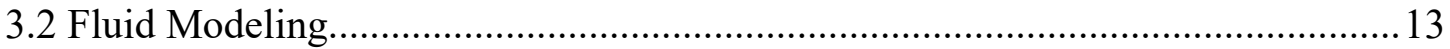


3.3 Flow Modeling .................................................................................................. 14

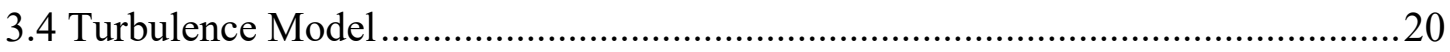

3.5 Blood Damage Model .........................................................................................28

Chapter 4: Design and Simulation of Ventricular Assist Devices (VADs) ..........................35

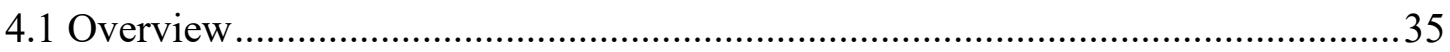

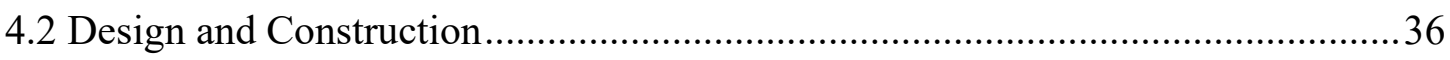

4.2.1 VAD Design Background .......................................................................

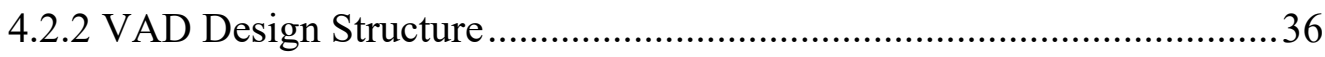

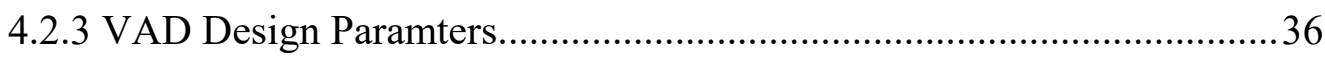

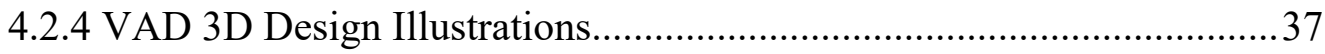

4.3 Initial Single Sided VAD Simulation ..............................................................41

4.3.1 Geometry Establishment ........................................................................41

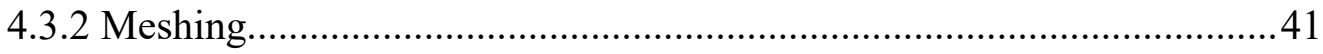

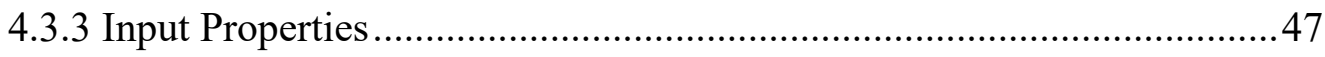

4.3.4 Boundary Conditions ............................................................................. 4

4.3.5 Setup and Discussions ......................................................................52

4.3.6 Selection of Best Impeller for RVAD and Bi-VAD simulation ..............53

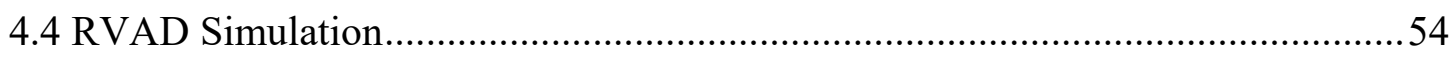

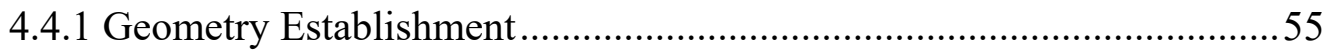

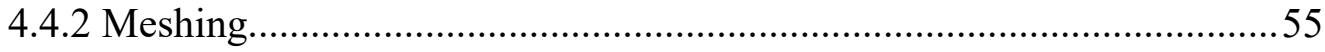

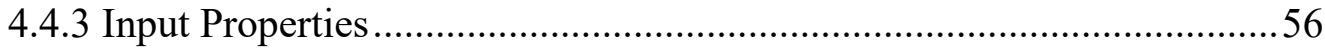

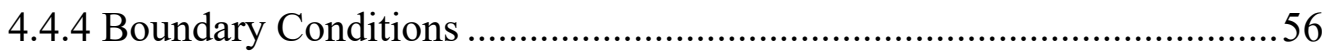

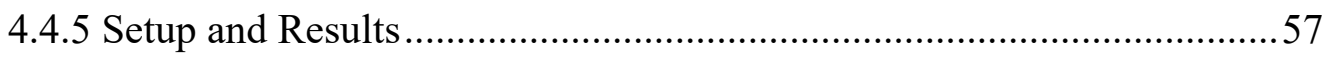

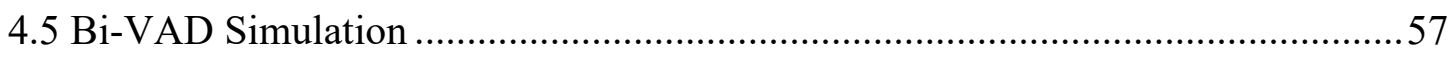

4.5.1 Geometry Establishment ....................................................................5 


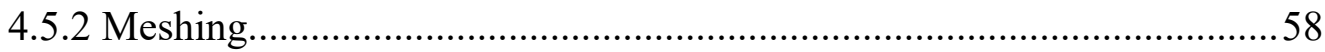

4.5.3 Input Properties ..........................................................................5

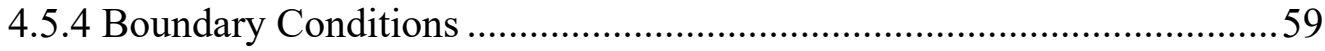

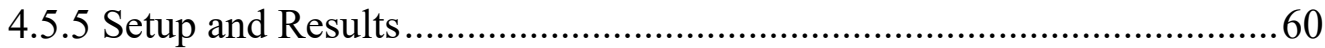

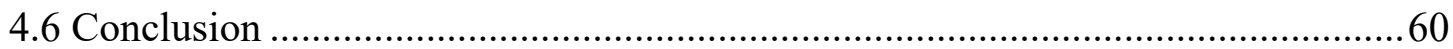

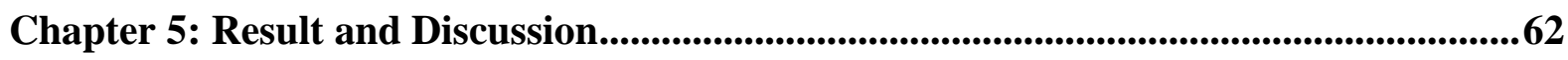

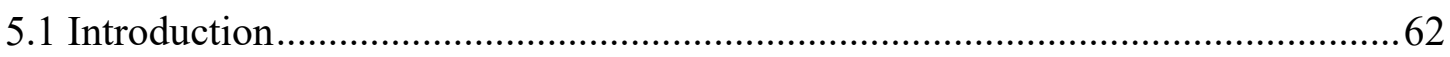

5.2 Required equations for fluid flowing mechanism........................................63

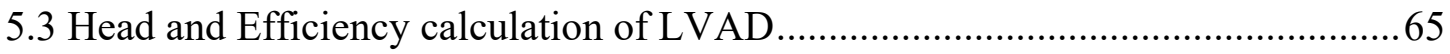

5.4 Graphical representations for comparison of LVAD Impellers.........................73

5.5 Conclusion on ultimate best Impeller design of LVAD ................................... 76

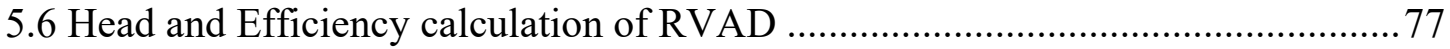

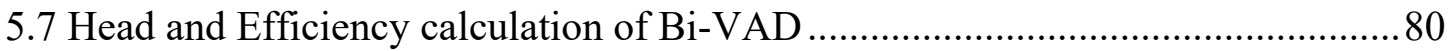

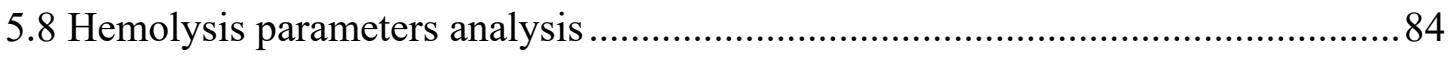

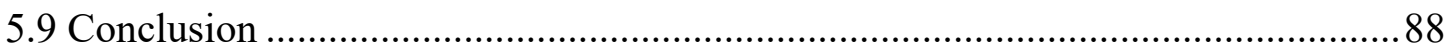

Chapter 6: Conclusion .................................................................................................................................89

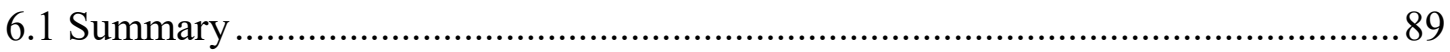

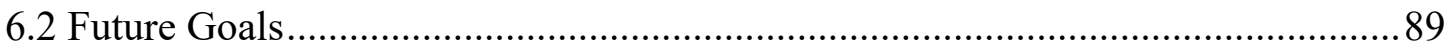

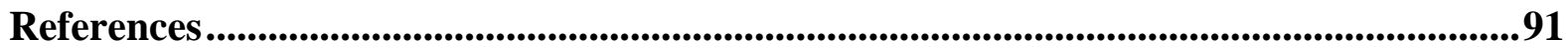

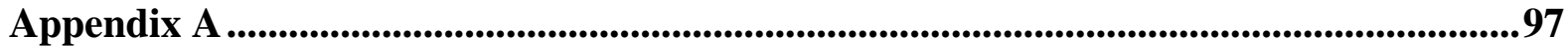

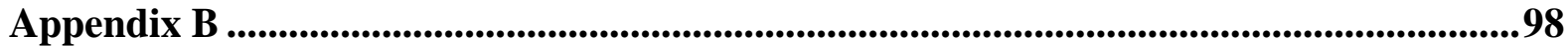




\section{List of Tables}

Table 4.1 - List of design parameters of the VAD pump 37

Table 4.2 - The mesh sizes varied for Impeller $1\left(100^{\circ}\right.$ wrap angle and 6 blades $)$ and the resulting pressure difference and torque obtained from simulation along with the calculated Head and Efficiency

Table 4.3 - The mesh sizes varied for Impeller2 $\left(100^{\circ}\right.$ wrap angle and 5 blades $)$ and the resulting pressure difference and torque obtained from simulation along with the calculated Head and Efficiency

Table 4.4 - The mesh sizes varied for Impeller3 (110 wrap angle and 6 blades) and the resultant pressure difference and torque obtained from simulation along with the calculated Head and Efficiency

Table 4.5- The element sizes varied for Impeller4 $\left(110^{\circ}\right.$ wrap angle and 5 blades $)$ and the resultant pressure difference and torque obtained from simulation along with the calculated Head and Efficiency

Table 4.6 - The element sizes varied for Impeller5 ( $120^{\circ}$ wrap angle and 6 blades) and the resultant pressure difference and torque obtained from simulation along with the calculated Head and Efficiency

Table 4.7 - The mesh sizes varied for Impeller6 (120 wrap angle and 5 blades) and the resultant pressure difference and torque along with the calculated Head and Efficiency 45

Table 4.8 - Calculated Head and Efficiency at different Flow rates for corresponding Pressure Difference and Torque of Impeller1 with $100^{\circ}$ wrap angle and 6 blades .48

Table 4.9 - Calculated Head and Efficiency at different Flow rates for corresponding Pressure Difference and Torque of Impeller2 with $100^{\circ}$ wrap angle and 5 blades 49

Table 4.10 - Calculated Head and Efficiency at different Flow rates for corresponding Pressure Difference and Torque of Impeller 3 with $110^{\circ}$ wrap angle and 6 blades

Table 4.11 - Calculated Head and Efficiency at different Flow rates for corresponding Pressure Difference and Torque of Impeller4 with $110^{\circ}$ wrap angle and 5 blades 50 
Table 4.12 - Calculated Head and Efficiency at different Flow rates for corresponding Pressure Difference and Torque of Impeller5 with $120^{\circ}$ wrap angle and 6 blades 50

Table 4.13 - Calculated Head and Efficiency at different Flow rates for corresponding Pressure Difference and Torque of Impeller6 with $120^{\circ}$ wrap angle and 5 blades 51

Table 4.14 - Summary Table of Head and Efficiency of Impeller design 1 to 6 at optimum mesh size of $0.30 \mathrm{~mm}$ and flow rate of $0.30 \mathrm{~m}^{3} / \mathrm{h}$ 54

Table 4.15 - Calculated Head and Efficiency of RVAD for corresponding Pressure Difference and Torque of Impeller1 with $100^{\circ}$ wrap angle and 6 blades .

Table 4.16 - Calculated Head and Efficiency of BIVAD for corresponding Pressure Difference and Torque of Best Impeller design with $100^{\circ}$ wrap angle and 6 blades 60

Table 5.1 - Obtained Values of Pressure difference, $\Delta P$ and Torque, $\tau$ for all the designed Impellers (i.e., Impeller1, 2, 3, 4, 5, 6) run with the best value of mesh size (i.e., $0.30 \mathrm{~mm}$ ) to be used for calculation of Head and Efficiency 66

Table 5.2 - The final results table with the calculated values of Head and Efficiency for the corresponding value of Pressure difference and Torque for the Impeller designs with the best mesh size

Table 5.3 - The obtained values of Pressure difference, $\Delta P$ and Torque, $\tau$ for Impeller1 being simulated with different Mass Flow rates and the best mesh size of $0.3 \mathrm{~mm}$ 71

Table 5.4 - The calculated values of Head and Efficiency for the Pressure difference and Torque for Impeller1 being simulated for different Mass Flow rates and mesh size of $0.3 \mathrm{~mm}$ 73

Table 5.5 - Obtained values of Pressure difference, $\Delta P$ and Torque, $\tau$ for RVAD upon simulation with conditions of $0.3 \mathrm{~mm}$ mesh size, $0.3 \mathrm{~m}^{3} / \mathrm{h}$ Flow rate and an angular frequency of rotation having a value of $3054 \mathrm{rpm}$ 78

Table 5.6 - The calculated values of Head and Efficiency for the corresponding values of Pressure difference and Torque for RVAD with the operating conditions for RVAD described earlier 80

Table 5.7 (a) - The calculated values of Head and Efficiency for the corresponding Pressure difference and Torque for the best design of LVAD under operating conditions of $0.30 \mathrm{~m}^{3} / \mathrm{h}$ 
Flow rate and mesh size of $0.30 \mathrm{~mm}$ and Mass Flow rate of $0.088 \mathrm{~kg} / \mathrm{s}$ before being conjoined together 80

Table 5.7 (b) - The calculated values of Head and Efficiency for the corresponding Pressure difference and Torque for the best design of RVAD simulated with the best design of LVAD under operating conditions of $0.30 \mathrm{~m}^{3} / \mathrm{h}$ Flow rate and mesh size of $0.30 \mathrm{~mm}$ and Mass Flow rate of $0.088 \mathrm{~kg} / \mathrm{s}$ before being conjoined together.

Table 5.8 - The obtained Pressure difference, $\Delta P$ and Torque, $\tau$ for LVAD and RVAD separately upon simulation of $\mathrm{Bi}-\mathrm{VAD}$ 


\section{List of Figures}

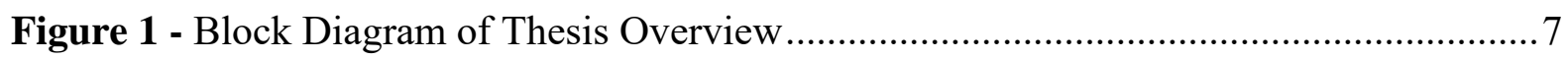

Figure 2.1 - Visual Demonstration of how a Bi-VAD is installed in a Human Heart ..............9

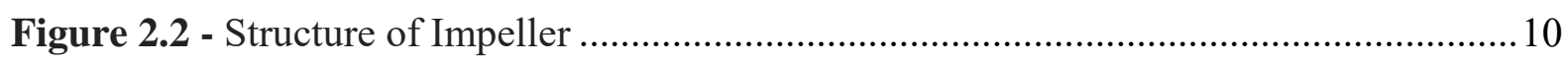

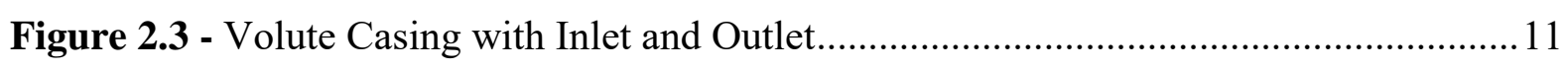

Figure 2.4 (a) - Volute casing of the inlet and outlet of BIVAD model ..................................12

Figure 2.4 (b) - Impeller of pump with Volute casing........................................................ 12

Figure 4.1 (a) - Impeller with $100^{\circ}$ wrap angle with 5 blades ................................................

Figure 4.1 (b) - Impeller with $100^{\circ}$ wrap angle with 6 blades................................................37

Figure 4.2 (a) - Impeller with $110^{\circ}$ wrap angle with 6 blades ...............................................38

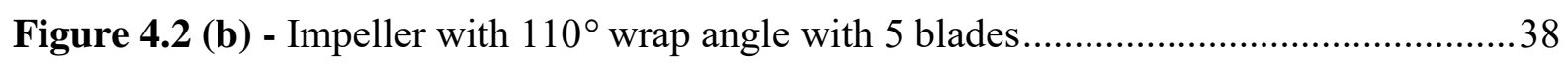

Figure 4.3 (a) - Impeller with $120^{\circ}$ wrap angle with 5 blades ............................................... 38

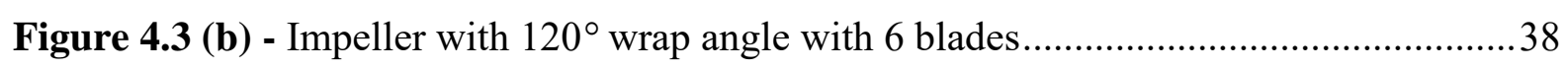

Figure 4.4 (a) - Impeller with casing and eye of 6 blades .....................................................39

Figure 4.4 (b) - Impeller with casing and eye of 5 blades ...................................................39

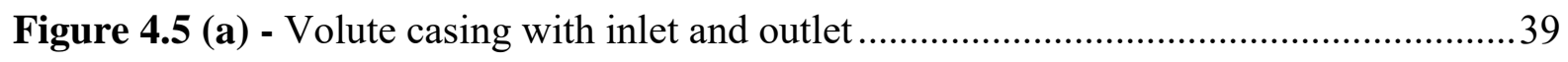

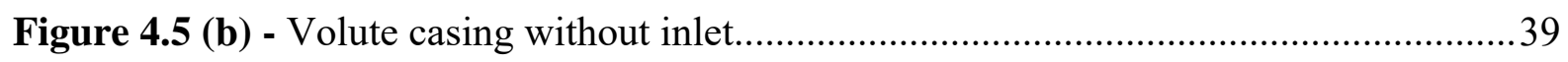

Figure 4.6 (a) - Impeller of 5 blades with volute casing ....................................................40

Figure 4.6 (b) - Impeller of 6 blades with volute casing.....................................................40

Figure 4.7 (a) - Volute casing of inlet and outlet of BIVAD model.....................................40

Figure 4.7 (b) - Impeller of the best design $\left(100^{\circ}\right.$ wrap angle with 6 blades $)$ with Volute casing

Figure 4.8 - Volute casing mesh used for the 6 impeller designs ...........................................45

Figure 4.9 - Zoomed in volute casing to observe mesh ......................................................46 
Figure 4.10 (a) - Impeller mesh design for 5 blades ............................................. 46

Figure 4.10 (b) - Impeller mesh design for 6 blades......................................................46

Figure 4.11 - Interface at the connecting surface between rotating and stationary domain ...51

Figure 4.12 - RVAD geometry model of the best design of Impeller1 with $100^{\circ}$ wrap angle and 6 blades 55

Figure 4.13 - RVAD mesh model of the best design of Impeller1 with $100^{\circ}$ wrap angle and 6 blades 56

Figure 4.14 - Final Bi-VAD geometry model of the best design.......................................58

Figure 4.15 - Final Bi-VAD mesh model of the best design ..... 59

Figure 5.1 - The above diagram illustrates the visual representation of the Mass Flow rates Vs Efficiency for all the designed Impellers for LVAD (i.e., Impeller1, 2, 3, 4, 5 and 6) 74

Figure 5.2 - The above graph illustrates the bar graph representation of Head and corresponding Efficiency for all the 6 designed Impellers of LVAD (i.e., Impeller1, 2, 3, 4, 5 and 6) at a mesh size of $0.3 \mathrm{~mm}$. 75

Figure 5.3 - The graph illustrates the graphical form of the Head Vs Mass Flow rate of the 6 designed Impellers of LVAD (i.e., Impeller1, 2, 3, 4, 5, 6) 75

Figure 5.4 - Impeller1 pressure, velocity and shear stress profile 85

Figure 5.5 - Impeller2 pressure, velocity and shear stress profile 85

Figure 5.6 - Impeller3 pressure, velocity and shear stress profile .86

Figure 5.7 - Impeller4 pressure, velocity and shear stress profile 86

Figure 5.8 - Impeller5 pressure, velocity and shear stress profile 87

Figure 5.9 - Impeller6 pressure, velocity and shear stress profile 87 


\section{List of Acronyms:}

LVAD - Left Ventricular Assist Device

RVAD - Right Ventricular Assist Device

Bi-VAD - Bi-Ventricular Assist Device

CFD - Computational Fluid Dynamics

RBC - Red Blood Cell

SS - Shear Stress 


\section{Chapter 1}

\section{Introduction}

\subsection{Background}

A ventricular assist device (VAD) is a mechanical pump that can be implemented in the heart which helps to pump blood from the lower chambers of the heart known as ventricles to the whole body. VADs are also referred to as mechanical circulatory support devices. VADs can further be categorized into the Left Ventricular assist device usually termed as LVAD and Right Ventricular assist device commonly termed as RVAD. Left Ventricular assist devices are most commonly used ones. In cases where there is a failure in both the ventricles, the Bi-Ventricular Assist Device termed as Bi-VAD can also be implanted. While heart transplantation is the most effective and reliable solution of severe cases of heart failure, VADs can play a very vital function in offering both long term as well as short-term support to the patients. The main obstacle of heart transplantation is the scarcity of donor hearts; implantation of VAD can solve this problem to a great extent. One can be recommended for VAD transplantation even as looking forward for a donor heart to be available. In cases of temporary heart failure, the VAD can be embedded until the heart returns to its normal function. However, the patient may not always be eligible for a heart transplant due to age or other scientific medical conditions. VADs can be used as a permanent treatment and provide long term support to the patient. However, there are also some risks associated with implantation of VAD such as blood clots, blood damage, infection, mechanical failure etc. Many researches such as ours are working to improve the quality of VADs by solving these problems. HeartMateII, HeartMateIII, Jarvik 2000, Micromed DeBakey VAD, HVAD, MVAD etc. are some currently existing and used Ventricular Assist Devices.

\subsection{Motivation}

Heart failure is one of the most growing fatal cardiovascular diseases with a rapid growth, which makes it a global warning concern. Though there are numerous treatments available relying on the depth of the heart failure, the most effective and dependable technique for severe cases are 
heart transplants. Due to the dearth of donor hearts, heart transplants cannot be performed most of the instances. As a result, only a small portion of patients receive transplantations each year. Ventricular Assist Devices are developed to cope up with the donor heart shortage hassle by supplying short term support to the patients expecting donor heart or even to use as a permanent support if heart transplantation is not possible. Clinical studies have proven the usefulness of these devices as a permanent solution as they bring forth improvements in the quality of life and increases the survival rate compared to the highest quality medical treatment [1]. However, there are some design complications which reduces the lifespan of VADs. Durability and hemodynamic performance are one of the major concerns of VAD design which includes blood clots, blood damage, thrombosis and mechanical failure. On the other hand, there is a lack of BiVentricular assist devices. Most of the VADs are designed for Left Ventricular guide in the view that Left Ventricular failure is the most usual form of heart failure. In case of biventricular failure installment of two different devices adds complexity in control unit and additional driveline. Moreover, requires more space in heart, which is not convenient for women and children. Therefore, unfolding of the development of a Bi-Ventricular assist device (Bi-VAD) in a single module is very essential in order to support the Bi-Ventricular failure patients. In the light of the above-mentioned problems, this work was chosen with a vision of designing a BiVAD with better Efficiency, Durability and Hemodynamic performance.

\subsection{Literature Review}

In [2] a comparative study between pulsatile pump and continuous flow pumps has been discussed. In [2] it has been discussed that due to pulsatile pump the failure of VAD. After intensive research it has been found that continuous flow pumps are better. Using this people can survive a good 4-5 years than by using the pulsatile pump. Along with that it has better Efficiency than the former. In [3] the purpose is through CFD to assess hemodynamics in animal models. In [1] through CFD, mechanism of VAD has been studied and through simulation the profile of different impellers and centrifugal pump of VAD have been selected in terms of Efficiency. In [4] recovery of myocardial with the help of ventricular assist device has been discussed. By increasing the recovery of myocardial recovery the need for heart transplantation can be postponed. In [5] it has been noted with the aid of CFD temporal distributions of the 
parameters for instance the velocity and the pressure in heart can be figured out, so by using this tool during cardiomyopathy simulation of left ventricle flow dynamics has been performed. From the results obtained from simulation, pathophysiology and progression of the disease can be determined. This paper reviews the current state of CFD for the purpose of the investigation of blood pumps, counting a useful basic reviews of the studies up to now, which should help designer of the device being built to pick the most proper technique; an outline of blood damage models and the challenges faced in actualizing them into CFD and cutting - edge gaps in knowledge [6].

\subsection{Problem Statement}

Major concerns regarding VADs are their hemodynamic performance and mechanical failure. Thrombosis and hemolysis play a crucial function inside the overall performance of the VADs. Thrombosis is the origination of a blood clot initiated by the way of the body's hemostatic mechanism to prevent bleeding.

Certain factors such as blood flow interruption, vascular wall abnormalities and alteration of the blood constitution can trigger the development of blood clots. [7] Formation of blood clots impacts the mechanism of the VAD through inflicting a drop-in flow and pumping power leading to the failure of the VAD if left untreated for a long time. Moreover, it may cause some fatal internal damage and is determined as one of the primary causes behind the demise of patients with the support of VAD and also reported to be responsible for up to $10 \%$ of device failure during the 2 years lengthy trial of HeartWare HVAD which is taken into consideration to be one of the most facilitated $3^{\text {rd }}$ generation heart pumps for BTT patients [1]. On the other hand, hemolysis is defined as the breakage of the membrane of Red Blood Cells (RBC) further leading to the injection of hemoglobin into the bloodstream. This injection may worsen the patient's condition by being the sole reason behind the deterioration of the kidney's functions resulting in multiple organ failure. Hemolysis mainly occurs due to the increased shear stress applied on the RBC. In case of a rotary blood pump high shear stress can be generated due to high rotational speed, therefore this is a major designing sphere in designing a centrifugal blood 
pump for the VAD. Both thrombosis and hemolysis are fatal problems and reasons of the device failure in most cases. Our aim is to overcome these problems to a large extent in our design.

\subsection{Methodology}

Computational Fluid Mechanics (CFD) is used as our most important analytical tool for this research. CFD is a department of fluid mechanics that solves issues related to fluid flow through the usage of high computational power. CFD is now broadly adopted in distinct engineering field due to its advantages over traditional techniques. These advantages encompass the potential to offer precise, visualized and comprehensive data that reduces time and production cost compared to experiment-based methods. In addition, using CFD solve complex flow problems can be solved that cannot be solved using analytical methods. CFD has been used as a computational tool in VAD design since the early ages of VAD developments. In the recent years usage of CFD has increased more along with the rising popularity of CF-VADs. CFD is widely utilized for calculating certain hydraulic parameters for instance, fluid forces on the rotor, Torque and Efficiency and furthermore the hemodynamic related traits and shear stress for the prediction of hemolysis. In our work ANSYS Fluent version 18.1 tool is used for the designing and simulation of the Bi-VAD.

\subsection{Overview of the Contents}

This thesis has been divided into 6 Chapters that comprises of the details of basic information of the VAD and CFD, required mathematical models and equations, basic structure and simulation system of the heart pump to be built, the results obtained upon simulations and a detailed summary of the entire research work done with future goals and scopes.

\section{Chapter 1 Introduction}

In this Chapter definition and importance of VADs are explained. Our motivations of choosing this research field, a brief literature review, problem statement and the methods required for solving these problems have also been talked about in this Chapter. 


\section{Chapter 2 Thesis Overview}

The system overview of this thesis, related mathematical models, simulation objective and procedures are presented briefly.

\section{Chapter 3 Mathematical Modeling for CFD analysis of the Pump}

In this Chapter all the required mathematical models and equations are discussed that are needed for successful analysis of fluid dynamics such as fluid model, flow model, turbulence model, blood damage model etc.

\section{Chapter 4 Designing and simulation of the VAD}

This Chapter contains different impeller designs that are going to be used for simulation. A few numbers of designs were made for observing the blood flow characteristics under different conditions. Short description of each design including design parameters, blade types, numbers etc. are presented in this Chapter. LVAD, RVAD and Bi-VAD main design aspects are also added separately.

This Chapter also discusses the simulation process of LVAD, RVAD and Bi-VAD designs. Different parameters such as inlet and outlet pressure difference, torque etc. are determined from the simulation. Simulations were performed under different conditions on several designs for LVAD. Results extracted from these simulations were compared and the best design was chosen for RVAD and Bi-VAD simulations.

SOLIDWORKS 2018 was used for the designing and ANSYS Fluent version 18.1 were use for the simulation purposes. 


\section{Chapter 5 Result}

The parameters gained from the simulation (pressure difference and torque) were used to calculate Head and Efficiency in this Chapter. The results were analyzed and presented in both tabular and graphical form for better understanding and evaluation. Furthermore, few contour profiles for instance, the pressure, velocity and shear stress profiles are shown for the visibility and hemodynamic evaluation of the designs of the impellers.

\section{Chapter 6 Conclusion}

This Chapter contains a brief summary of the results obtained from the research and focuses on the future development and scopes in the design and in this field.

In order to reach the destined point in the research work, several stages of work and methods were attempted. First and foremost stage was of the topic selection, i.e., the 3D problem the solver wants to work on. This thesis focused on the topic of eradicating heart failure problems through the 3D designing and later simulation of a Ventricular Assist Device (VAD) and then a prototype of the design can be constructed to be used in realistic situations when the supply of donor hearts is a major problem. Proceeding, after the topic has been selected, thorough background study and previous research works are brought into consideration for research purposes and for getting a brief idea on the problem to be worked upon. To meet the perfect outcome of fluid flow modeling in the thesis, Mathematical modeling of the pump will be done and utilized to bring forward the required information to solve the problem being worked upon to the solver. After this step, the impellers will be designed in SOLIDWORKS 2018 software and simulated with the support of the ANSYS Fluent version 18.1 software being used to get a visual demonstration of how the impellers work. Initially, the impellers will be designed for a single sided VAD (i.e., LVAD) and then simulated. With the obtained results from simulation, further calculations will be carried out and the resulting outcome will be analyzed and compared. The best design of the impeller will be selected after thorough analysis of the results with which the RVAD model and proceeding the Bi-VAD model will be simulated. Upon being simulated the results will be analyzed through the process of calculations and discussions and conclusion will 
be made on Efficiency of the final Bi-VAD model. Further conclusions will be made on the future scopes to work on research work by building a prototype of the best designed impeller being worked upon.

The block diagram of the thesis overview is shown below:

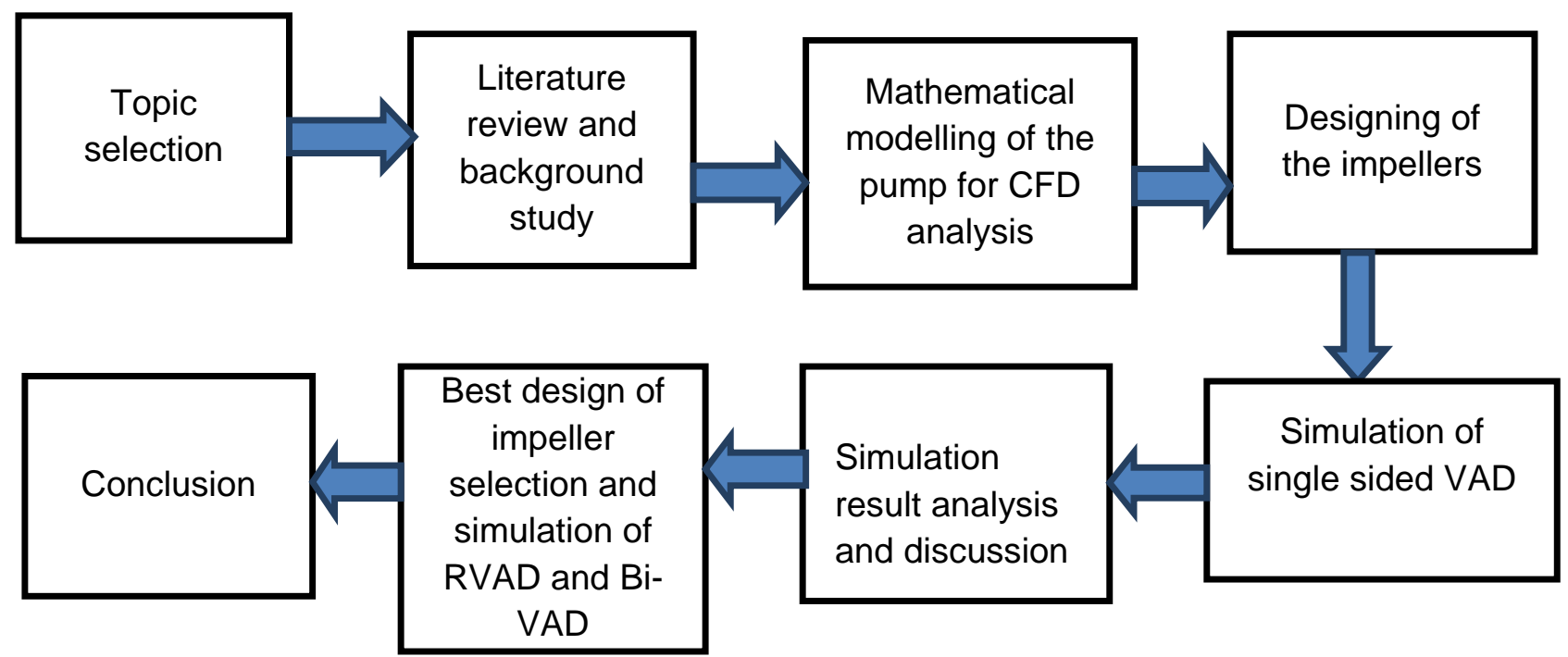

Figure1: Block Diagram of the thesis overview 


\section{Chapter 2}

\section{Overall System Overview}

One of the growing concerns nowadays is Heart Failure (HF), which may also be referred to as the Congestive Heart Failure (CHF). This situation arises when the heart fails to pump blood sufficiently to keep up with the blood flow in order to satisfy the body's needs. In this case, a patient may need an immediate heart transplant or effective pump of the blood circulatory system. Availability of donor is very much rare and hence throughout this time of heart failure, patient needs a temporary solution which will act more like an effective pump of blood circulatory system and replace the damaged heart of the patient. And this system is known as Ventricular Assist Device, VAD which is quite complicated structure. This thesis is mainly focusing on simulations of centrifugal pump of VAD.

The centrifugal pump of VAD consists of:

1. The Impeller

2. The Volute Casing

Therefore, the aim is to simulate VAD by Computational Fluid Dynamics studies under various criterions and choose the best impeller by which a centrifugal pump can be developed and it will circulate blood throughout the whole body in an effective manner. 


\section{Biventricular assist device (BiVAD)}

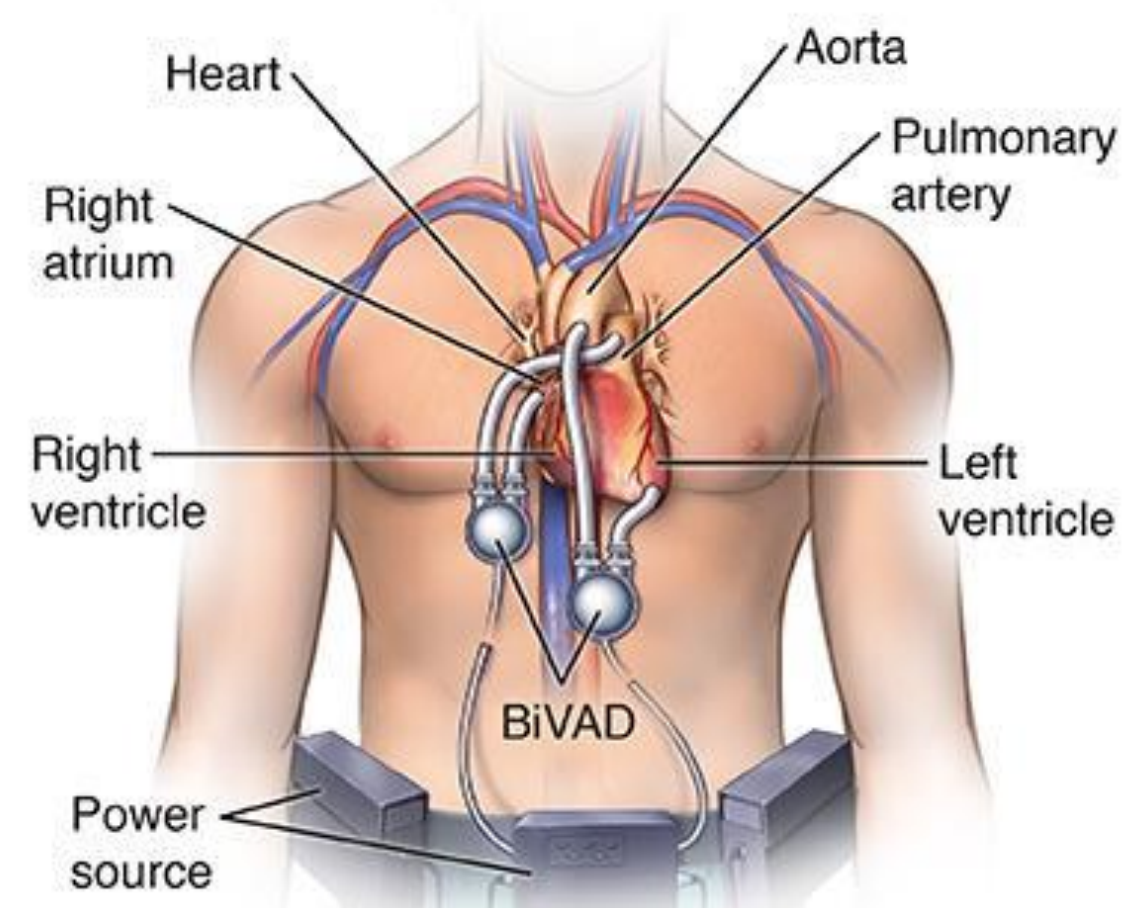

Figure 2.1: Visual demonstration of how a Bi-VAD is installed in a Human Heart.

\subsection{Design of Impeller}

Impeller is the rotating component within the heart pump to be built which can also be termed as the rotor of the pump. Impeller takes the blood from the respective part of heart and gives circulatory motion to the blood particles which will help blood to reach the required destination. Using SOLIDWORKS 2018 software, the impeller structures have been developed. These designs are made up based on various assumptions and criterions which is elaborately discussed on Chapter 4. Figure 2.1 is the main structure of impeller based on which a few variations have been made to create multiple designs and from them the best impeller has been selected through simulation process. 


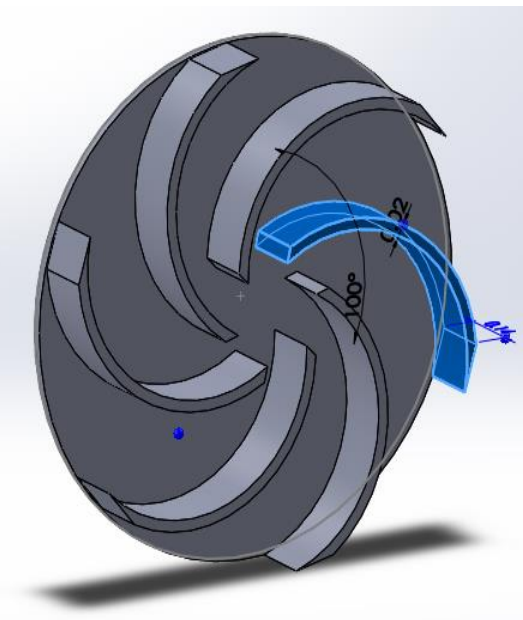

Figure 2.2: Structure of Impeller.

\subsection{Design of Volute casing}

A volute of a centrifugal pump is a curved channel that increments in vicinity as it draws closer to the discharge port. The volute is the casing that collects the blood being driven out by the impeller, by holding up and maintaining the speed at which the blood is flowing through to the diffuser. As fluid leaves the impeller it has high active kinetic energy and the volute coordinates this move through to the release. As the liquid goes along the volute, it is joined by increasingly more liquid leaving the impeller at the same time, as the cross-sectional region of the volute expands, the velocity is maintained. There are two parts in the volute casing and they are:

\section{Inlet Pipe}

II. Outlet Pipe

Inlet pipe takes the blood from the respective area where it is connected and passes it to the impeller. Impeller pumps the blood and passes it to outlet pipe. And outlet pipe dispenses the blood towards the specific point of affix. 


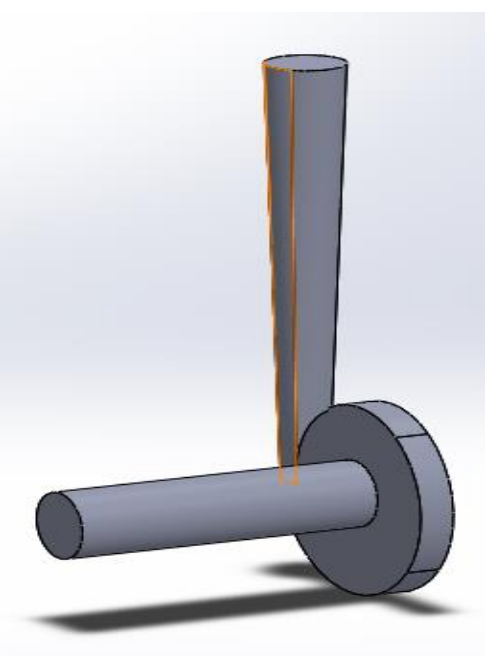

Figure 2.3: Volute casing with inlet and outlet

\subsection{Design of Bi-VAD}

Since the heart consists of two parts (the left and the right), two sets of centrifugal pumps are needed. In LVAD, the inlet pipe of centrifugal pump is connected with the left ventricle of the heart. From the left ventricle, the oxygenated and purified blood is gathered with the guidance of the inlet pipe. Afterwards blood is pumped by impeller and carried out by outlet pipe towards the aorta. And aorta will distribute the oxygen enriched blood to all parts of the body. In RVAD, the inlet pipe will be inserted at right ventricle. Following that right ventricle will provide deoxygenated blood through inlet pipe to impeller. Impeller will pump the blood and discharge it through outlet part in the direction of pulmonary artery. Pulmonary artery will forward the blood to the lungs for purification. 


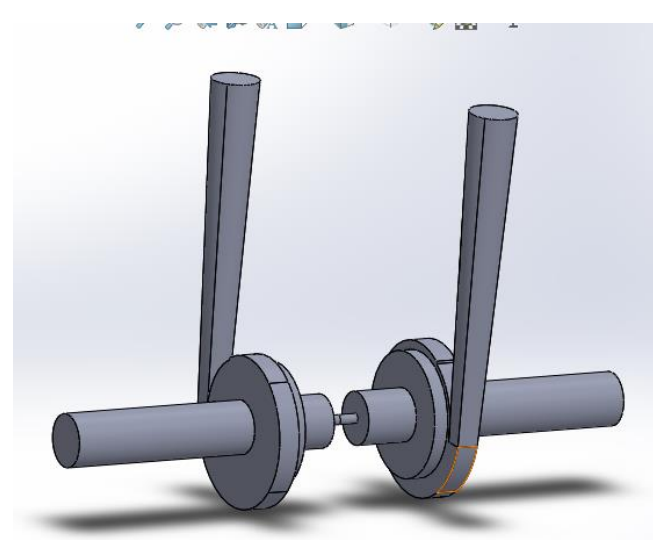

(a)

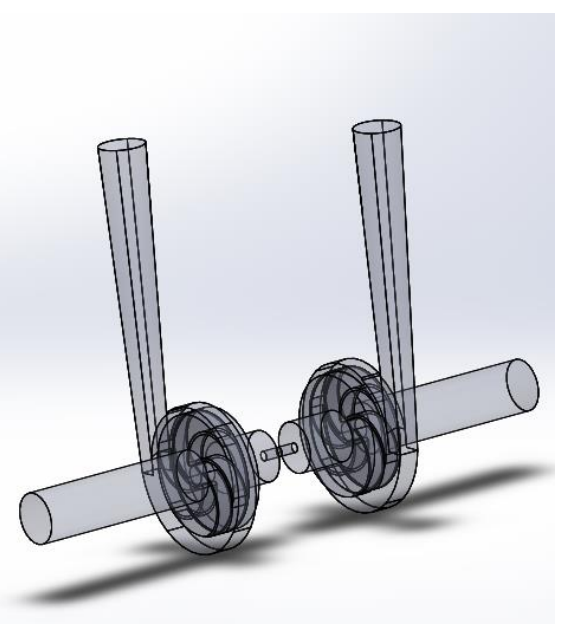

(b)

Figure 2.4: (a) Volute casing of the inlet and outlet of Bi-VAD model. (b) Impeller of pump with Volute casing.

\subsection{Simulation Process}

Designs of VAD structure is made under SOLIDWORKS and the simulation of computational fluid dynamics studies for VAD have been done by ANSYS Fluent version 18.1 software. The process of whole simulation is elaborately provided in Chapter4 and the results of simulation have been elaborately described in Chapter 5 of this paper. 


\section{Chapter 3}

\section{Mathematical Modeling for CFD analysis of the Pump}

\subsection{Introduction}

To meet the aim of getting a perfect result for fluid flow simulation in Computational Fluid Dynamics (CFD), one important criteria that needs to be met is the Mathematical Modeling of the fluid flow where certain parameters such as the fluid properties as well as the flow environments needs to be brought down to the problem solver with the aid of the fluid modeling, boundary conditions with a purpose to get a realistic idea of the working principle of the fluid modeling within the device to be built. In boundary conditions these parameters specified is applied to the inlet, outlet and surrounding walls of the impeller being designed to get a realistic view upon the fluid flow as it enters and leaves the surface being worked on. Working on CFD simulation that allows simulating the pulsatility of the blood flowing inwards, steady flow parameters are applied to the inlet and outlet of the impeller. Usually, parameters such as velocity or pressure are set depending on how the inlet and outlet is being classified and the values will be set depending on which side of the heart the solver is working on (i.e., LVAD or RVAD).

\subsection{Fluid Modeling}

In order to perform the CFD simulation for Bi-VAD one critical part of the analysis includes framing the properties and characteristics of the fluid (i.e., blood), such as viscosity, density, compressibility and others such as conductivity, vapor pressure depending on the requirements of the problem being worked on and so that the solver can get the perfect outcome of the problem being worked on and have higher expertise and understanding of the behavior of the flowing fluid to its external surroundings. Moreover, the values of the parameters being used are taken within a certain range due to its bulk property. Such as value of density is taken within a range of $1,040-1,060 \mathrm{~kg} / \mathrm{m}^{3}$ and viscosity within the range of $0.0030-0.0036$ $\mathrm{Pa}[7,8,9,10,11,12,13]$. Taking into consideration the fluid mechanics, blood is accounted for as 
a non - Newtonian compressible fluid that will be exerting a shear stress of $100 \mathrm{~s}^{-1}$ only when the blood will be showing off a Newtonian behavior $[14,15,16]$. However, most of the Computational Fluid (CF) pumps brings to light a shear stress that quite exceeds the range of shear stress rate specified for the Newtonian compressible fluid, so it is given the title of an incompressible fluid in the CFD simulations being performed and to avoid the amount of complexity in its composition blood is considered as a completely pure substance.

\subsection{Flow Modeling}

While analyzing the fluid dynamics, the fluid that is flowing through the rotating pump can be categorized into two types - one is the laminar flow and the other termed as the turbulent flow. Whether the fluid flow is laminar or turbulent may be decided with the aid of the Reynolds number and by further comparing it to the standard value of it.

\section{Reynolds Number}

The ratio for Reynolds number is given as follows:

$$
R e=\frac{\text { inertia force }}{\text { viscous force }}
$$

When the Reynolds number is low, then it indicates that the inertia forces in the fluid flow is also much smaller compared to the viscous forces present. Hence, the obstacles being faced throughout the fluid flow is overcome by the viscous forces and gets eliminated which leads to the flow being smooth without any kind of obstacles or disturbances. This type of flow will be categorized as the laminar flow of fluid.

However, if the Reynolds number is bigger than the standard value it indicates that the inertia forces are quite big and this will be leading to a much disturbed and full of obstacles fluid flow which will be categorized as the turbulent flow. But, due to the complexity in its nature this will 
be increasing the difficulties being faces while predicting due to the presence of eddies or vortices that leads to the random flow of fluid thus leading to a chaotic flow [1].

After brief analysis of the rotating pump the Reynolds number for the flow model can be written as [1] :

$$
R e=\frac{\rho \omega D^{2}}{\mu}
$$

Where,

$\operatorname{Re}=$ Reynolds number

$\rho=$ Density of the fluid

$\omega=$ Angular velocity of the impeller

$\mathrm{D}=$ Outer diameter of the impeller

$\mu=$ Fluid viscosity

Furthermore, for the rotating pump being used the transition from the laminar flow to the turbulence flow arises at around a value of $\operatorname{Re}=10^{5}[17]$.

\section{Navier Stokes equation}

The equations proposed by Claude - Louis Navier and George Gabriel Stokes are a set of governing equations being used in the mathematical modeling of the fluid flow to explain the motion of the viscous fluid that is flowing. The building blocks of this equation are the set of continuity-conservation equations, i.e., conservation of mass, conservation of momentum, conservation of energy and the conservation of the scalar quantities [1].

These equations are utilized in various fluid flow applications in the engineering field since it produces a brief description of the chaotic flow of fluid which can simply be termed as the turbulent flow of fluid but where it is lacking behind is that it requires very high computational power [1] or some other special approaches when being utilized in a more complex case of fluid flow. The main continuity equations can then be written as: 
1. Conservation of mass:

Taking a point $\mathrm{P}\left(x_{1}, x_{2}, x_{3}\right)$ to be the center of the volume element (the rotating pump through which the fluid will be flowing)

Let, the mass density at point $\mathrm{P}$ be $\rho\left(x_{1}, x_{2}, x_{3}\right)$.

Hence,

The total mass contained within $\mathrm{dv}$ is:

$$
M=\int \rho d v=\int \rho d x_{1} d x_{2} d x_{3}
$$

The surface area is termed as $\mathrm{dS}$ and $\dot{n}$ is the unit that is normal to that surface. In analysis:

$$
\begin{gathered}
\rho \vec{V} \cdot \overrightarrow{d S}=\rho \vec{V} \cdot \dot{n} d S \\
\sum \rho \vec{V} \cdot \overrightarrow{d S}=\oint \rho \vec{V} \cdot \overrightarrow{d S}=\oint \rho \vec{V} \cdot \dot{n} d S
\end{gathered}
$$

The rate at which mass, i.e.; the fluid flowing is entering or leaving through the surface of the pump $(\mathrm{dS})$ can be written as:

$$
\frac{d M}{d t}=\frac{d}{d t} \int \rho d V=-\oint \rho \vec{V} \cdot \dot{n} d S
$$

For a fixed surface, the total time derivative inside the volume integral can be written as:

$$
\int \frac{\partial \rho}{\partial t} d V=-\oint \rho \vec{V} \cdot \dot{n} d S
$$

Applying Gauss's theorem the achieved equation is shown as follows:

$$
\begin{gathered}
-\oint \rho \vec{V} \cdot \dot{n} d S=\int(\vec{V} \cdot \rho \vec{V}) d V \\
\int\left[\frac{\delta \rho}{\delta t}+\vec{\nabla} \cdot(\rho \vec{V})\right] d V=0
\end{gathered}
$$

This expression must hold on for every arbitrary shaped volume, the only way that it can be satisfied is if the integrand vanishes identically or [18] : 
$\frac{\partial \rho}{\partial t}=-\nabla \cdot(\rho U)$

This is called the continuity equation for the conservation of mass.

2. Conservation of momentum [1] :

$$
\rho g-\nabla \rho+\nabla \cdot \tau_{i j}=\rho \frac{d U}{d t}
$$

3. Conservation of energy [1] :

$$
\rho \frac{d h}{d t}=\frac{d P}{d t}+\nabla \cdot(k \nabla T)+\varphi
$$

4. Conservation of scalar quantities [1] :

$$
\frac{\partial(\rho \varphi)}{\partial t}+\nabla(\rho \varphi V)=\frac{d P}{d t}+\nabla \cdot(k \nabla T)+\varphi
$$

Two methods are implemented to resolve the Navier Stokes equation for the simulation of the fluid flow mechanism. One is the Direct Numerical Solution (DNS) which is taken to be the most accurate flow simulation model. This method directly solves the Navier Stokes equations without using any kind of approximations or averaging parameters other than numerical discretizations $[19,20]$. However, this method is not the most ideal method for calculation since it requires high computational power during its usage to solve the complicated Navier Stokes equations. The alternative method termed as the Large Eddy Simulation (LES) method which when implemented sets its focal point on solving the large scale eddy or vortices that is more frequent when the fluid flow is turbulent [1] . Moreover, this method is cheaper than the DNS method and given the highest priority when solving cases for complicated geometry and the chaotic turbulence fluid flow [20]. 
However, in large scale engineering applications, the high accuracy methods; i.e., DNS and LES methods consumes and wastes a lot of resources. Hence, the Reynolds Averaged method is considered as a more reliable choice for the fluid simulation since it produces much less information however offers sufficient approximation values that could then be implemented [1].

\section{Reynolds Averaged equation}

This method considers the time averaging of all the variables with a purpose to eliminate the fluctuations inside the flow properties.

The Reynolds decomposition technique is used for the purpose of the calculation taking into account that any flow property, $(\varphi)$ can be decomposed and written as the summation of its time averaged value, $(\bar{\varphi})$ and the fluctuations that are present, $(\grave{\varphi})$ [19]:

$$
\varphi\left(x_{i}, t\right)=\bar{\varphi}\left(x_{i}\right)+\grave{\varphi}\left(x_{i}, t\right)
$$

Along with the Reynolds decomposition for the flow property, the instantaneous velocity (u) of the fluid flowing can also be decomposed and written as the summation of the mean velocity $(U)$ and the fluctuating part (ù) as shown below [1]:

$$
\begin{aligned}
& u_{i}\left(x_{i}, t\right)=U_{i}\left(x_{i}\right)+u_{i}\left(x_{i}, t\right) \\
& U_{i}\left(x_{i}\right)=\lim _{T \rightarrow \infty} \frac{1}{T} \int_{0}^{T} u_{i}\left(x_{i}, t\right) d t
\end{aligned}
$$

Since it is known,

$$
u_{i}\left(x_{i}, t\right)=U_{i}\left(x_{i}\right)+u_{i}\left(x_{i}, t\right)
$$

Hence, $U_{i}\left(x_{i}\right)$ is called the average of $u_{i}\left(x_{i}, t\right)$.

Therefore, it can be shown that [1] : 


$$
\begin{gathered}
U_{i}\left(x_{i}\right)=\lim _{T \rightarrow \infty} \frac{1}{T} \int_{0}^{T}\left(U_{i}\left(x_{i}\right)+\grave{u}_{\imath}\left(x_{i}, t\right)\right) d t \\
=\lim _{T \rightarrow \infty} \frac{1}{T} \int_{0}^{T}\left(U_{i}\left(x_{i}\right)\right) d t+\lim _{T \rightarrow \infty} \frac{1}{T} \int_{0}^{T} \grave{u}_{\imath}\left(x_{i}, t\right) d t \\
\left.=\left(U_{i}\right)+0 \text { (i.e.; the fluctuating part is considered to be } 0\right)
\end{gathered}
$$

Since, the limits on integration on $U_{i}\left(x_{i}\right)$ is constant hence [1],

$$
\frac{\overline{\partial U_{i}}}{\partial x_{i}}=\frac{\partial \overline{U_{i}}}{\partial x_{i}}
$$

Consequently, applying the time averaged velocity components to the Navier Stokes continuity equations; conservation of mass and conservation of momentum with the idea of an incompressible and steady property flow the Reynolds Averaged Navier Stokes equation (RANS) can be represented as [1] :

$$
\rho \frac{\partial U_{i}}{\partial t}+\rho U_{j} \frac{\partial U_{i}}{\partial x_{j}}=-\frac{\partial P}{\partial x_{i}}+\frac{\partial}{\partial x_{i}}\left(\tau_{i j}-\rho \overline{\grave{u}_{j}} \bar{u}_{\imath}\right)
$$

Where,

$\tau_{i j}=$ viscous stress

The term $\rho \overline{\hat{u}_{j} \bar{u}_{\imath}}$ has a constantly changing contribution to the non linear acceleration terms and acts as a stress; therefore termed as the Reynolds Stress Tensor [21]. Introducing this term introduces 6 unknown quantities alongside the pressure and the 3 velocity components thus altogether a total of 10 unknowns are present in the equation. However, the available equations are the Navier Stokes continuity equation - conservation of mass and the 3 components of the Reynolds Averaged Navier Stokes equation (RANS) building up as 4 equations in total. Hence, proving that in comparison to the number of equations there is more number of unknowns therefore it is not unusable or a closed system [22]. As an alternative technique, approximations are embedded into the Reynolds stress term and the modified equation is termed as the Turbulence Model. 


\subsection{Turbulence Model}

Amongst the numerous categories of turbulence modeling, the eddy viscosity model is one of the most commonly used model for CFD applications which takes into account that the chaotic, turbulent flow includes small eddies or vortices and furthermore the statement that the Reynolds stress term is proportional to the mean velocity gradients. At that point the stress of an interested time is the outcome of the velocity everywhere from the past point in time to that specific point $[21,23]$. Due to this the transport equations needs to implant into itself not only the nonlocal but as well as the flow history effects of the eddy viscosity $\left(\mu_{t}\right)$. Consequently, the turbulence kinetic energy $(\mathrm{k})$ was selected for the velocity scale in the energy equation [22].

The eddy viscosity can be decomposed and written as follows [1] :

$$
\mu_{e f f}=\mu+\mu_{t}
$$

Where,

$\mu_{e f f}=$ Local effective viscosity

$\mu=$ Local molecular viscosity

$\mu_{t}=$ Local turbulent viscosity

The instantaneous turbulence kinetic energy can be decomposed and shown as the summation of the mean value and the fluctuating part as follows [1] :

$$
k(t)=\overline{k(t)}+k \grave{(t)}
$$

The mean kinetic energy $\overline{k(t)}$ can be represented as:

$$
\overline{k(t)}=\frac{1}{2}\left(\overline{u^{2}}+\overline{v^{2}}+\overline{w^{2}}\right)
$$

Therefore, the relationship of the turbulence kinetic energy $(\mathrm{k})$ to the velocity scale which tells about how the mean flow feeds the kinetic energy into turbulence is shown as [1] : 


$$
k=\frac{1}{2} \overline{\grave{u_{\imath}}}=\frac{1}{2}\left(\overline{u^{2}}+\overline{v^{2}}+\overline{\bar{w}^{2}}\right)
$$

In order to build the turbulence energy equation, the Boussinesq eddy viscosity assumption was implanted which states that the Reynolds stress tensor represented by $\tau_{i j}$ is proportionally associated with the strain rate tensor represented by $\sigma_{i j}$ as follows [1] :

$$
\tau_{i j}=2 \mu_{t} \sigma_{i j}+\frac{2}{3} \delta_{i j} \rho k
$$

Since, an assumption of compressible flow is made so for that the value of the strain rate tensor is taken as, $\sigma_{i j}=0$ [1] and hence by bringing forth modifications in the equation shown for Reynolds stress tensor, the modified equation can be shown as [1] :

$$
\tau_{i j}=-2 k
$$

According to all the assumption such as the fluid is incompressible that are embedded, the equation of the turbulence energy and the eddy viscosity by the aid of the Navier Stokes continuity equation - conservation of mass and conservation of momentum can be written as follows $[1,22]$ :

$$
\frac{\partial \rho k}{\partial t}+U_{j} \frac{\partial \rho k}{\partial x_{j}}=\rho \tau_{i j} \frac{\partial U_{i}}{\partial x_{j}}-\rho \varepsilon+\frac{\partial}{\partial x_{j}}\left[\left(\mu+\frac{\mu_{t}}{\sigma_{k}}\right) \frac{\partial k}{\partial x_{j}}\right]
$$

Where,

$\varepsilon=$ Eddy dissipation rate

$\sigma_{k}=$ closure coefficient $\left(\sigma_{\mathrm{k}}=1.0\right)$

The eddy dissipation rate parameter $(\varepsilon)$ which by definition is the rate of dissipation of the turbulence kinetic energy $(\mathrm{k})$ can be represented in mathematical form as follows [1] : 


$$
\varepsilon=\frac{k^{\frac{3}{2}}}{l}
$$

Where,

$l=$ turbulence length scale

However, 2 unknown parameters are introduced in the turbulence kinetic energy equation, i.e., $\varepsilon$ and $l$ which can be approximated by methods included with the turbulence model. These methods can be labeled into sorts with the aid of the number of partial differential equations that it includes with itself. These methods are especially categorized and termed as the Zero equation model, One - equation model and the Two - Equation model [1].

\section{Zero - Equation Model}

This model is termed as such since it does not embed into itself any extra partial differential equations rather the eddy viscosity is calculated from the algebraic equation fabricated from the turbulence length scale and the turbulent viscosity scale as shown below [1] :

$$
\mu_{t}=\rho f_{\mu} U_{t} l
$$

Where,

$\mu_{t}=$ eddy viscosity

$f_{\mu}=$ proportionality constant

$U_{t}=$ turbulent viscosity scale

$l=$ turbulence length scale

The turbulence length scale can further be written as follows for simplified calculation purpose [1] :

$$
l=\frac{V_{D}^{1 / 3}}{7}
$$


Where,

$V_{D}=$ volume of fluid domain

When this Zero - equation model is substituted in the transport equation, i.e., RANS equation to solve the unknown parameters ( $\varepsilon$ and $l$ ), although it requires very less computational power but the part where it is lacking and it is not chosen over the other model is that it does not perfectly provide the building block of the system and therefore cannot give the best outcome when used in general fluid flow problems [1].

\section{One - Equation Model}

Similarly to the previously explained Zero - Equation model, this model is named as such because of the fact that it introduces one partial differential equation. The One - Equation model is also named as the Prandtl's model. Similar to the Zero - Equation model, this model approximates the unknowns and solves the transport equation through the relationship between the eddy viscosity shown as a function of the turbulence kinetic energy, the turbulence length scale and the newly introduced parameter for the approximation of the unknown parameters in the transport equation termed as closure coefficient as follows [1]:

$$
\varepsilon=\frac{C_{D} k^{3 / 2}}{l}
$$

Where,

$\varepsilon=$ turbulence dissipation which is a function of the turbulence kinetic energy

$l=$ turbulence length scale

$C_{D}=$ Closure co - efficient

$k=$ turbulence kinetic energy 
Likewise, to the Zero - Equation model the eddy viscosity in the One - Equation model can be represented in a certain way as shown [1] :

$$
\mu_{t}=k^{1 / 2} l
$$

Substituting the above equation for the turbulence dissipation in the equation for eddy viscosity to further simplify [1] :

$$
l=\frac{C_{D} k^{3 / 2}}{\varepsilon}
$$

Hence [1], :

$$
\begin{aligned}
\mu_{t} & =k^{1 / 2}\left(\frac{C_{D} k^{\frac{3}{2}}}{\varepsilon}\right) \\
& =k^{2} \frac{C_{D}}{\varepsilon}
\end{aligned}
$$

Since, the Zero - Equation model cannot be used due to certain limitations hence the One Equation model is used to solve the unknown parameters in the transport equation, i.e., RANS equation found previously. Substituting the One - Equation model in the RANS equation of the turbulence energy and the eddy dissipation a modified mathematical equation for the turbulence energy is achieved [1] :

Substituting the modified equation is as follows:

$$
\varepsilon=\frac{C_{D} k^{3 / 2}}{l}
$$

The new, modified equation is shown as:

$$
\begin{gathered}
\frac{\partial \rho k}{\partial t}+U_{j} \frac{\partial \rho k}{\partial x_{j}}=\rho \tau_{i j} \frac{\partial U_{i}}{\partial x_{j}}-\rho \varepsilon+\frac{\partial}{\partial x_{j}}\left[\left(\mu+\frac{\mu_{t}}{\sigma_{k}}\right) \frac{\partial k}{\partial x_{j}}\right] \\
=\frac{\partial \rho k}{\partial t}+U_{j} \frac{\partial \rho k}{\partial x_{j}}=\rho \tau_{i j} \frac{\partial U_{i}}{\partial x_{j}}-\rho \frac{C_{D} k^{3 / 2}}{l}+\frac{\partial}{\partial x_{j}}\left[\left(\mu+\frac{\mu_{t}}{\sigma_{k}}\right) \frac{\partial k}{\partial x_{j}}\right]
\end{gathered}
$$


Where, the value of the parameter $\sigma_{k}$ (closure co-efficient) is taken to be 1.0 [1]. This is the modified mathematical equation of the turbulence kinetic energy.

\section{Two - Equation Model}

Like its name suggests it introduces 2 partial differential equations. This model mainly derives the transport equation, i.e., RANS equation through the aid of 2 types of scalar parameters, one of which relies on the turbulence kinetic energy and the other turbulence length scale or any other equivalent parameters. In this case, the second parameters are the turbulence eddy dissipation $(\varepsilon)$ and specific eddy dissipation $(\omega)$. The two basic models used under the Two Equation model are the $\mathrm{k}-\varepsilon$ and the $\mathrm{k}-\omega$ model.

\section{$\mathbf{k}-\varepsilon$ Model}

This model is one of the most broadly used model for performing CFD simulations and various other field and is even being utilized in the Computational Fluid (CF) heart pump simulations [1]. As its name suggests the parameters that this model uses to solve the transport equation are the turbulence kinetic energy and the turbulence eddy dissipation. For solving the relationship that it requires is the relationship of these two parameters to the turbulent viscosity as shown below [1]:

$$
\mu_{t}=\rho C_{\mu} \frac{k^{2}}{\varepsilon}
$$

By expressing this in terms of the turbulence kinetic energy in the transport equation [1] :

$$
\frac{\partial \rho k}{\partial t}+U_{j} \frac{\partial \rho k}{\partial x_{j}}=\rho \tau_{i j} \frac{\partial U_{i}}{\partial x_{j}}-\rho \varepsilon+\frac{\partial}{\partial x_{j}}\left[\left(\mu+\frac{\mu_{t}}{\sigma_{k}}\right) \frac{\partial k}{\partial x_{j}}\right]
$$

The transport equation in terms of the turbulence kinetic energy can further be expressed in terms of the eddy dissipation rate as follows [1]: 


$$
\frac{\partial \rho \varepsilon}{\partial t}+U_{j} \frac{\partial \rho \varepsilon}{\partial x_{j}}=C_{\varepsilon 1} \frac{\varepsilon}{k} \rho \tau_{i j} \frac{\partial U_{i}}{\partial x_{j}}-C_{\varepsilon 1} \rho \frac{\varepsilon^{2}}{k}+\frac{\partial}{\partial x_{j}}\left[\left(\mu+\frac{\mu_{t}}{\sigma_{k}}\right) \frac{\partial \varepsilon}{\partial x_{j}}\right]
$$

The values of the closure coefficients are given as follows [22]:

$C_{\varepsilon 1}=1.44$

$C_{\varepsilon 1}=1.92$

$C_{\mu}=0.09$

$\sigma_{k}=1.0$

$\sigma_{\varepsilon}=1.3$

Although this model is computationally very cheap and easy to be implemented in Computational Fluid (CF) heart pump simulations but even after this the drawbacks of this model is its insensitivity to the adverse pressure gradient and robust flow in addition to the low performance in analyzing the viscous sub - layer [24,25,26]. Due to all these reasons this model should be avoided for the implementation in cases where the pressure gradient changes rapidly but it is the most appropriate model for comparing and implementing in different cases and geometries [1].

\section{$\mathbf{k}-\omega$ Model}

Similarly to the $\mathrm{k}-\varepsilon$ Model this model also attends in solving turbulence equation with the aid of two partial differential equations and solves the unknown parameters in the transport equation, i.e., RANS equation by two scalar parameters: the turbulence kinetic energy $(\mathrm{k})$ and the other is the newly introduced parameter; specific rate of dissipation or turbulent frequency represented by $\omega$ which is defined as the rate of dissipation on energy in volume and time. The relationship of $\mathrm{k}$ and $\omega$ with the eddy viscosity can be further represented as [1] :

$$
\mu_{t}=\rho \frac{k}{\omega}
$$


A relationship may be developed in between the turbulence eddy dissipation and the specific eddy dissipation as follows [1] :

$$
\varepsilon=\beta k \omega
$$

Hence, the turbulence kinetic energy can be represented as [1] :

$$
\frac{\partial \rho k}{\partial t}+U_{j} \frac{\partial \rho k}{\partial x_{j}}=\rho \tau_{i j} \frac{\partial U_{i}}{\partial x_{j}}-\rho \beta k \omega+\frac{\partial}{\partial x_{j}}\left[\left(\mu+\frac{\mu_{t}}{\sigma_{k}}\right) \frac{\partial k}{\partial x_{j}}\right]
$$

This turbulence kinetic energy equation can be represented in terms of the specific eddy dissipation as follows [1] :

$$
\frac{\partial \rho \omega}{\partial t}+U_{j} \frac{\partial \rho \omega}{\partial x_{j}}=\alpha \frac{\omega}{k} \rho \tau_{i j} \frac{\partial U_{i}}{\partial x_{j}}-\rho \beta \omega^{2}+\frac{\partial}{\partial x_{j}}\left[\left(\mu+\frac{\mu_{t}}{\sigma_{k}}\right) \frac{\partial \omega}{\partial x_{j}}\right]
$$

The values of the constants are given as follows [1] :

$\beta=0.09$

$\alpha=5 / 9$

$\beta=0.075$

$\sigma_{k}=2.0$

$\sigma_{\omega}=2.0$

This model also has some advantages and drawbacks. The advantages consists of that it perfectly brings out the complicated boundary layer flows beneath an adverse pressure gradient. However its main drawback includes that the implementation gets complicated and computational cost increases more than the other model since it will be requiring a fine mesh resolution near the wall of the device as a way to be worked on and moreover the prediction of the separation seems out to be anticipated [1]. 


\section{SST k $-\omega$ Model}

This model is the carried out mixture of the two preceding models, i.e., $\mathrm{k}-\varepsilon$ and the $\mathrm{k}-\omega$ model. The $\mathrm{k}-\omega$ model is implemented in the viscous layer and the $\mathrm{k}-\varepsilon$ model is implemented in the stream layer. Although both these models is observed to provide with perfect results in the VAD simulation but the main underlying problem is that there isn't any proper investigation of determining the level of accuracy between these 2 models. It has been suggested previously by Song et al. [27], that the flow visualization plot of the $\mathrm{k}-\omega$ model within the area near the wall has a better relation with the particle velocimetry results compared to the $\mathrm{k}-\varepsilon$ model but later it has also been suggested by Throckmorton et al. [28], that the bulk parameters from the fluid flow system has a better connection and was closely similar to that of the experimental results achieved more than the $\mathrm{k}-\omega$ model depending on the miniature axial pump verification data.

\subsection{Blood Damage Model}

Blood damage is one of the most vital components in the blood flow model that is to be considered at the same time as designing a blood pump. However, it is one of the most difficult factors to be taken into consideration due to the massive biological diversity and the chemical properties that largely are laid low with the small changes brought in the test results and also handling during blood preparation for which the test results are almost non - reproducible [1]. The very first blood damage model developed by Giersiepen et al. [29], was developed on the premise of the empirical statistics provided by Wurzinger et al. [30] which showed that the blood damage model genuinely turned out to be only dependent on the shear stress and the exposure time as shown below [1] :

$$
D=\frac{d H b}{H b}=C \tau^{\alpha} \tau^{\beta}
$$

Where,

$D=$ damage fraction

$\frac{d H b}{H b}=$ ratio of plasma free hemoglobin to total hemoglobin 
$\tau=$ sheer stress

$t=$ exposure time

$C, \alpha$ and $\beta$ are depicted as constants with values of $3.65 \times 10^{-5}$, however, these values were quite overestimated and was replaced quite a lot of times to be implemented in different cases but as suggested by Heuser et al [29] the values were then replaced and then taken to be: $C=$ $1.8 \times 10^{-8}, \alpha=1.991, \beta=0.765$ for $t<0.6 s$ and $\tau<700 P a$. However even after this, investigation performed on centrifugal blood pumps by $\mathrm{Wu}$ et al [13] proved that even after changing the values, it yielded no big difference and each could generate and estimate the value of hemolysis yielded via the blood pump. The implementation of the blood damage model generated from the devices can further be divided into either the Lagrangian co-ordinates type or the Eulerian co-ordinates type [1].

\section{Lagrangian Method}

This method mainly relies upon calculating the shear stress rate that is being exerted on every individual blood cell as the blood is flowing through the device. The blood damage approximation is made in this approach via the "particle tracking technique" so as to be liberating blood particles at the inlet side of the pump and will be carrying out the integrating process of the damage function alongside that pathway and then the total blood damage will be calculated from the average of the damage that will be caused to the blood particles as it will be flowing through the device [1]. According to an investigation carried out a total of 1,100 particles were considered to be enough for the calculation of the blood pump but this estimated number can also vary with the device size being used [31]. According to the previously found equation of the blood damage model, the total damage alongside the pathway of the device covering the whole direction from the inlet to the outlet can be calculated as follows [1] :

$$
D=\frac{d H b}{H b}=C \tau^{\alpha} \tau^{\beta}
$$

Hence,

Total damage, $D=\int_{\text {inlet }}^{\text {outlet }} C \cdot d t^{\alpha} \cdot \tau^{\beta}=\sum_{i=1}^{N} C \cdot \Delta t_{i}^{\alpha} \cdot \tau_{i}^{\beta}$ 
Where,

$\mathrm{N}=$ number of observed points along the pathway of the device.

$t_{i}=$ Time that the particle took to start from the starting point and reach the $i^{\text {th }}$ set destination point.

However, Grigioni et al. advised that the summation of the individual damage being prompted to the individual cells as it passes through the device could not flawlessly mirror the image of a cell with sub - lethal damage which could further be damaged under shear stress $[32,33]$. Consequently, the following equation is used which could include the damage contribution within the summation as the erythrocyte moves along the pathway of the device [1] :

$$
D=\sum_{i=1}^{N} \operatorname{Ca}\left[\sum_{j=1}^{i} \tau\left(t_{j}\right)^{b / a} \Delta t\right]^{a-1} \tau\left(t_{j}\right)^{b / a} \Delta t
$$

However, further research and investigation was carried out on centrifugal and axial types of blood pump to see the accuracy for both the equations and it was seen that [1] :

$$
D=\int_{\text {inlet }}^{\text {outlet }} C \cdot d t^{\alpha} \cdot \tau^{\beta}=\sum_{i=1}^{N} C \cdot \Delta t_{i}^{\alpha} \cdot \tau_{i}^{\beta}
$$

This equation yielded less than $10 \%$ error in calculating the hemolysis of the blood pump whereas the second equation overestimated the hemolysis of the blood pump [1].

$$
D=\sum_{i=1}^{N} C a\left[\sum_{j=1}^{i} \tau\left(t_{j}\right)^{b / a} \Delta t\right]^{a-1} \tau\left(t_{j}\right)^{b / a} \Delta t
$$

Hence, this method could not give accurate outcomes in calculating and verifying the hemolysis of the blood pump at different values of shear stress rate [1]. Moreover, another estimation model was taken into consideration which will be taking the shear loading history into account. For this, it going to be dividing the pathway of the device being utilized in small sections so that 
the value of the shear stress rate can then taken into consideration to be steady at the small sections [1]. The damage index, D along this pathway can then be evaluated as [1] :

$$
D(t+\Delta t)=C \cdot\left(t_{e f f}+\Delta t\right)^{\alpha} \cdot \tau(t+\Delta t)^{\beta}
$$

Where,

$t_{\text {eff }}=$ virtual time steps which can be calculated as follows [1] :

$$
t_{\text {eff }}=\left(\frac{D(t)}{\operatorname{C.t}(t+\Delta t)^{\beta}}\right)^{\alpha}
$$

As suggested the values of the constants by Heuser et al, the values were then to be: $C=$ $1.8 \times 10^{-8}, \alpha=1.991, \beta=0.765$ [1] but for these values the output result was much less than that compared to the experimentally derived values. The accuracy of this approach specifically is dependent upon the number of blood particles which can be released at the inlet of the device. Consequently, this method will be requiring a huge amount of computational power seeing that a lot of particles are getting tracked alongside the pathway of the device [1].

\section{Eulerian Method}

Unlike the Lagrangian Method, the Eulerian Method mainly calculates the shear stress rate depending on the blood damage that is being caused over an entire domain at a specific point in time hence integrating it the Eulerian frame about the whole domain [1]. The accuracy of the blood damage calculation via the Eulerian method entirely depends on the mesh structure. Furthermore, this method requires less computational power compared to the Lagrangian method since this method does not need to track the particle through the pathway of the device. Based on the blood damage model structured based on the shear stress and exposure time [1]:

$$
D=\frac{d H b}{H b}=C \tau^{\alpha} \tau^{\beta}
$$


An hemolysis estimation technique fashioned from the experimental outcomes of blood exposed to various shear stress and exposure time suggested by Garon and Farinas with original regular set featuring the linear damage $\left(D_{I}\right)$ is as follows [34] :

$$
D_{I}=D^{1 / 0.785}=\left(3.62 \times 10^{-7} \frac{1}{0.785} \tau^{\frac{2.416}{0.785} t}\right.
$$

To lessen the complexity and further simplify by the time derivative to cast off the time dependency with the aid of an incompressible fluid assumption the equation for the average linear damage $\left(\overline{D_{I}}\right)$ is received as follows [1] :

$$
\overline{D_{I}}=\frac{1}{Q} \int \sigma d V
$$

Where,

$V=$ finite volume

$\sigma=$ sheer stress

Hence, in the Eulerian frame the damage function (D) generated from the device is proven to be as follows [1] :

$$
D=\left(\underline{D I}^{0.785}\right.
$$

This relation further gives an idea about the relationship between the damage function represented as D and the normalized index of hemolysis termed as NIH which is acquired only if there is a certain specific ratio in between the plasma free hemoglobin and the total hemoglobin, i.e., if the plasma free hemoglobin is smaller in amount than the total hemoglobin so then the equation for the average linear damage can further be simplified as follows which is used for various types of pump developments $[10,35]$ :

$$
N I H=H b \times D \times 100
$$


By using this method to calculate and evaluate the blood damage model is quite efficient since it will not need the aid of any computational load while the simulation is ongoing. However, where it is lacking is that it cannot provide the solver with the exact position of the blood damage since it only allocates a global index applicable across the entire domain to the solver [1].

Moreover, an additional hemolysis estimation method was proposed by Taskin et al. which brought to light plasma free hemoglobin represented by $H b$ and its mathematical equation is shown as follows [36] :

$$
\Delta H b=\Delta H b^{1 / \alpha}
$$

Thus, the transport equation in scalar form is obtained as follows [1] :

$$
\frac{d(\Delta H b)}{d t}+v \rho \nabla(\Delta H b)=S
$$

Where, $\mathrm{S}$ is the source term that is represented as follows [1] :

$$
S=\rho\left(H b \cdot C \tau^{\beta}\right)^{\frac{1}{\alpha}}
$$

As this is a new hemolysis model, for that a new set of values for the constants were replaced and introduced such as: $C=1.21 \times 10^{-5}, \alpha=0.747, \beta=2.004$ [1]. The damage index can then be calculated from the mathematical equation of the mass weighted average of $\mathrm{Hb}$ at the outlet of the device divided by the value of $\mathrm{Hb}$ [1]. The main advantage that this model has is that it provides the required information on the regions with high concentration of hemolysis which indeed is a very crucial information to be utilized during the designing procedure of the devices that are to be built. Although, this newly introduced hemolysis model when being calculated from the mathematical equations by substituting the values of the constants shows a sign of overestimation in the results [37]. Hence, to bring forward improvements in the prediction of the hemolysis outcomes for both the centrifugal as well as the axial pumps a new set of values of 
constants were further introduced for the calculation purpose as such:C $=1.745 \times 10^{-6}, \alpha=$ $1.963, \beta=0.7762[1]$. 


\section{Chapter 4}

\section{Design and Simulation of Ventricular Assist Devices (VADs)}

This Chapter explains the design concept with inclusion of required standards and criteria in order to conduct optimized CFD simulations of LVAD, RVAD and consequently Bi-VAD to speculate an understanding of the hydrodynamic and hemodynamic performances of the established designs. The designs being constructed in SOLIDWORKS 2018 are imported to ANSYS Fluent 18.1 software to conduct further simulations required to calculate and compare relevant parameters.

\subsection{Overview}

The development of VAD must be focusing not only on the hydraulic performance of the pump that is optimizing its operating Efficiency but also its hemocompatibility. As discussed in Chapter 2, the human circulatory system is complex and any artificial system associated with it, if not handled with proper care and provided precise realistic dimensions, can lead to major complexities such as thrombosis resulting in demise of patients.

The designs aim to operate as supporting pumps to the heart with LVAD for left ventricle and similarly RVAD for right ventricle and eventually Bi-VAD for both. Hereby, the ultimate goal of the contrived designs is to gain maximum Efficiency with minimum chances of hemodynamic casualty keeping the Head pressure within the desired range.

Furthermore, the designs are to be consequently placed within the pericardial cavity or abdomen of the patients and hence the size of these pumps must be small enough to successfully get accommodated there. Also, an optimum strength and a dependable mechanism will ensure increased working life and sustainability of the pump. 


\subsection{Design and Construction}

\subsubsection{VAD Design Background}

The initial stage of the designs of the impeller has been done by SOLIDWORKS 2018 software since it is easy to learn and operate and is a well-recognized tool for $3 \mathrm{D}$ designing. It is therefore a suitable option for the construction of the centrifugal pump of the VAD, through which pumped blood will be carried throughout the whole body. These designs must be very precise and error free to ensure minimum chances of instrumental failure. The dimensions are provided to the centrifugal pump in such a way so that it easily can accommodate in the chest area of human being.

\subsubsection{VAD Design Structure}

Primarily, six impellers are designed with variations in the wrap angle and blade number. Wrap angle can be defined as the angle in between the tangent lines at the leading and the trailing edges of the blade. If wrap angle increases the parameters being calculated, Head pressure and Efficiency also increases. However, the wrap angles are kept to an optimum value. Because, from studies [38] it has been found that optimum values of wrap angle such as $90^{\circ}, 100^{\circ}, 110^{\circ}$ and $120^{\circ}$ gives best Efficiency and increased Head pressure. So, wrap angles are considered among these values between 6 and 5 blades to make a comparative study which will provide the best Head and Efficiency. The objective of choosing 5 blades and 6 blades because in a research [39] it has been found that 5 blades and 6 blades gives the good amount of Efficiency with the required range of Head with less hemolysis while it has been stated that 4 blades and 7 blades are not giving the desired conditions that is required from the pump. For these reasons other number of blades is not taken in the light of concern.

\subsubsection{VAD Design Parameters}

The parameters that are used to design the 3D design of impeller is given below in tabular form. And this data is taken from [40]. 


\begin{tabular}{|l|l|l|}
\hline Diameter of impeller inlet & $\mathrm{D}_{\mathrm{i}}$ & $11 \mathrm{~mm}$ \\
\hline Diameter of impeller hub & $\mathrm{D}_{\mathrm{h}}$ & $4 \mathrm{~mm}$ \\
\hline Diameter of impeller outlet & $\mathrm{D}_{2}$ & $26 \mathrm{~mm}$ \\
\hline Width of impeller outlet & $\mathrm{B}_{2}$ & $3.5 \mathrm{~mm}$ \\
\hline Number of impeller blades & $\mathrm{Z}$ & 5,6 \\
\hline Wrap angle of impeller blades & $\varnothing$ & $100^{\circ}, 110^{\circ}, 120^{\circ}$ \\
\hline Diameter of volute circle & $\mathrm{D}_{3}$ & $30 \mathrm{~mm}$ \\
\hline Width of volute inlet & $\mathrm{B}_{3}$ & $5 \mathrm{~mm}$ \\
\hline Diameter of volute outlet & $\mathrm{D}^{\circ}$ & $10 \mathrm{~mm}$ \\
\hline
\end{tabular}

Table 4.1: List of design parameters of the VAD pump

\subsubsection{VAD 3D Design Illustrations}

So, by using the following parameters from the Table 4.1 3D structure of impeller has been build using SOLIDWORKS 2018 software.

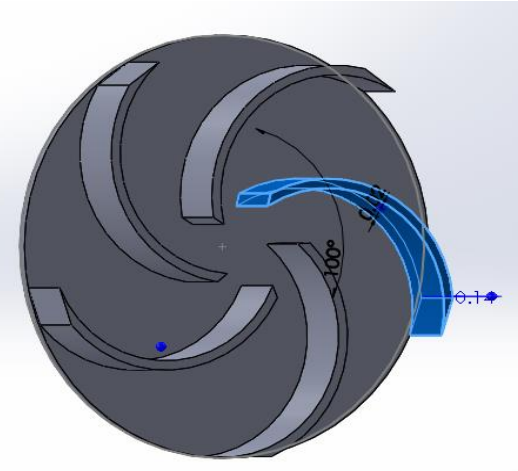

(a)

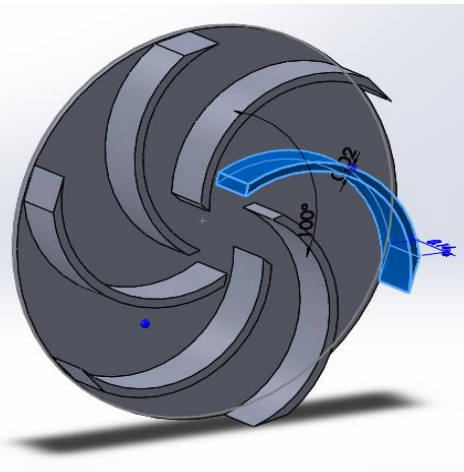

(b)

Figurer 4.1: (a) Impeller with $100^{\circ}$ wrap angle with 5 blades. (b) Impeller with $100^{\circ}$ wrap angle with 6 blades. 


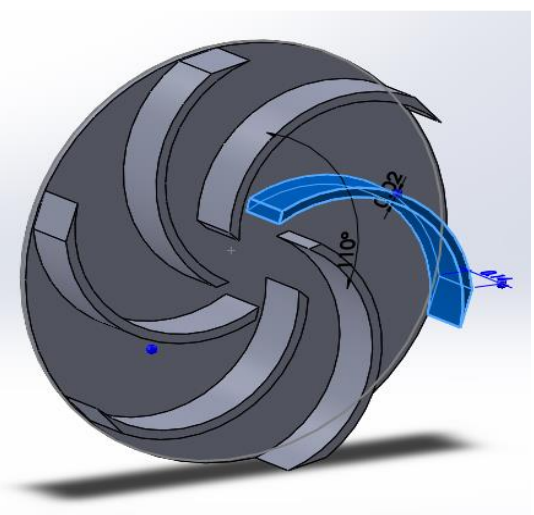

(a)

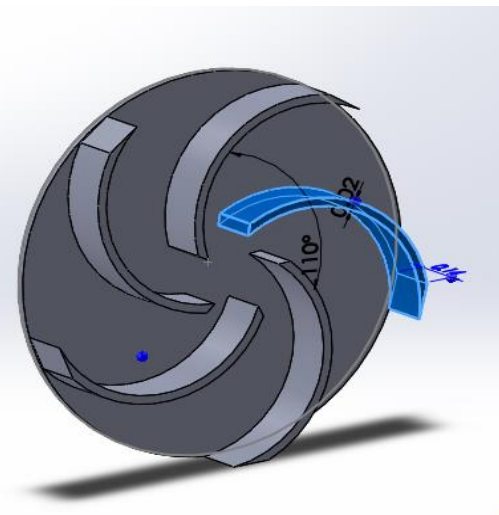

(b)

Figure 4.2: (a) Impeller with $110^{\circ}$ wrap angle with 6 blades. (b) Impeller with $110^{\circ}$ wrap angle with 5 blades.

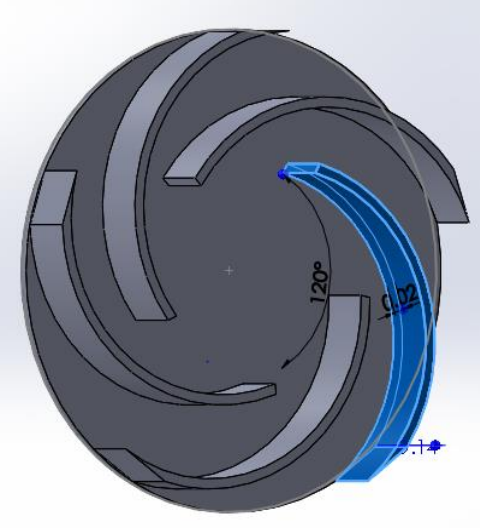

(a)

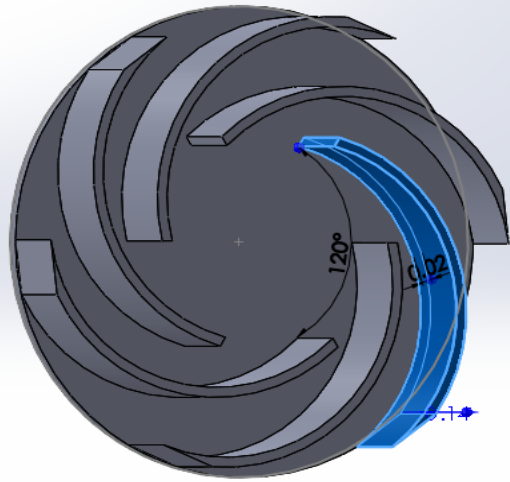

(b)

Figure 4.3: (a) Impeller with $120^{\circ}$ wrap angle with 5 blades. (b) Impeller with $120^{\circ}$ wrap angle with 6 blades. 


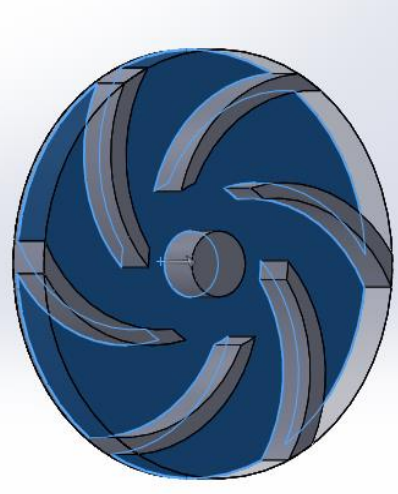

(a)

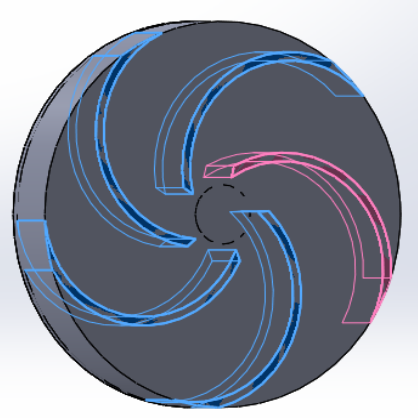

(b)

Figure 4.4: (a) Impeller with casing and eye of 6 blades. (b) Impeller with casing and eye of 5 blades.

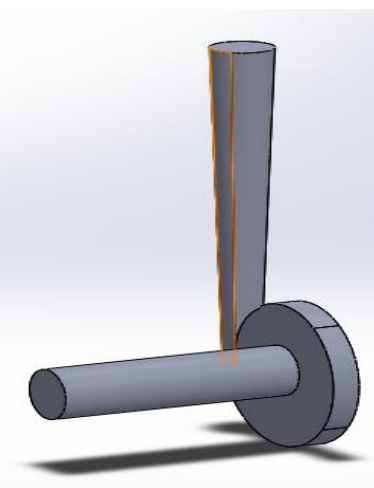

(a)

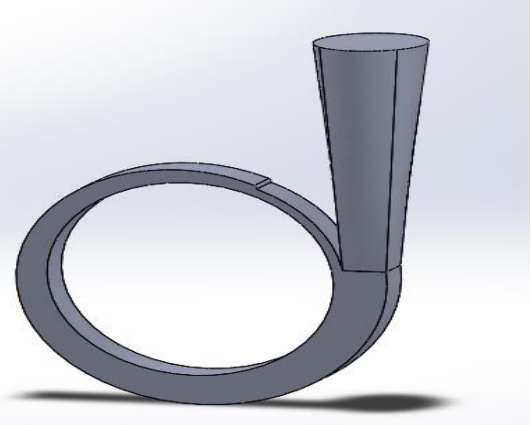

(b)

Figure 4.5: (a) Volute casing with inlet and outlet. (b) Volute casing without inlet. 


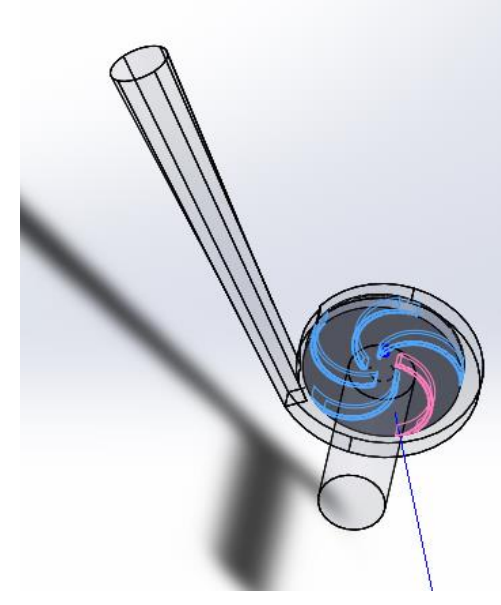

(a)

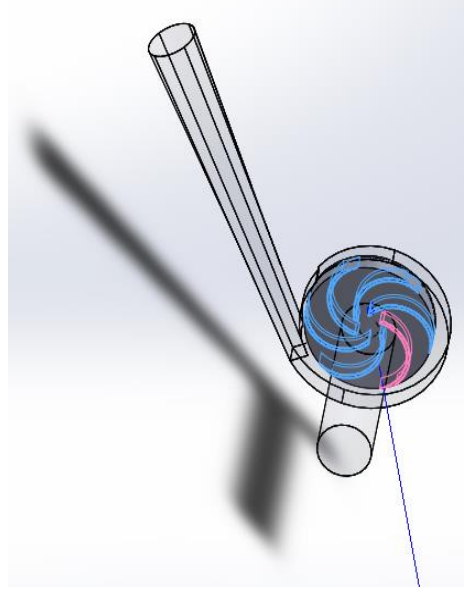

(b)

Figure 4.6: (a) Impeller of 5 blades with volute casing. (b) Impeller of 6 blades with volute casing.

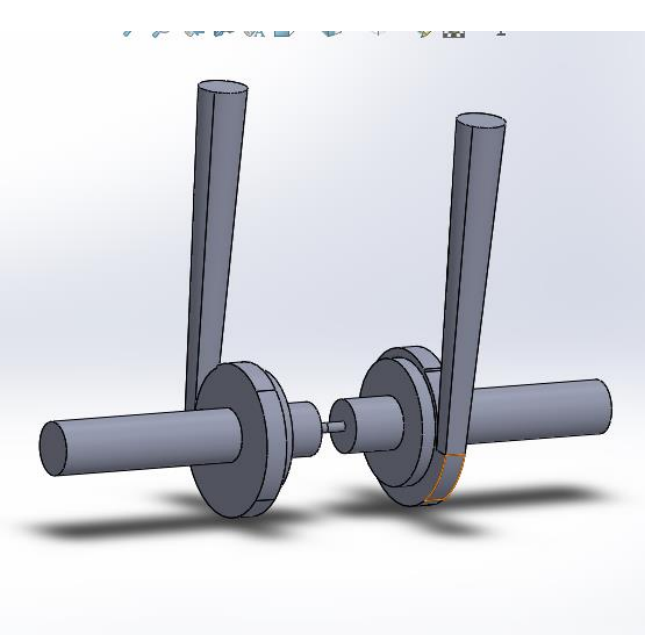

(a)

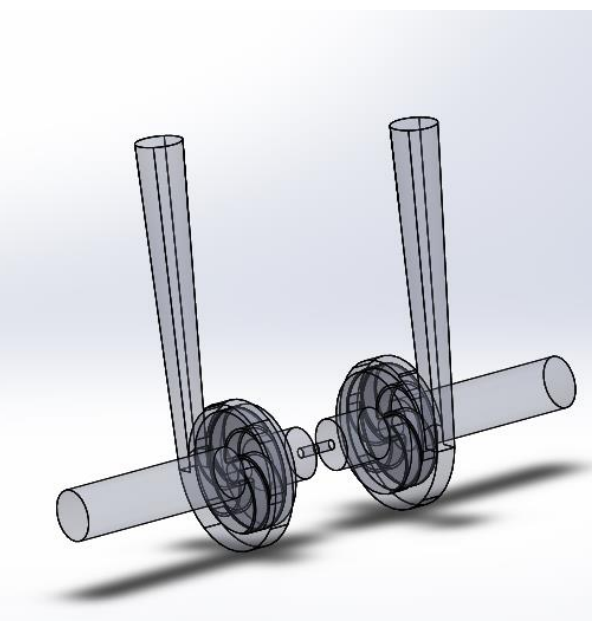

(b)

Figure 4.7: (a) Volute casing of inlet and outlet of Bi-VAD model (b) Impeller of the best design $\left(100^{\circ}\right.$ wrap angle with 6 blades) with volute casing. 


\subsection{Initial Single Sided VAD Simulation}

Initially, the simulation for the single sided VAD has been carried out using the ANSYS Fluent version 18.1 software to ensure whether the constructed impeller designs are capable of delivering the desired Head pressure and calculations were done to determine their corresponding values of Efficiency.

Once the initial simulations and calculations were done, the best design with Head pressure in the range $100 \mathrm{mmHg}-150 \mathrm{mmHg}$ [1] and highest Efficiency was selected and a more comprehensive simulation on the complete Bi-VAD model was conducted. Henceforth, the optimum rotating speed at which it can operate was constituted and an intricate analytical approach is made to conclude the overall pump performance.

\subsubsection{Geometry Establishment}

The final assembled fluid models of each impeller design with the volute casing from 4.2.3 are imported to the simulation domain of the ANSYS Fluent version 18.1 software for CFD analysis. The axis around which the impeller will rotate and the relative position of the volute is determined along with the naming of each part as named in the design section at the beginning of this Chapter. Boolean subtract function is further used to calculate the value of the Pressure difference between the inlet and outlet of the impeller that will be discussed later.

\subsubsection{Meshing}

Meshing is a vital part of the ANSYS Fluent 18.1 software that subdivides the arithmetic domain into small elements to improve calculation methods and obtain finite results throughout the entire simulation process being carried out. The mesh not only influences the accuracy but also the convergence as well as the speed of the solution.

The physical preference and solver preference are regulated in CFD and Ansys Fluent version 18.1 respectively as required for the simulations. The mesh method that is used for the 
constructed fluid designs is the ANSYS Fluent 18.1 adaptive mesh method [41] which ensures closely spaced mesh lines in regions demanding high resolution by mathematical solution of certain differential equations.

The mesh type that is being chosen for simulation purpose was the unstructured tetrahedralshaped elements [42]. Unstructured mesh generation modifies the node valence necessity, in a way that whatever the number of elements, they will eventually meet at a single node. This implements distinctive iterative smoothing algorithms generating proper mesh model with small angles avoiding skewed element angles for impeller blade tips and ensuring more accurate simulation results.

Setting of relevance options allows the control of the fineness of the mesh for the entire model. The greater the relevance, finer is the mesh ensuring results with increased accuracy. Hence, the value is set to 100 which is its maximum possible value.

The mesh generation is executed for the stator volute assembled with Impeller 1 of the 6 impeller designs and subsequently for Impeller2 to 6 at different values of mesh sizes. Mesh size is the mensuration of element size often used to regulate the element size distribution of a material while meshing and this being the reason causes an increase in the number of elements. Body Sizing is the method used to implement the different mesh sizes [41] in the simulations since it is useful while controlling the mesh size of multiple bodies and different quality meshes are required in certain bodies individually.

The resulting Pressure difference and Torque obtained during simulation along with the calculated value of the Head and Efficiency for Impeller1, 2, 3, 4, 5 and 6 are shown in Table $4.2,4.3,4.4,4.5,4.6$ and 4.7 respectively. The mesh structures of the volute, zoomed in volute to observe each element, impeller mesh design for 5 blades and impeller mesh design for 6 blades are shown in Figure 4.7, 4.8, 4.9 and 4.10 respectively. 


\begin{tabular}{|l|l|l|l|l|}
\hline Mesh Size (Pa) & $\begin{array}{l}\text { Pressure } \\
\text { difference }(\mathbf{P a})\end{array}$ & Torque (Nm) & Head (m) & Efficiency (\%) \\
\hline 0.15 & 22159.71 & 0.0082 & 2.14 & 53.33 \\
\hline 0.25 & 17179.86 & 0.0106765 & 1.66 & 31.99 \\
\hline 0.3 & 17329.095 & 0.00542408 & 1.67 & 63.44 \\
\hline 0.6 & 15863.836 & 0.00626685 & 1.53 & 50.38 \\
\hline
\end{tabular}

Table 4.2: The mesh sizes varied for Impeller1 ( $100^{\circ}$ wrap angle and 6 blades $)$ and the resulting pressure difference and torque obtained from simulation along with the calculated Head and Efficiency.

\begin{tabular}{|l|l|l|l|l|}
\hline $\begin{array}{l}\text { Mesh Size } \\
(\mathbf{m m})\end{array}$ & $\begin{array}{l}\text { Pressure } \\
\text { difference }(\mathbf{P a})\end{array}$ & Torque $(\mathbf{N m})$ & Head (m) & Efficiency (\%) \\
\hline 0.15 & 18024.73 & 0.00512037 & 1.74 & 70.09 \\
\hline 0.25 & 18045.99 & 0.002097 & 1.74 & 170.84 \\
\hline 0.3 & 17482.5 & 0.00563458 & 1.69 & 61.89 \\
\hline 0.6 & 16434.84 & 0.0275201 & 1.59 & 11.89 \\
\hline
\end{tabular}

Table 4.3: The mesh sizes varied for Impeller2 $\left(100^{\circ}\right.$ wrap angle and 5 blades $)$ and the resulting pressure difference and torque along with the calculated Head and Efficiency. 


\begin{tabular}{|l|l|l|l|l|}
\hline $\begin{array}{l}\text { Mesh Size } \\
(\mathbf{m m})\end{array}$ & $\begin{array}{l}\text { Pressure } \\
\text { difference }(\mathbf{P a})\end{array}$ & Torque $(\mathbf{N m})$ & Head $(\mathbf{m})$ & Efficiency (\%) \\
\hline 0.13 & 62137.75 & 5.93897 & 6.00 & 0.21 \\
\hline 0.15 & 18172.57 & $(-) 0.0167371$ & 1.76 & 21.54 \\
\hline 0.2 & 21433.13 & 0.01635 & 2.07 & 25.99 \\
\hline 0.3 & 19561.539 & 0.00675527 & 1.89 & 57.60 \\
\hline 0.6 & 15863.836 & 0.00626685 & 2.23 & 30.77 \\
\hline
\end{tabular}

Table 4.4: The mesh sizes varied for Impeller3 $\left(110^{\circ}\right.$ wrap angle and 6 blades $)$ and the resultant pressure and torque along with the calculated Head and Efficiency.

\begin{tabular}{|l|l|l|l|l|}
\hline $\begin{array}{l}\text { Mesh Size } \\
(\mathbf{m m})\end{array}$ & $\begin{array}{l}\text { Pressure } \\
\text { difference }(\mathbf{P a})\end{array}$ & Torque (Nm) & Head (m) & Efficiency (\%) \\
\hline 0.15 & 16764.03 & 0.00781753 & 1.62 & 42.81 \\
\hline 0.25 & 16762.75 & 0.00463115 & 1.62 & 72.16 \\
\hline 0.3 & 16948.29 & 0.00555022 & 1.64 & 60.78 \\
\hline
\end{tabular}

Table 4.5: The element sizes varied for Impeller4 ( $110^{\circ}$ wrap angle and 5 blades) and the resultant pressure difference and torque along with the calculated Head and Efficiency.

\begin{tabular}{|l|l|l|l|l|}
\hline $\begin{array}{l}\text { Mesh Size } \\
(\mathbf{m m})\end{array}$ & Pressure difference (Pa) & Torque (Nm) & Head (m) & Efficiency (\%) \\
\hline 0.15 & 22700.124 & 0.00849125 & 2.19 & 53.24 \\
\hline 0.20 & 23106.6 & 0.0061029 & 2.23 & 75.00 \\
\hline 0.25 & 22125.232 & 0.007375 & 2.14 & 59.54 \\
\hline 0.3 & 22821.422 & 0.00625033 & 2.21 & 72.90 \\
\hline 0.6 & 23188.349 & 0.00491326 & 2.24 & 93.69 \\
\hline
\end{tabular}

Table 4.6: The element sizes varied for Impeller5 $\left(120^{\circ}\right.$ wrap angle and 6 blades $)$ and the resultant pressure difference and torque along with the calculated Head and Efficiency 


\begin{tabular}{|l|l|l|l|l|}
\hline $\begin{array}{l}\text { Mesh Size } \\
(\mathbf{m m})\end{array}$ & $\begin{array}{l}\text { Pressure } \\
\text { difference } \\
(\mathbf{P a})\end{array}$ & Torque $(\mathbf{N m})$ & Head (m) & Efficiency (\%) \\
\hline 0.25 & 9355.737 & 0.0893206 & 0.90 & 2.07 \\
\hline 0.3 & 19386.52 & 0.155023 & 1.87 & 2.48 \\
\hline 0.6 & 18235.75 & 0.0079792 & 1.76 & 45.50 \\
\hline
\end{tabular}

Table 4.7: The mesh sizes varied for Impeller6 ( $120^{\circ}$ wrap angle and 5 blades $)$ and the resultant pressure difference and torque along with the calculated Head and Efficiency.

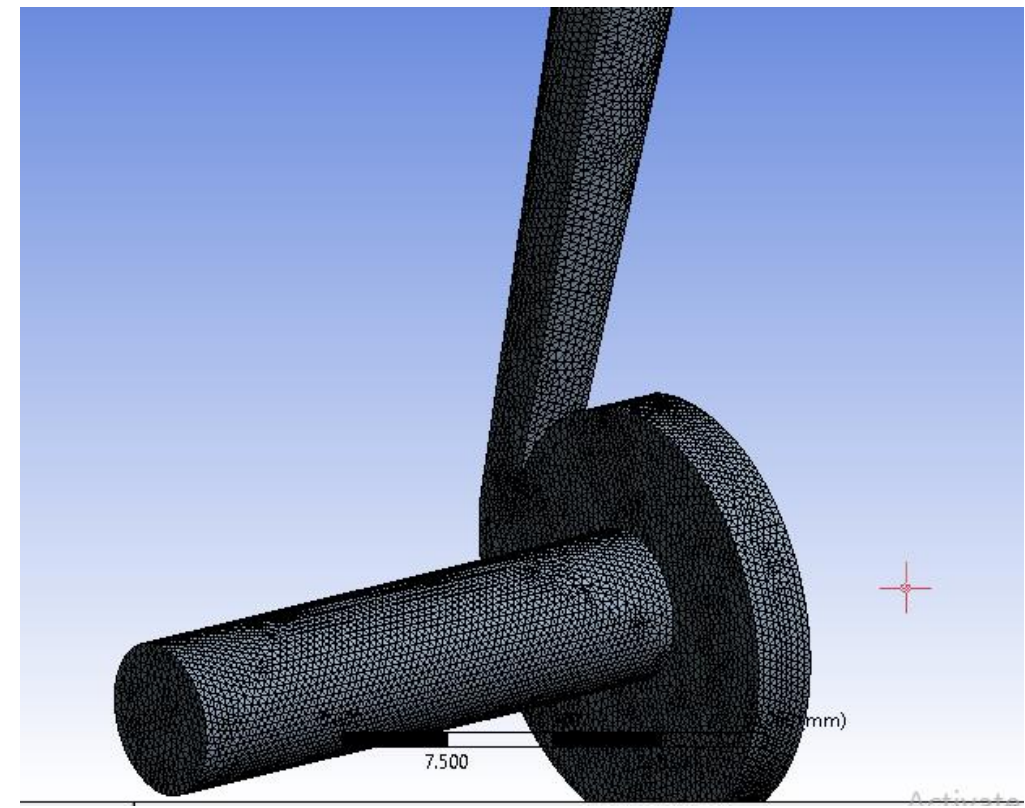

Figurer 4.8: Volute casing mesh used for the 6 impeller designs. 


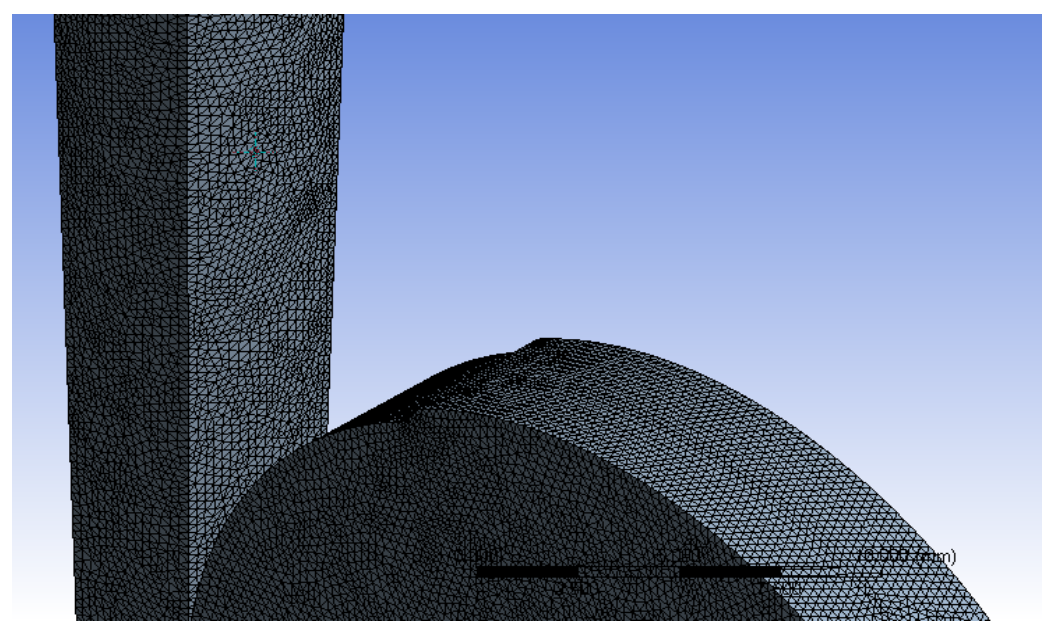

Figurer 4.9: Zoomed in volute casing to observe mesh.

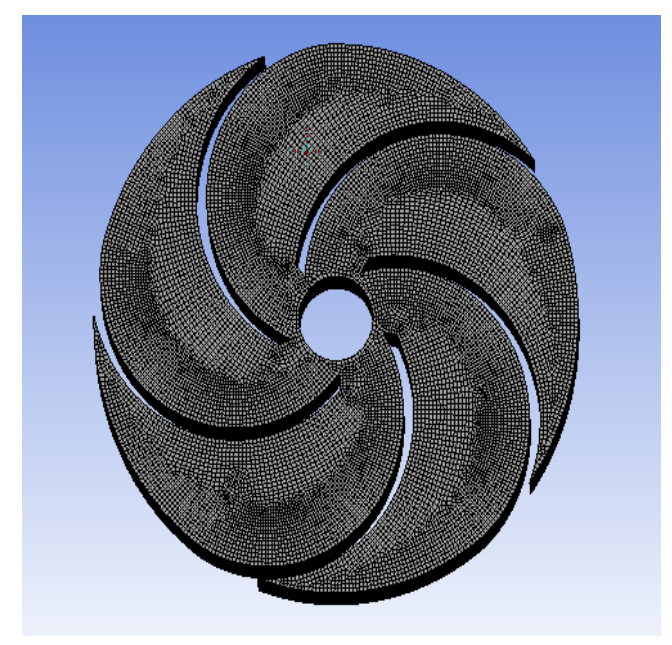

(a)

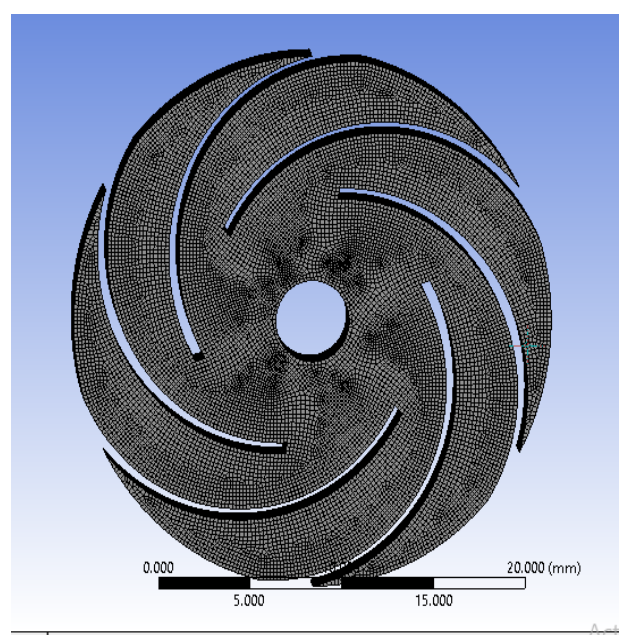

(b)

Figurer 4.10: (a) Impeller mesh design for 5 blades. (b) Impeller mesh design for 6 blades.

From the above tables, it is prominent that for mesh size of $0.3 \mathrm{~mm}$, the calculated Efficiencies for all the impellers comes up to be the highest and hence, this mesh size is considered as the standard for the further simulations shown later in this Chapter where the Flow rates of the blood is varied to again find out that for which one comes the optimum Efficiency. 


\subsubsection{Input Properties}

The operating environment of the pump being blood, the fluid type selected reflected blood since the blood density (1056 kg/m3) [1] and blood viscosity (0.004 Pa.s) [1] was applied as input in its operating conditions. Blood is a non-Newtonian fluid and its viscosity varies depending on how much shear stress is placed on it [6]. The "shear-thinning" property causes the shear rate to increase above 100 per second making it behave like a Newtonian fluid, exactly for which it is modelled as a Newtonian fluid during the simulations provided blood pumps and aorta have a high shear stress rate nature.

\subsubsection{Boundary Conditions}

The boundary conditions being applied in order to conduct the initial simulation are defined objectively to simulate and calculate the value of Efficiency for each impeller design by varying the values of the Flow rate. The mass flow inlet set to normal at the boundary can be calculated using the formula [44]:

$$
\text { Mass Flow rate }=Q \times \text { Density }=0.000083 \frac{\mathrm{m}^{3}}{\mathrm{~s}} \times 1056 \frac{\mathrm{kg}}{\mathrm{m}^{3}}=0.088 \frac{\mathrm{kg}}{\mathrm{s}}
$$

Where, the parameter, Q is the Flow rate of the fluid flowing [44] and Density is the density of blood.

Since the value of the flow rate between the inlet and the outlet of the impellers are equivalent to each other, the Mass Flow rate value of $0.088 \mathrm{~kg} / \mathrm{s}$ was set to normal at the boundary of the inlet only. The stationary wall part is named the volute casing whereas the rotational part is named the impeller and fluid casing previously in the geometry section. Figure 4.12 shows the interface at the connecting surface between rotating and stationary domain.

The simulation for varying Mass Flow rates were carried out for 50 iterations for previously stated six models of impellers and resulting value of Head and Efficiency are recorded in Table 4.8, Table 4.9, Table 4.10, Table 4.11, Table 4.12 and Table 4.13 for each of the impellers, 
Impeller1, 2, 3, 4, 5 and 6 respectively and the interface at the connecting surface between rotating and stationary domain is shown in Figure 4.11

\begin{tabular}{|l|l|l|l|l|}
\hline $\begin{array}{l}\text { Mass Flow rate } \\
(\mathbf{k g} / \mathbf{s})\end{array}$ & $\begin{array}{l}\text { Pressure } \\
\text { Difference } \\
\mathbf{( P a )}\end{array}$ & Torque $(\mathbf{N m})$ & Head (m) & Efficiency (\%) \\
\hline 0.029 & 5081.598 & 0.00083534 & 0.491 & 3.13 \\
\hline 0.044 & 8676.593 & 0.00251571 & 0.838 & 60.57 \\
\hline 0.059 & 12115.49 & 0.00499707 & 1.17 & 45.77 \\
\hline 0.073 & 17329.095 & 0.00542408 & 1.67 & 40.19 \\
\hline 0.088 & 22484.4 & 0.00618922 & 2.17 & 63.44 \\
\hline 0.103 & 26760.1 & 0.00989024 & 2.59 & 84.17 \\
\hline 0.117 & 34475.55 & 0.0076888 & 3.33 & 71.98 \\
\hline 0.132 & & & 133.85 \\
\hline
\end{tabular}

Table 4.8: Calculated Head and Efficiency at different Flow rates for corresponding Pressure Difference and Torque of Impeller1 with $100^{\circ}$ wrap angle and 6 blades. 


\begin{tabular}{|l|l|l|l|l|}
\hline $\begin{array}{l}\text { Mass Flow rate } \\
(\mathbf{k g} / \mathbf{s})\end{array}$ & $\begin{array}{l}\text { Pressure } \\
\text { Difference } \\
\mathbf{( P a )}\end{array}$ & Torque $(\mathbf{N m})$ & Head $(\mathbf{m})$ & Efficiency $(\%)$ \\
\hline 0.029 & 2433.086 & 0.0015243 & 0.235 & 10.66 \\
\hline 0.044 & 5032.219 & 0.0024012 & 0.486 & 20.79 \\
\hline 0.059 & 7357.326 & 0.00516464 & 0.711 & 18.92 \\
\hline 0.073 & 12203.77 & 0.0047606 & 1.18 & 42.56 \\
\hline 0.088 & 17482.5 & 0.00563458 & 1.69 & 61.89 \\
\hline 0.103 & 22290.07 & 0.00703558 & 2.15 & 73.22 \\
\hline 0.117 & 30946.08 & 0.00275349 & 2.99 & 298.26 \\
\hline 0.132 & 36292.26 & 0.0059299 & 3.51 & 183.06 \\
\hline
\end{tabular}

Table 4.9: Calculated Head and Efficiency at different Flow rates for corresponding Pressure Difference and Torque of Impeller2 with $100^{\circ}$ wrap angle and 5 blades.

\begin{tabular}{|l|l|l|l|l|}
\hline $\begin{array}{l}\text { Mass Flow rate } \\
(\mathbf{k g} / \mathbf{s})\end{array}$ & $\begin{array}{l}\text { Pressure } \\
\text { Difference } \\
(\mathbf{P a})\end{array}$ & Torque $(\mathbf{N m})$ & Head (m) & Efficiency $(\%)$ \\
\hline 0.029 & $(-) 786.7602$ & $6.2 \times 10^{009}$ & 0.076 & $8.46 \times 10^{-13}$ \\
\hline 0.044 & 4456.425 & 0.0019194 & 0.431 & 23.15 \\
\hline 0.059 & 9236.418 & 0.00491546 & 0.893 & 24.95 \\
\hline 0.073 & 11811.48 & 0.00690045 & 1.14 & 28.34 \\
\hline 0.088 & 19561.539 & 0.00675527 & 1.89 & 57.60 \\
\hline 0.103 & 19766.208 & 0.00993724 & 1.91 & 46.15 \\
\hline 0.117 & 20076.672 & 0.0324846 & 1.94 & 16.40 \\
\hline 0.132 & 21111.552 & 0.00764043 & 2.04 & 82.50 \\
\hline
\end{tabular}

Table 4.10: Calculated Head and Efficiency at different Flow rates for corresponding Pressure Difference and Torque of Impeller3 with $110^{\circ}$ wrap angle and 6 blades. 


\begin{tabular}{|l|l|l|l|l|}
\hline Mass Flow rate & $\begin{array}{l}\text { Pressure } \\
\text { Difference } \\
(\mathbf{P a})\end{array}$ & Torque $(\mathbf{N m})$ & Head $(\mathbf{m})$ & Efficiency $(\%)$ \\
\hline 0.029 & 2666.93 & 0.00131459 & 0.258 & 13.45 \\
\hline 0.044 & 3941.484 & 0.00433584 & 0.381 & 9.01 \\
\hline 0.059 & 5416.104 & 0.00742004 & 0.523 & 9.68 \\
\hline 0.073 & 15185.24 & 0.000652763 & 1.47 & 388.28 \\
\hline 0.088 & 16948.29 & 0.00555022 & 1.64 & 60.78 \\
\hline 0.103 & 23461.94 & 0.00388405 & 2.27 & 139.88 \\
\hline 0.117 & 26668.89 & 0.0147938 & 2.58 & 47.82 \\
\hline 0.132 & 35388.54 & 0.00486763 & 3.42 & 216.67 \\
\hline
\end{tabular}

Table 4.11: Calculated Head and Efficiency at different Flow rates for corresponding Pressure Difference and Torque of Impeller4 with $110^{\circ}$ wrap angle and 5 blades.

\begin{tabular}{|l|l|l|l|l|}
\hline $\begin{array}{l}\text { Mass Flow rate } \\
(\mathbf{k g} / \mathbf{s})\end{array}$ & $\begin{array}{l}\text { Pressure } \\
\text { Difference } \\
\mathbf{( P a )}\end{array}$ & Torque $(\mathbf{N m})$ & Head $(\mathbf{m})$ & Efficiency $(\%)$ \\
\hline 0.029 & 3206.552 & 0.00168166 & 0.310 & 12.64 \\
\hline 0.044 & 6574.796 & 0.00318274 & 0.635 & 20.60 \\
\hline 0.059 & 11153.95 & 0.00353747 & 1.08 & 41.96 \\
\hline 0.073 & 16344.77 & 0.00475032 & 1.58 & 56.78 \\
\hline 0.088 & 22821.422 & 0.00625033 & 2.21 & 72.90 \\
\hline 0.103 & 29639.26 & 0.00793158 & 2.86 & 86.75 \\
\hline 0.117 & 38574.14 & 0.0073579 & 3.73 & 138.96 \\
\hline 0.132 & 47437.93 & 0.00937692 & 4.58 & 150.64 \\
\hline
\end{tabular}

Table 4.12: Calculated Head and Efficiency at different Flow rates for corresponding Pressure Difference and Torque of Impeller 5 with $120^{\circ}$ wrap angle and 6 blades. 


\begin{tabular}{|l|l|l|l|l|}
\hline $\begin{array}{l}\text { Mass Flow rate } \\
(\mathbf{k g} / \mathbf{s})\end{array}$ & $\begin{array}{l}\text { Pressure } \\
\text { Difference } \\
\mathbf{( P a )}\end{array}$ & Torque $(\mathbf{N m})$ & Head (m) & Efficiency (\%) \\
\hline 0.029 & 5930.839 & $(-) 0.048571$ & 0.573 & 0.811 \\
\hline 0.044 & 6126.4896 & 0.188556 & 0.592 & 0.323 \\
\hline 0.059 & 13039.488 & 0.0177353 & 1.26 & 9.76 \\
\hline 0.073 & 17385.984 & 0.78422 & 1.68 & 0.369 \\
\hline 0.088 & 19386.52 & 0.155023 & 1.87 & 2.48 \\
\hline 0.103 & $(-) 28291.78$ & 0.209094 & 2.73 & 3.14 \\
\hline 0.117 & 32288.256 & $(-) 0.393757$ & 3.12 & 2.17 \\
\hline 0.132 & 33633.6 & 1.45765 & 3.25 & 0.688 \\
\hline
\end{tabular}

Table 4.13: Calculated Head and Efficiency at different Flow rates for corresponding Pressure Difference and Torque of Impeller6 with $120^{\circ}$ wrap angle and 5 blades.

From the above tables, it can be predicted under operating condition of Mass Flow rate of $0.088 \mathrm{~kg} / \mathrm{s}$, all the six designed impellers shows the perfect, finest combination of the Efficiency as well as the required value of Head.

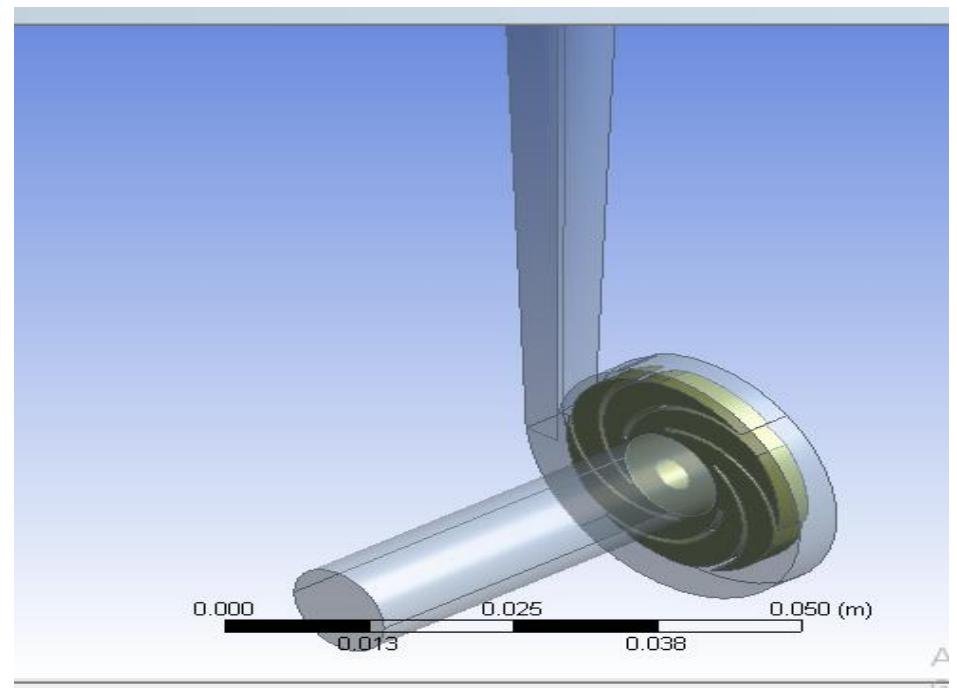

Figure 4.11: Interface at the connecting surface between rotating and stationary domain. 


\subsubsection{Setup and Discussions}

Setup of general parameters is done by inputting pressure-based setup with steady time scale at absolute velocity formulation which allows the simulation to generate a pressure-based scale diagram at a particular time from where the pressure difference between the inlet and outlet of the fluid model is calculated.

The model selected remains to be the $k-\varepsilon$ model (i.e., turbulence model) as discussed previously in Chapter 3 .

While choosing the materials, the default water parameters from the Fluent Database of the ANSYS Fluent version 18.1 software are manipulated to match the consistency of blood (i.e., density (1056 kg/m3) [1] and viscosity (0.004 Pa.s) [1]).

Since there are multiple volumes with varying motions in the complicated models constructed, cell zone conditions are set individually for each part. The volute casing set at no motion since it must stay stationary and then the impeller and fluid casing set at a frame motion with angular velocity of $4000 \mathrm{rpm}$ [1] as it is the rotating part.

In the boundary conditions setup, type of inlet chosen is the Mass Flow inlet and Mass Flow rate of $0.088 \mathrm{~kg} / \mathrm{s}$ was set to normal at the boundary of the inlet with the aid of direction specification method. For the wall part that is the volute casing, wall motion is set to moving wall whereas motion related to the adjacent cell zone consisting of the part of the impeller and fluid casing was set to rotational as required.

Lastly, using standard initializations, the calculations are run for 50 iterations which is enough because continuity goes to $1 / 100$. 


\subsubsection{Selection of Best Impeller for RVAD and Bi-VAD Simulation}

The simulation for the LVAD was done in two sections. First the simulation was done for different mesh sizes for each of the 6 designed impellers from where the best suitable mesh size where all the six impellers showed appropriate values of Head and Efficiency was decided (i.e., $0.3 \mathrm{~mm}$ ) to carry out further simulations. In section 2 , the simulation was done for 7 different Flow rates for each impeller under the mesh size of $0.3 \mathrm{~mm}$. Hence, approximately total 72 numbers of simulations were done for all the six impellers. From the simulation values the Pressure difference of the fluid flowing in between the inlet and outlet of the impeller as well as the resulting generated value of Torque of the impeller were established. Utilizing these data, the values of the Head and Efficiency of each of the impeller was calculated (detailed calculation is shown in Chapter 5) for the ideal best mesh size and varying Flow rates for each of the impellers. From all the calculated data of the impellers with mesh size of $0.3 \mathrm{~mm}$, varying Flow rates and the Pressure difference and Torque obtained from the simulation, the data calculated for each of the impeller was compared to the other remaining impellers and the specific flow rate where all the impellers showed an appropriate and suitable value of Head and Efficiency was selected as the best value of Flow rate (i.e., $0.3 \mathrm{~m}^{3} / \mathrm{h}$ ). Therefore, the data of the Head and Efficiency was analyzed and compared for all the 6 designed impellers of LVAD with a mesh size of $0.3 \mathrm{~mm}$ and $0.3 \mathrm{~m}^{3} / \mathrm{h}$ and the impeller design that showed the finest combination of the value of Head and Efficiency at the chosen best value of mesh size and Flow rate amongst others was selected and handed - down for the RVAD and Bi-VAD simulation.

In order to select one optimum design both the Head and Efficiency was taken into consideration. The first priority was the Efficiency, with higher value of Efficiency of the impeller, the performance rate of the pump increases. But the Head pressure corresponding to the selected Efficiency should fall in the range of the Head pressure of an actual heart environment. Different authors have chosen different Head for LVAD centrifugal pump as reference point. For this thesis 100-150 mmHg was chosen as reference [1]. Again, the higher Head value within the range is considered to be better. 


\begin{tabular}{|l|l|l|l|l|l|l|l|l|}
\hline $\begin{array}{l}\text { Design } \\
\text { name }\end{array}$ & $\begin{array}{l}\text { Mesh } \\
\text { size } \\
(\mathrm{mm})\end{array}$ & $\begin{array}{l}\text { Element } \\
\text { no. }\end{array}$ & Node no & $\begin{array}{l}\text { Pressure } \\
\text { difference } \\
(\mathrm{Pa})\end{array}$ & Torque $(\mathrm{Nm})$ & $\begin{array}{l}\text { Head } \\
(\mathrm{m})\end{array}$ & $\begin{array}{l}\text { Head } \\
(\mathrm{mmHg})\end{array}$ & $\begin{array}{l}\text { Efficiency } \\
(\%)\end{array}$ \\
\hline Impeller1 & 0.30 & 266070 & 689196 & 17329.095 & 0.00542408 & 1.67 & 122.81 & 63.44 \\
\hline Impeller 2 & 0.30 & 2614791 & 683596 & 17482.5 & 0.00563458 & 1.69 & 124.27 & 61.89 \\
\hline Impeller 3 & 0.30 & 2317530 & 799141 & 19561.539 & 0.00675527 & 1.89 & 138.95 & 57.60 \\
\hline Impeller 4 & 0.30 & 2642436 & 682317 & 16948.29 & 0.005505022 & 1.64 & 120.60 & 60.78 \\
\hline Impeller 5 & 0.30 & 2716706 & 690987 & 22821.422 & 0.00625033 & 2.21 & 162.52 & 72.90 \\
\hline Impeller 6 & 0.30 & 2622578 & 675461 & 19386.52 & 0.155023 & 1.87 & 137.51 & 2.48 \\
\hline
\end{tabular}

Table 4.14: Summary Table of Head and Efficiency of Impeller design 1 to 6 at optimum mesh size of $0.30 \mathrm{~mm}$ and flow rate of $0.30 \mathrm{~m}^{3} / \mathrm{h}$.

From Table 4.14 it clearly shows that Impeller5 has the highest value of Efficiency amongst the other impellers which is $72.90 \%$ but the corresponding value of Head is $2.21 \mathrm{~m}(162.52 \mathrm{mmHg})$. This Head value exceeds the selected range of Head pressure. So, this design was rejected for further simulation. Impeller 1 and 2 has close Efficiency values respectively $63.44 \%$ and $61.89 \%$. Even though Impeller2 has higher Head 1.69m (124.27 mmHg) than Impeller1, 1.67(122.81 $\mathrm{mmHg}$ ), Impeller1 was chosen to be the best design for having higher Efficiency than Impeller2.

Impeller1 was then used to perform the simulation for RVAD and eventually for Bi-VAD.

\subsection{RVAD Simulation}

The RVAD simulation is done using the best model predicted from the initial LVAD simulations similarly to that of LVAD using ANSYS Fluent version 18.1 software with the aid of the same principles. The model with the highest Efficiency which is Impeller1 with $100^{\circ}$ wrap angle and 6 blades providing Head in the desired range is considered to be the best design. 


\subsubsection{Geometry Establishment}

The fluid model of RVAD is generated following the same principles as the LVAD. The resulting geometry model is shown in Figure 4.12.

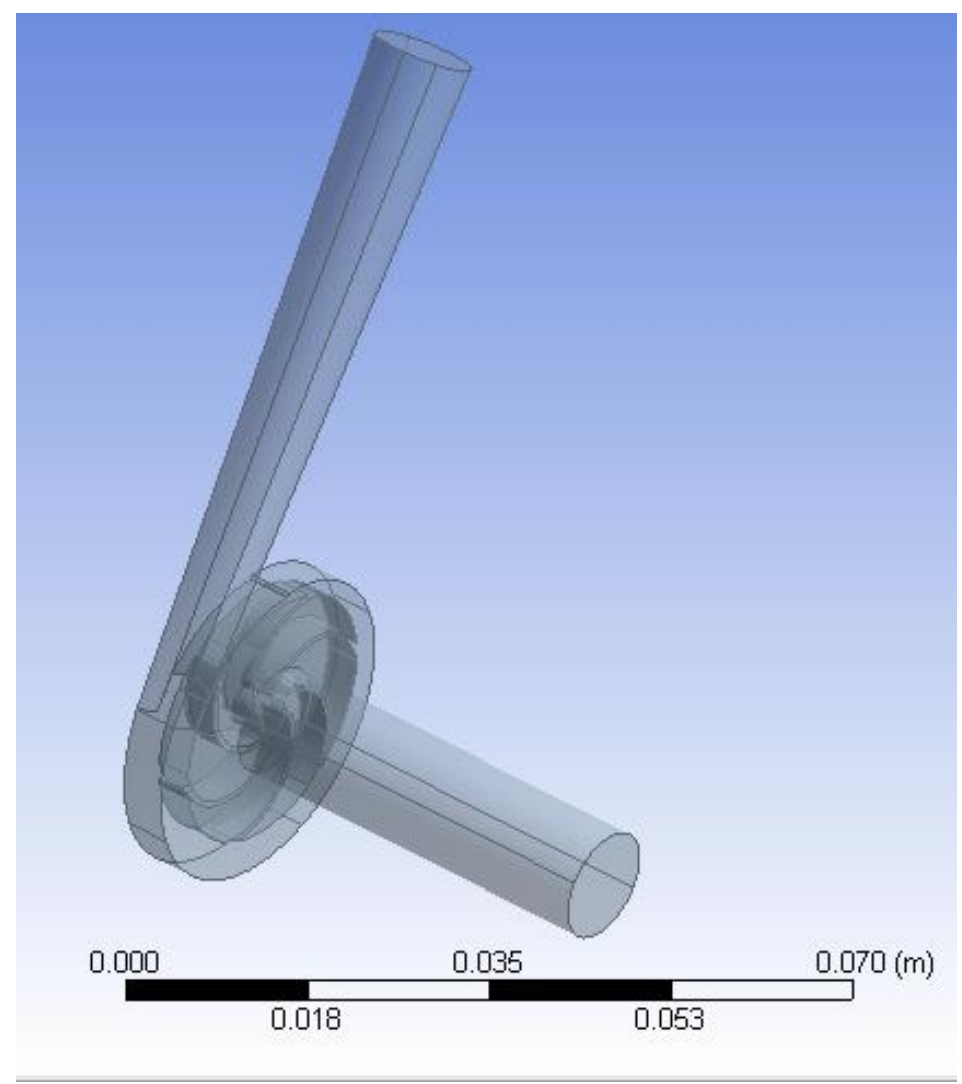

Figurer 4.12: RVAD geometry model of the best design of Impeller1 with $100^{\circ}$ wrap angle and 6 blades.

\subsubsection{Meshing}

The mesh model of RVAD is generated following the same principles as the LVAD. The mesh size used is $0.3 \mathrm{~mm}$ as it gives the best Efficiency according to the prior LVAD calculations. The resulting developed mesh model for the impeller is shown in Figure 4.13. 


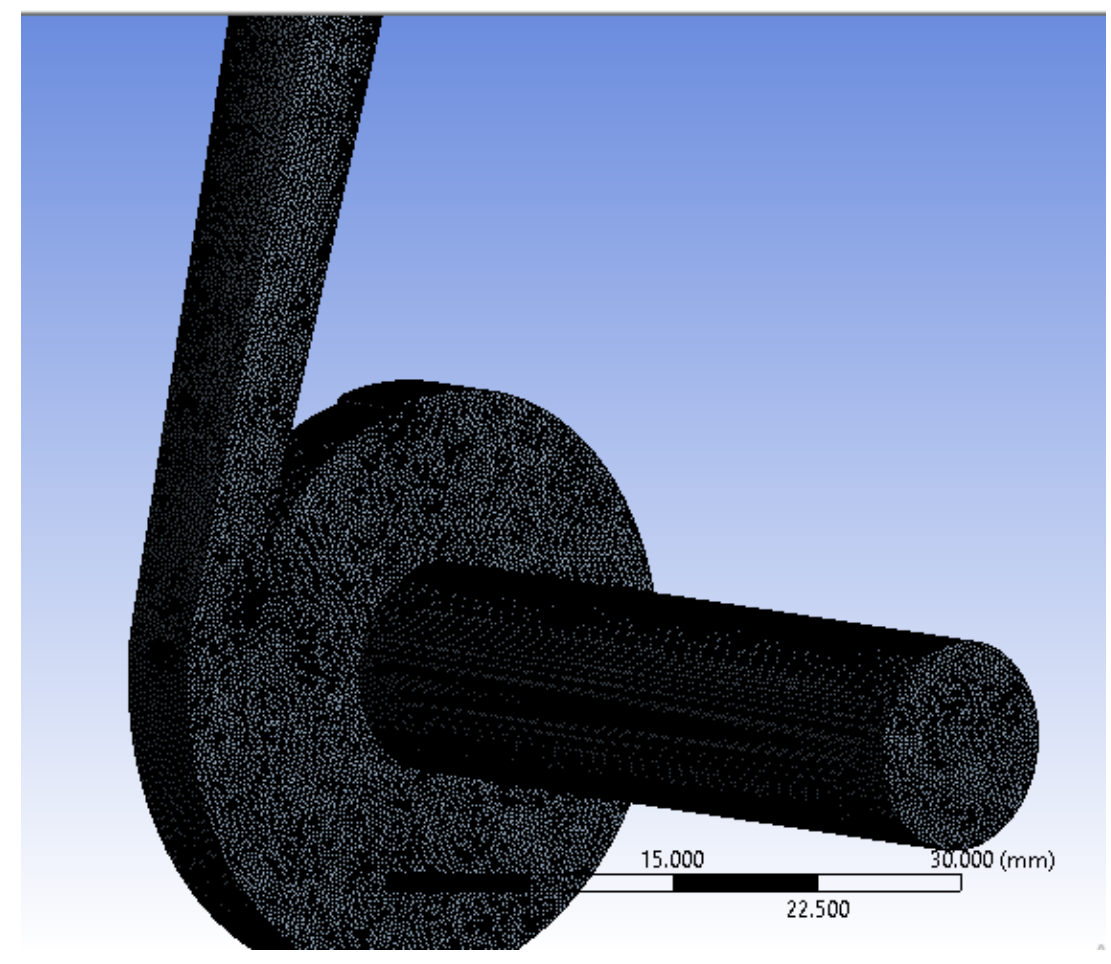

Figure 4.13: RVAD mesh model of the best design of impeller design 1 with $100^{\circ}$ wrap angle and 6 blades.

\subsubsection{Input Properties}

The input properties used in RVAD are same as LVAD. That is, blood density $(1056 \mathrm{~kg} / \mathrm{m} 3)$ [1] and blood viscosity (0.004 Pa.s) [1].

\subsubsection{Boundary Conditions}

The boundary conditions applied during the simulation of the RVAD are also same as the LVAD except for one. The inlet velocity for LVAD and RVAD will be different due to different requirements of the speed of blood for the right and left ventricle. Hence, the velocity is set at $3054 \mathrm{rpm}$ [1] for RVAD. 


\subsubsection{Setup and Results}

The setup of RVAD is once again same as that of LVAD except for the boundary conditions mentioned. In the boundary condition setup, the volute casings are set at no motion whereas the impeller and fluid casing set at a frame motion with angular velocity of $3054 \mathrm{rpm}$ [1] for RVAD.

The calculated Head and Efficiency at flow rate $0.008 \mathrm{~kg} / \mathrm{s}$ and mesh size $0.3 \mathrm{~mm}$ for corresponding Pressure Difference and Torque of Impeller1 with $100^{\circ}$ wrap angle and 6 blades are shown in Table 4.15.

\begin{tabular}{|l|l|l|l|l|l|l|}
\hline $\begin{array}{l}\text { Best Impeller } \\
\text { Design }\left(\mathbf{1 0 0 ^ { \circ }}\right.\end{array}$ & $\begin{array}{l}\text { Mass } \\
\text { Flow }\end{array}$ & $\begin{array}{l}\text { Mesh } \\
\text { size } \\
\text { wrap angle } \\
\text { and 6 blades })\end{array}$ & $\begin{array}{l}\text { Pressure } \\
(\mathbf{m m})\end{array}$ & $\begin{array}{l}\text { Torque }(\mathbf{N m}) \\
(\mathbf{P a})\end{array}$ & $\begin{array}{l}\text { Head } \\
(\mathbf{m})\end{array}$ & $\begin{array}{l}\text { Efficiency } \\
(\%)\end{array}$ \\
\hline RVAD & 0.088 & 0.3 & 16427.8 & 0.00503789 & 1.59 & 85.09 \\
\hline
\end{tabular}

Table 4.15: Calculated Head and Efficiency of RVAD for corresponding Pressure Difference and Torque of Impeller 1 with $100^{\circ}$ wrap angle and 6 blades.

\subsection{Bi-VAD Simulation}

The final Bi-VAD simulation is done using the best model predicted from the initial LVAD simulations similarly to that of LVAD using ANSYS Fluent version 18.1 software with the aid of the same principles. The model with the highest Efficiency which is Impellerl with $100^{\circ}$ wrap angle and 6 blades providing Head in the desired range is considered to be the best design. 


\subsubsection{Geometry Establishment}

The fluid model of Bi-VAD is generated following the same principles as the LVAD. The resulting developed geometry model for the impeller is shown in Figure 4.14.

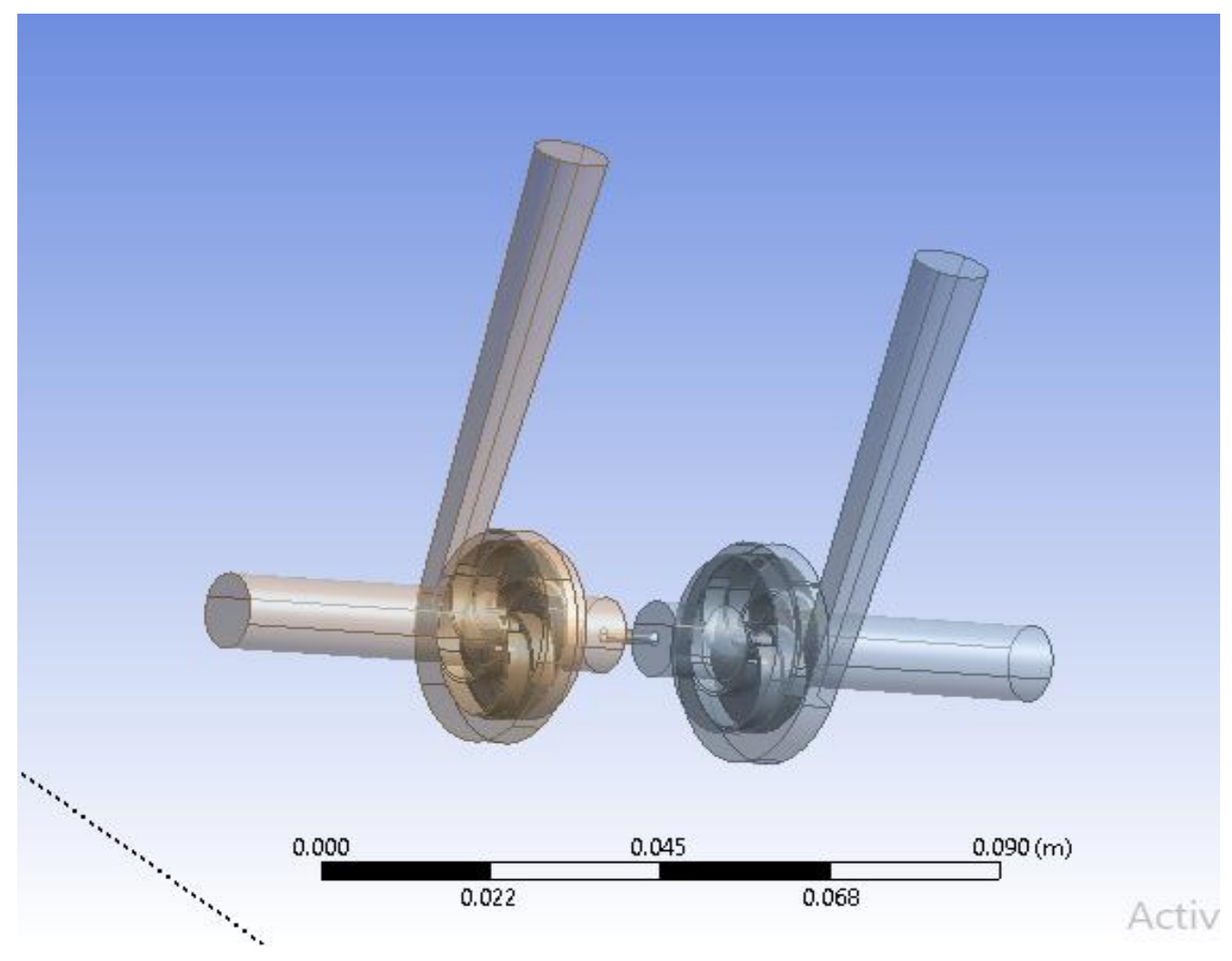

Figure 4.14: Final Bi-VAD geometry model of the best design.

\subsubsection{Meshing}

The mesh model of Bi-VAD is generated following the same principles as the LVAD. The mesh size used is $0.3 \mathrm{~mm}$ as it gives the best Efficiency according to prior LVAD calculations. The resulting developed mesh model for the impeller is shown in Figure 4.15. 


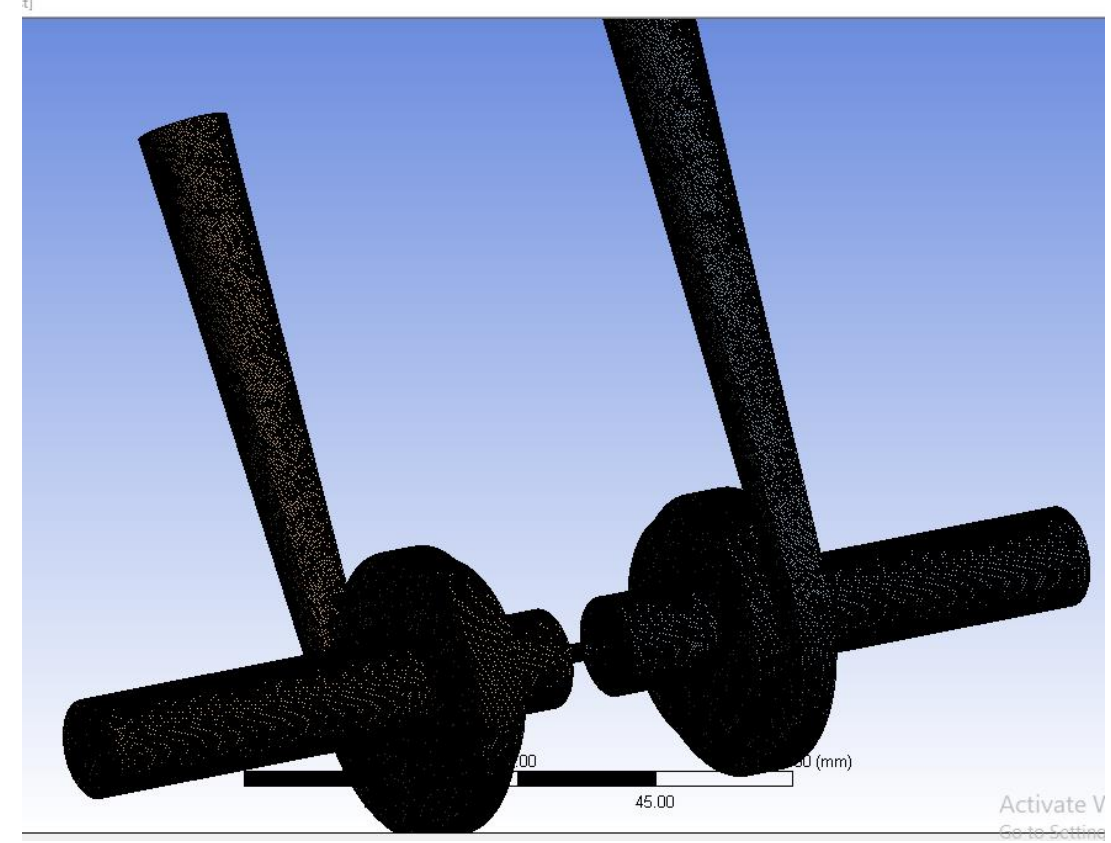

Figure 4.15: Final Bi-VAD mesh model of the best design

\subsubsection{Input Properties}

The input properties used in Bi-VAD are the same as LVAD. That is, blood density (1056 $\mathrm{kg} / \mathrm{m} 3)$ [1] and blood viscosity (0.004 Pa.s) [1].

\subsubsection{Boundary Conditions}

The boundary conditions applied during the simulation of the Bi-VAD are also similar to the boundary conditions applied during the simulation of the LVAD except for one. The inlet velocity for the LVAD and RVAD will be different due to different requirements of the speed of blood for the right and left ventricle. Hence, the velocities are set at $4000 \mathrm{rpm}$ [1] for LVAD and $3054 \mathrm{rpm}$ [1] for RVAD. 


\subsubsection{Setup and Results}

The setup of Bi-VAD is again similar to that of LVAD except for the boundary conditions mentioned. In the boundary condition setup, the volute casings are set at no motion whereas the impeller and fluid casing set at a frame motion with angular velocity of $4000 \mathrm{rpm}$ [1] for LVAD and $3054 \mathrm{rpm}$ [1] for RVAD.

The calculated Head and Efficiency at flow rate $0.008 \mathrm{~kg} / \mathrm{s}$ and mesh size $0.3 \mathrm{~mm}$ for corresponding Pressure Difference and Torque of Impeller 1 with $100^{\circ}$ wrap angle and 6 blades are shown in Table 4.16

\begin{tabular}{|l|l|l|l|l|l|}
\hline $\begin{array}{l}\text { Best } \\
\text { Impeller } \\
\text { Design }\left(\mathbf{1 0 0} 0^{\circ}\right.\end{array}$ & $\begin{array}{l}\text { Mass } \\
\text { Flow } \\
\text { rate } \\
\text { 6rap angle, }\end{array}$ & $\begin{array}{l}\text { Pressure } \\
\text { Difference } \\
(\mathbf{k g} / \mathbf{s})\end{array}$ & $\begin{array}{l}\text { Torque }(\mathbf{N m}) \\
(\mathbf{m})\end{array}$ & & \\
\hline $\begin{array}{l}\text { LVAD } \\
\text { RVAD }\end{array}$ & 0.088 & 10371.37 & 0.00355263 & 1.00 & 57.85 \\
\hline
\end{tabular}

Table 4.16: Calculated Head and Efficiency of Bi-VAD for corresponding Pressure Difference and Torque of Best Impeller design with $100^{\circ}$ wrap angle and 6 blades.

\subsection{Conclusion}

Six impeller designs along with a volute has been constructed with varying wrap angles of $100^{\circ}, 110^{\circ}$ and $120^{\circ}$ for blade numbers -5 and 6 for LVAD. Further simulations are carried out at different mesh sizes to determine that the optimum mesh size at which the Efficiency of the impeller is the highest while Head is also in the desired range is $0.3 \mathrm{~mm}$. Next, keeping this Head constant, further simulations carried out by varying the values of the Flow rates to find the optimum Flow rate value to be $0.088 \mathrm{~kg} / \mathrm{s}$. 
Thus, RVAD simulation is carried out at the optimum mesh size and flow rate followed by BiVAD similarly to that of LVAD. Hence, the Bi-VAD which will correspond to the complete design of an artificial pump in constructed and its Efficiency at which it works is found. 


\section{Chapter 5}

\section{Result and Discussion}

\subsection{Introduction}

This Chapter will fundamentally be centering on the mathematical calculations performed to verify whether the design made for the impeller in the previous Chapter can be considered as an ideal design to be implemented and then be used in realistic situations by placing it inside a human body.

The impellers upon being designed in SOLIDWORKS 2018 and then being imported to ANSYS Fluent version 18.1 were simulated and certain crucial parameters' values were extracted from it for calculation and verification purposes. Values of parameters such as pressure, shear stress rate and torque were obtained. Since, certain criterions need to be met for the verification and the accuracy of the impeller being designed such as the value of the Head (represented as ' $\mathrm{H}$ ') of the impeller when converted to $\mathrm{mmHg}$ (i.e., Head pressure) must be in par with the already defined value of it. A healthy human heart has a Head pressure in the range of $100 \mathrm{mmHg}-150 \mathrm{mmHg}$ as defined [1]. The set of calculations will be executed for the three designs of the Ventricular Assist Device (VADs) - LVAD, RVAD and the Bi-VAD and separately the design of LVAD impeller will be run for simulation with different values of blade angle as well as the number of blades along with varying mesh size and number of iterations. The best design of the mesh size with the highest value of Efficiency among all the experimented designs will be chosen to simulate the designs for varying Flow rates from which the best design of the impeller with the highest Efficiency for the most stable mesh size and Flow rate will be chosen to simplify the difficulties of the process and then will be run for the design of impeller of RVAD and further proceeding the Bi-VAD with varying values of Flow rates. The value of the torque and pressure acquired from the simulation will be required to calculate the value of Head, $\mathrm{H}$ and the Head in meters will be converted to the Head pressure in $\mathrm{mmHg}$ which will further be compared to the already stated range of healthy human Head pressure (i.e., $100-150 \mathrm{mmHg}$ ) [1]. If the value of Head calculated falls in that range then it can be evident and stated that the value of Head is 
suitable and that design of impeller can be used in realistic views and thus the verification purpose of the designs is thus met. This value of Head calculated will then be handed down to calculate the value of Efficiency of the impeller.

\subsection{Required equations for fluid flowing mechanism}

In the previous Chapter, Chapter 4 - Design and Simulation it has already been discussed about how the boundary conditions are being applied to the inlet, outlet and even the surrounding walls of the impeller to be built so as to get a sensible perspective on the fluid flowing mechanism taking place within the device. This helps in getting an ultimate best result in the CFD simulation for fluid modeling. As stated already, these parameters can be Mass Flow rate, pressure, velocity which is then applied to the inlet of the impeller during simulation in ANSYS Fluent 18.1 software and depending on that the performance of the outlet will be changing. The mathematical expression in finding out the required output parameters (i.e., Head, $\mathrm{H}$ and Efficiency and even the velocity of the fluid in the inlet and outlet) are stated as below:

The velocity, $\mathrm{V}$ of the fluid flowing through the inlet and then out of the outlet of the impeller can be expressed as [43] :

$$
V=\frac{Q}{A}
$$

Where, $\mathrm{V}=$ Velocity of the fluid flowing through the inlet and out of the outlet of the impeller in $\mathrm{m} / \mathrm{s}$.

$\mathrm{Q}=$ Flow rate of the fluid Flowing along the pathway of the device till the outlet in $\mathrm{m}^{3} /$ seconds.

$\mathrm{A}=$ Area of the inlet of the impeller or the outlet of the impeller in $m^{2}$. The area of the inlet or outlet can be calculated from the diameter, D stated by utilizing the equation as follows [43] :

$$
A=\pi r^{2}
$$


Here,

$\mathrm{r}=$ Radius of the area in $\mathrm{m}$ which is half of the diameter, D. Therefore, equation (5.1.2) can be modified and written as:

$$
A=\pi\left(\frac{D}{2}\right)^{2}
$$

Equation (5.1.3) can further be substituted in equation (5.1.1) to get the final equation of the velocity of the fluid flowing through the inlet and then out of the outlet as follows [43] :

$$
V=\frac{Q}{\pi r^{2}}=\frac{Q}{\pi\left(\frac{D}{2}\right)^{2}}
$$

The Mass Flow rate that is to be applied to the inlet of the impeller as one of the operating conditions during the simulation process in order to acquire the value of the Pressure difference of the fluid Flowing from the inlet and out of the outlet as well as the value of the Torque produced during the rotation of the rotor inside the impeller for the fluid to keep on Flowing along the pathway of the device is represented as [44] :

$$
\text { Mass flow rate }=Q \times \rho
$$

Where,

$\mathrm{Q}=$ Flow rate in $\mathrm{m}^{3} /$ seconds.

$\rho=$ Density of the fluid (i.e., blood having a density of $1056 \mathrm{~kg} / \mathrm{m}^{3}[1]$

. Once the Mass Flow rate is applied to the inlet as a boundary condition, the pressure difference, $\Delta P$ and the torque produced, $\tau$ is acquired upon running the simulation. Taking these values, the calculation for Efficiency and Head of the impeller being designed can be calculated. The Head, $\mathrm{H}$ of the impeller in meters can be calculated as follows with the aid of the mathematical equation [45]:

$$
H e a d=\frac{1}{\rho g}(\Delta P)
$$


To calculate Efficiency of the impeller, the Output power of the impeller as well as the Input power of the impeller is required. The Output power is the hydraulic power of the impeller and the Input power is the mechanical power supplied to the impeller (i.e., power generated during the rotation of the rotor in the impeller for fluid flow movement) and it can be represented in mathematical expression as follows [45] :

$$
\text { Output power }=Q g H \rho
$$

Where, $\mathrm{Q}=$ Flow rate in $\mathrm{m}^{3} /$ seconds.

$\mathrm{g}=$ Acceleration of gravity on the surface of Earth having a value of $9.8 \mathrm{~m} / \mathrm{s}^{2}[1]$. $\mathrm{H}=$ value of Head in meters $\rho=$ Density of the fluid (i.e., blood having a density of $1056 \mathrm{~kg} / \mathrm{m}^{3}$ ) [1].

The input power in terms of a mathematical equation can be represented as follows [45] :

$$
\text { Input power }=\tau \times \omega
$$

Where, $\tau=$ Torque produced during the rotation of the rotor in the impeller in $\mathrm{Nm}$ $\omega=$ Angular frequency of the rotation of the impeller in radians/second (i.e., $\mathrm{rad} / \mathrm{s}$ ).

Thereby, Efficiency of the impeller can be calculated from the output power and input power (equations 5.1.6 and 5.1.7) as shown below [45] :

$$
\text { Efficency }=\frac{\text { Output power }}{\text { Input power }} \times 100 \%=\frac{Q g H \rho}{\tau \times \omega} \times 100 \%
$$

\subsection{Head and Efficiency Calculation of LVAD}

Obtained values of Pressure Difference and Torque upon simulation of the 6 designed impellers of LVAD are used to calculate the Head and Efficiency for 6 blades and 5 blades that are shown separately. 
Starting with the design of the LVAD impeller, the impeller designed following the process as described in Chapter 4 - Design and Simulation, it is simulated for different blade numbers along with varying number of blades and varying mesh size. To account for, the design is simulated for 6 blades, 5 blades with varying blade angles of $100^{\circ}, 110^{\circ}$ and $120^{\circ}$ initially for Mass Flow rate of $0.088 \mathrm{~kg} / \mathrm{s}$ and the angular frequency of rotation of impeller having a value of $400 \mathrm{rpm}$ [1] equivalent to value of $418.67 \mathrm{rad} / \mathrm{s}$ for the LVAD simulation. The table below shows the obtained results from the simulation of LVAD for the most suitable value of mesh size which provides the highest Efficiency. That mesh size which is this case is found out to be $0.3 \mathrm{~mm}$ is taken to simulate all the designs of impellers from which different values of pressure difference and torque is obtained to be further used for calculations of the Head and Efficiency. The table below, Table 5.1 shown is for the following features:

Iteration number: 50

Mass Flow rate: $0.088 \mathrm{~kg} / \mathrm{s}$

\begin{tabular}{|l|l|l|l|l|l|}
\hline Design name & $\begin{array}{l}\text { Mesh } \\
\text { size } \\
(\mathrm{mm})\end{array}$ & Element no. & Node no & $\begin{array}{l}\text { Pressure } \\
\text { difference (Pa) }\end{array}$ & Torque (Nm) \\
\hline Impeller1 & 0.30 & 266070 & 689196 & 17329.095 & 0.00542408 \\
\hline Impeller 2 & 0.30 & 2614791 & 683596 & 17482.5 & 0.00563458 \\
\hline Impeller 3 & 0.30 & 2317530 & 799141 & 19561.539 & 0.00675527 \\
\hline Impeller 4 & 0.30 & 2642436 & 682317 & 16948.29 & 0.005505022 \\
\hline Impeller 5 & 0.30 & 2716706 & 690987 & 22821.422 & 0.00625033 \\
\hline Impeller 6 & 0.30 & 2622578 & 675461 & 19386.52 & 0.155023 \\
\hline
\end{tabular}

Table 5.1 - Obtained Values of Pressure difference, $\Delta P$ and Torque, $\tau$ for all the designed impellers (i.e., Impeller1, 2, 3, 4, 5, 6) run with the best value of mesh size (i.e., $0.30 \mathrm{~mm}$ ) to be used for calculation of Head and Efficiency. 
The values of Head (m) as well as the Efficiency (\%) were obtained by the equations previously stated in this Chapter. Taking Impeller1 which is designed as 6 blades with $100^{\circ}$ wrap angle with a Pressure difference $(\Delta P)$ of $17329.095 \mathrm{~Pa}$ and Torque $(\tau)$ of $0.00542408 \mathrm{Nm}$, the value of Head and Efficiency is obtained by following the steps as shown below:

Initially for LVAD, the Flow rate of $0.3 \mathrm{~m}^{3} /$ hours which is equivalent to $8.33 \times 10^{-5} \mathrm{~m}^{3}$ / seconds. With the aid of the value of Flow rate, the corresponding value of Mass Flow rate required during the simulation of the impeller as an operating condition is calculated from equation (5.1.4) [44] :

$$
\text { Mass flow rate }=Q \times \rho
$$

Since, in this equation the parameter $\mathrm{Q}$ is the Flow rate having a value of $8.33 \times 10^{-5} \mathrm{~m}^{3}$ / seconds and $\rho$ is the density parameter of blood with a value of $1056 \mathrm{~kg} / \mathrm{m}^{3}$ [1] . Substituting in the equation we obtain the value of the Mass Flow rate in $\mathrm{kg} / \mathrm{s}$ as shown [44] :

$$
\text { Mass flow rate }=\left(8.33 \times 10^{-5}\right) \times 1056=0.088 \mathrm{~kg} / \mathrm{s}
$$

Solving this equation gives a Mass Flow rate value of $0.088 \mathrm{~kg} / \mathrm{s}$. The Head, $\mathrm{H}$ in meters is calculated from equation (5.1.5) by substituting the values of the required parameters as follows [45]:

$$
\text { Head, } H=\frac{1}{\rho g}(\Delta P)=\frac{1}{(1056 \times 9.8)}(17329.095)=1.67 \mathrm{~m}
$$

The obtained value of Head of $1.67 \mathrm{~m}$ is furthermore equivalent to $122.82 \mathrm{mmHg}$ which is the considered as the Head pressure for Impeller1. To calculate the Efficiency of Impeller1 being considered the output power along with the input power is required. The output power is calculated by utilizing equation (5.1.6) shown by substituting the value of Head, H previously calculated to be $1.67 \mathrm{~m}$ and other required parameters as shown below [45] : 


$$
\begin{aligned}
\text { Output power } & =Q g H \rho=\left(8.33 \times 10^{-5}\right) \times 9.8 \times 1.67 \times 1056 \\
& =1.44 \text { Watts }
\end{aligned}
$$

Similarly, the input power is calculated from equation (5.1.7) by substituting the values of the Torque and the value of the angular frequency, $\omega$ with a value of $4000 \mathrm{rpm}$ [1] that is equivalent to a value of $418.67 \mathrm{radians} / \mathrm{second}(\mathrm{rad} / \mathrm{s})$ as shown below [45] :

$$
\text { Input power }=\tau \times \omega=0.00542408 \times 418.67=2.27 \text { Watts }
$$

With the obtained values of input power and output power, these values will be substituted in equation (5.1.8) to acquire the value of the Efficiency of the impeller shown below [45] :

$$
\begin{aligned}
\text { Efficency }= & \frac{Q g H \rho}{\tau \times \omega} \times 100 \%=\frac{\left(8.33 \times 10^{-5}\right) \times 9.8 \times 1.67 \times 1056}{0.00542408 \times 418.67} \times 100 \% \\
& =63.44 \%
\end{aligned}
$$

Proceeding to the Impeller2, 3, 4, 5 and 6 the value of Head (m) and Efficiency (\%) will be calculated in the same way as it has been shown for Impeller1 by utilizing the mathematical equations previously stated and shown. Taking Impeller 2 which is designed initially for 5 blades and $100^{\circ}$ wrap angle, the calculation is again carried forward in the same way as it has been done for Impeller1. The value of Head and Efficiency is calculated for Impeller2 with a Pressure difference $(\Delta P)$ of $17482.5 \mathrm{~Pa}$ and Torque $(\tau)$ of $0.00563458 \mathrm{Nm}$ as follows:

The Head, $\mathrm{H}$ in meters is calculated from equation (5.1.5) by substituting the values of the required parameters as follows [45]:

$$
\text { Head, } H=\frac{1}{\rho g}(\Delta P)=\frac{1}{(1056 \times 9.8)}(17482.5)=1.69 \mathrm{~m}
$$

To calculate the Efficiency of Impeller2 being considered the output power along with the input power is required which are calculated as shown below [45] : 


$$
\begin{gathered}
\text { Output power }=Q g H \rho=\left(8.33 \times 10^{-5}\right) \times 9.8 \times 1.69 \times 1056 \\
=1.46 \text { Watts }
\end{gathered}
$$$$
\text { Input power }=\tau \times \omega=0.00563458 \times 418.67=2.36 \text { Watts }
$$

Thus, the Efficiency can be calculated as the ratio of the Output power and the Input power as shown [45] :

$$
\begin{aligned}
\text { Efficency }= & \frac{Q g H \rho}{\tau \times \omega} \times 100 \%=\frac{\left(8.33 \times 10^{-5}\right) \times 9.8 \times 1.69 \times 1056}{0.00563458 \times 418.67} \times 100 \% \\
& =61.89 \%
\end{aligned}
$$

The final table, Table 5.2 with the calculated values of Head and Efficiency for the corresponding Pressure difference and Torque is shown below:

\begin{tabular}{|l|l|l|l|l|l|l|l|l|}
\hline $\begin{array}{l}\text { Design } \\
\text { name }\end{array}$ & $\begin{array}{l}\text { Mesh } \\
\text { size } \\
(\mathrm{mm})\end{array}$ & $\begin{array}{l}\text { Element } \\
\text { no. }\end{array}$ & Node no & $\begin{array}{l}\text { Pressure } \\
\text { difference } \\
(\mathrm{Pa})\end{array}$ & Torque $(\mathrm{Nm})$ & $\begin{array}{l}\text { Head } \\
(\mathrm{m})\end{array}$ & $\begin{array}{l}\text { Head } \\
(\mathrm{mmHg})\end{array}$ & $\begin{array}{l}\text { Efficiency } \\
(\%)\end{array}$ \\
\hline Impeller1 & 0.30 & 266070 & 689196 & 17329.095 & 0.00542408 & 1.67 & 122.81 & 63.44 \\
\hline Impeller 2 & 0.30 & 2614791 & 683596 & 17482.5 & 0.00563458 & 1.69 & 124.27 & 61.89 \\
\hline Impeller 3 & 0.30 & 2317530 & 799141 & 19561.539 & 0.00675527 & 1.89 & 138.95 & 57.60 \\
\hline Impeller 4 & 0.30 & 2642436 & 682317 & 16948.29 & 0.005505022 & 1.64 & 120.60 & 60.78 \\
\hline Impeller 5 & 0.30 & 2716706 & 690987 & 22821.422 & 0.00625033 & 2.21 & 162.52 & 72.90 \\
\hline Impeller 6 & 0.30 & 2622578 & 675461 & 19386.52 & 0.155023 & 1.87 & 137.51 & 2.48 \\
\hline
\end{tabular}

Table 5.2 - The final results table with the calculated values of Head and Efficiency for the corresponding value of Pressure difference and Torque for the Impeller designs with the best mesh size, $0.30 \mathrm{~mm}$. 
Simulation for LVAD with the best design among the six impellers with the combination of the finest Efficiency and suitable value of Head pressure in $\mathrm{mmHg}$ that falls in par with the already stated healthy Head pressure range of $100-150 \mathrm{mmHg}$ [1] for mesh size $0.3 \mathrm{~mm}$ (i.e., Impeller1 designed for $100^{\circ}$ wrap angle and 6 blades gives the combination of the finest Efficiency as well as an Head value of $1.67 \mathrm{~m}$ which when converted to $\mathrm{mmHg}$ gives 122.807 $\mathrm{mmHg}$ that falls within the range of $100-150 \mathrm{mmHg}$ ) [1] has been further carried out for varying Flow rates and corresponding Mass Flow rates values in $\mathrm{kg} / \mathrm{s}$ were used in the simulation process in ANSYS Fluent version 18.1 software and the obtained values of Pressure Difference and Torque for the design of Impeller1 have been used to calculate the Head and Efficiency for the corresponding Mass Flow rates as shown below.

Impeller1 is taken to be the best design among the other designed Impellers since it provides the combination of the appropriate Efficiency of $63.44 \%$ and a Head value of $1.67 \mathrm{~m}$ which proves out to be in par with the realistic values as stated earlier. This Impeller is then run in simulation for varying Flow rates and the output values acquired from performing the simulation are for instance the values of the Pressure difference and Torque which is further used to calculate the values of Head and Efficiency of the impeller. The table, Table 5.3 below shows the corresponding values of Pressure difference and Torque which are obtained for Impeller1 when run under the operating condition of mesh size $0.3 \mathrm{~mm}$ and varying Mass Flow rate values. 


\begin{tabular}{|l|l|l|l|l|}
\hline $\begin{array}{l}\text { Mass Flow } \\
\text { rate }(\mathrm{kg} / \mathrm{s})\end{array}$ & $\begin{array}{l}\text { Mesh size } \\
(\mathrm{mm})\end{array}$ & $\begin{array}{l}\text { Flow rate } \\
\left(\mathrm{m}^{3} / \mathrm{h}\right)\end{array}$ & $\begin{array}{l}\text { Pressure difference } \\
(\mathrm{Pa})\end{array}$ & Torque $(\mathrm{Nm})$ \\
\hline 0.029 & 0.30 & 0.10 & 2793.39 & 0.00593602 \\
\hline 0.044 & 0.30 & 0.15 & 5081.598 & 0.00083534 \\
\hline 0.059 & 0.30 & 0.20 & 8676.593 & 0.00251571 \\
\hline 0.073 & 0.30 & 0.25 & 12115.49 & 0.00499707 \\
\hline 0.088 & 0.30 & 0.30 & 17329.095 & 0.00542408 \\
\hline 0.103 & 0.30 & 0.35 & 22484.4 & 0.00618922 \\
\hline 0.117 & 0.30 & 0.40 & 26760.1 & 0.00989024 \\
\hline 0.132 & 0.30 & 0.45 & 34475.55 & 0.0076888 \\
\hline
\end{tabular}

Table 5.3 - The obtained values of Pressure difference, $\Delta P$ and Torque, $\tau$ for Impellerl being simulated with different Mass Flow rates and the best mesh size of $0.3 \mathrm{~mm}$.

Taking the design of Impellerl with Mass Flow rate value of $0.073 \mathrm{~kg} / \mathrm{s}$ and corresponding obtained values of Pressure difference and Torque from simulation, the Head (m) and Efficiency (\%) can be calculated as follows from the previously stated equations as follows:

Pressure difference $(\Delta P)$ obtained for Impeller1 with Mass Flow rate of $0.073 \mathrm{~kg} / \mathrm{s}$ for flow rate of $0.25 \mathrm{~m}^{3} / \mathrm{h}$ is obtained to be $12115.49 \mathrm{~Pa}$ and Torque value $(\tau)$ of $0.00499707 \mathrm{Nm}$. The value of angular frequency of the rotation of the impeller remains the same as before as $4000 \mathrm{rpm}$ [1] which is equivalent to value of $418.67 \mathrm{rad} / \mathrm{s}$ since $4000 \mathrm{rpm}$ is used for the simulation of LVAD [1].

The Head, $\mathrm{H}$ in meters is calculated from equation (5.1.5) by substituting the values of the required parameters as follows [45] :

$$
\text { Head, } H=\frac{1}{\rho g}(\Delta P)=\frac{1}{(1056 \times 9.8)}(12115.49)=1.17 \mathrm{~m}
$$


The Efficiency of the impeller is further calculated from the ratio of the Output power of the impeller and the Input power of the impeller. Separately the Input power and Output power can be calculated as shown below. The Output power is calculated from equation (5.1.6) and Input power is calculated from equation (5.1.7).

The value of Mass Flow rate is now $0.073 \mathrm{~kg} / \mathrm{s}$ (i.e., Flow rate, $\mathrm{Q}=0.25 \mathrm{~m}^{3} / \mathrm{h}$ ) which previously is $0.088 \mathrm{~kg} / \mathrm{s}$ (i.e., Flow rate, $\mathrm{Q}=0.30 \mathrm{~m}^{3} / \mathrm{h}$ ) and used for calculating the value of Head and Efficiency. Using Mass Flow rate of $0.073 \mathrm{~kg} / \mathrm{s}$, the Output power is calculated as shown:

Since the corresponding value of Flow rate for Mass Flow rate of $0.073 \mathrm{~kg} / \mathrm{s}$ can be calculated from equation (5.1.4) as follows [44] :

$$
\text { Mass flow rate }=Q \times \rho
$$

This equation gives a Flow rate value of $6.94 \times 10^{-5} \mathrm{~m}^{3} / \mathrm{s}$. Thus, using the value of Mass Flow rate and substituting in equation (5.1.6), the Output power can be calculated as follows [45] :

$$
\begin{aligned}
\text { Output power } & =Q g H \rho=\left(6.94 \times 10^{-5}\right) \times 9.8 \times 1.17 \times 1056 \\
& =0.840 \text { Watts }
\end{aligned}
$$

The input power is calculated as follows from equation (5.1.7) [45] :

$$
\text { Input power }=\tau \times \omega=0.00499707 \times 418.67=2.09 \text { Watts }
$$

Thus, Efficiency of the impeller is calculated as the ratio of the Output power to the Input Power as shown [45]:

$$
\begin{aligned}
\text { Efficency }= & \frac{Q g H \rho}{\tau \times \omega} \times 100 \%=\frac{\left(6.92 \times 10^{-5}\right) \times 9.8 \times 1.17 \times 1056}{0.00499707 \times 418.67} \times 100 \% \\
& =40.19 \%
\end{aligned}
$$


The calculations of the Head and Efficiency, taking into consideration the other values of Mass Flow rates with the aid of the values of Pressure difference and Torque obtained from simulation under the operating condition of the corresponding value of Mass Flow rate can thus be calculated in the same way that it has been done for Mass Flow rate value of $0.073 \mathrm{~kg} / \mathrm{s}$. Thus, the final table with the calculated values of Head and Efficiency for the corresponding values of Mass Flow rates and Pressure difference and Torque for Impeller 1 designed as $100^{\circ}$ wrap angle and 6 blades is shown below in Table 5.4 .

\begin{tabular}{|l|l|l|l|l|l|l|}
\hline $\begin{array}{l}\text { Mass } \\
\text { Flow rate } \\
(\mathrm{kg} / \mathrm{s})\end{array}$ & $\begin{array}{l}\text { Mesh } \\
\text { size } \\
(\mathrm{mm})\end{array}$ & $\begin{array}{l}\text { Flow } \\
\text { rate } \\
\left(\mathrm{m}^{3} / \mathrm{h}\right)\end{array}$ & $\begin{array}{l}\text { Pressure } \\
\text { difference } \\
(\mathrm{Pa})\end{array}$ & Torque $(\mathrm{Nm})$ & Head $(\mathrm{m})$ & $\begin{array}{l}\text { Efficiency } \\
(\%)\end{array}$ \\
\hline 0.029 & 0.30 & 0.10 & 2793.39 & 0.00593602 & 0.270 & 3.13 \\
\hline 0.044 & 0.30 & 0.15 & 5081.598 & 0.00083534 & 0.491 & 60.57 \\
\hline 0.059 & 0.30 & 0.20 & 8676.593 & 0.00251571 & 0.838 & 45.77 \\
\hline 0.073 & 0.30 & 0.25 & 12115.49 & 0.00499707 & 1.17 & 40.19 \\
\hline 0.088 & 0.30 & 0.30 & 17329.095 & 0.00542408 & 1.67 & 63.44 \\
\hline 0.103 & 0.30 & 0.35 & 22484.4 & 0.00618922 & 2.17 & 84.17 \\
\hline 0.117 & 0.30 & 0.40 & 26760.1 & 0.00989024 & 2.59 & 71.98 \\
\hline 0.132 & 0.30 & 0.45 & 34475.55 & 0.0076888 & 3.33 & 133.85 \\
\hline
\end{tabular}

Table 5.4 - The calculated values of Head and Efficiency for the Pressure difference and Torque for Impeller1 being simulated for different Mass Flow rates and mesh size of $0.3 \mathrm{~mm}$

\subsection{Graphical representations for comparison of LVAD Impellers}

In order to reach the final conclusion of which Impeller design among the designed Impellers (i.e., Impeller1, 2, 3, 4, 5 and 6) with the best mesh size and the best flow rate that gives an acceptable combination of high Efficiency and Head which when converted into Head pressure stays in par with the already defined healthy human Head pressure that has already been stated earlier in this Chapter and can be considered as the best one for the LVAD to be used for the 
simulation of RVAD and Bi-VAD, thus to provide reasoning on which Impeller should be considered along with the calculated values of Head and Efficiency as shown earlier, the graphical representations comes in handy since they provide a vivid, clear idea and a better comparison method to emphasize on the decision of the best Impeller of LVAD to be chosen for the rest simulation of RVAD and Bi-VAD. The following graphs are shown below for the 6 designed Impellers for the LVAD that has been already discussed about in Chapter 4.

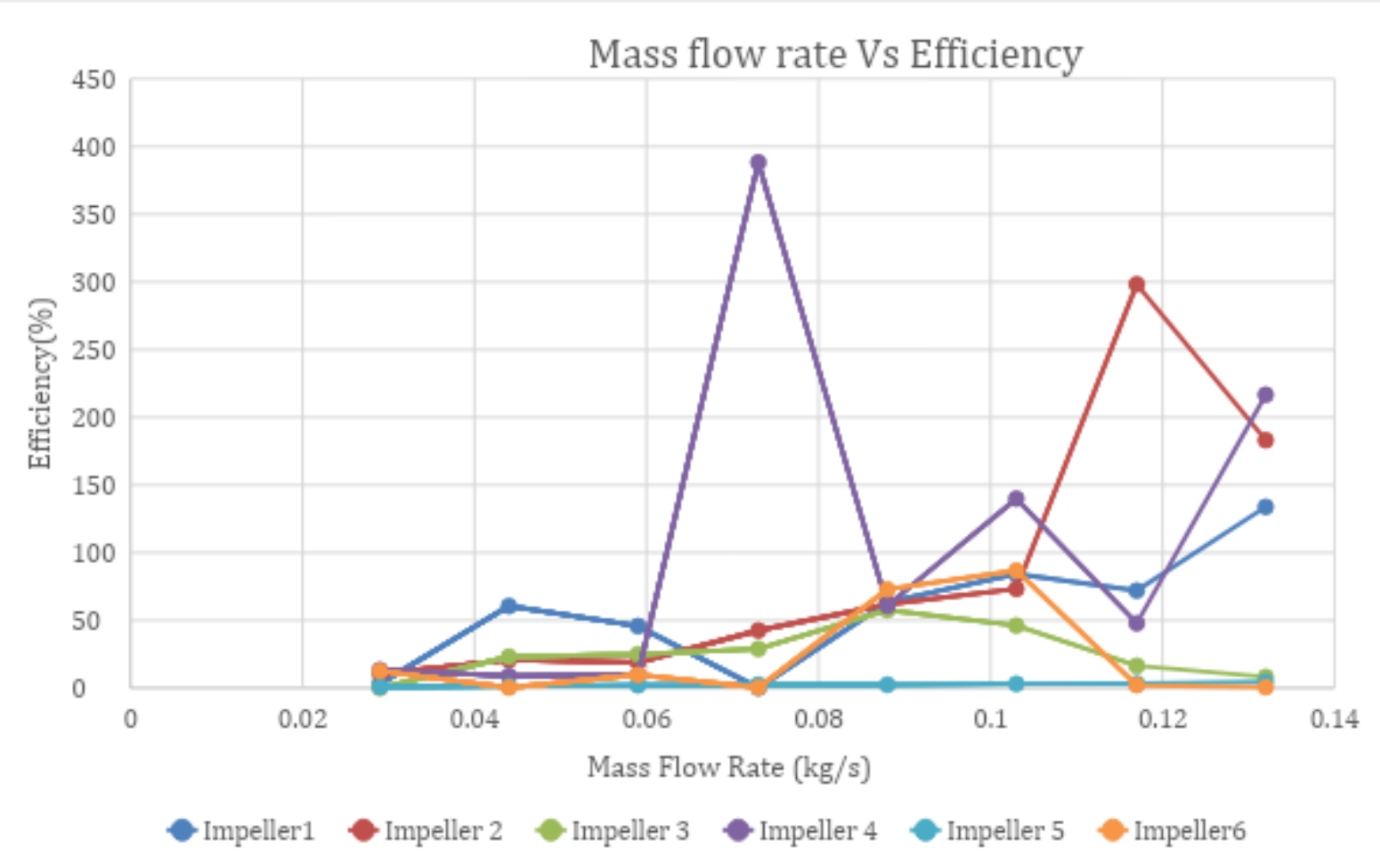

Figure 5.1 - The above diagram illustrates the visual representation of the Mass Flow rates Vs Efficiency for all the designed Impellers for LVAD (i.e., Impeller1, 2, 3, 4, 5 and 6). 


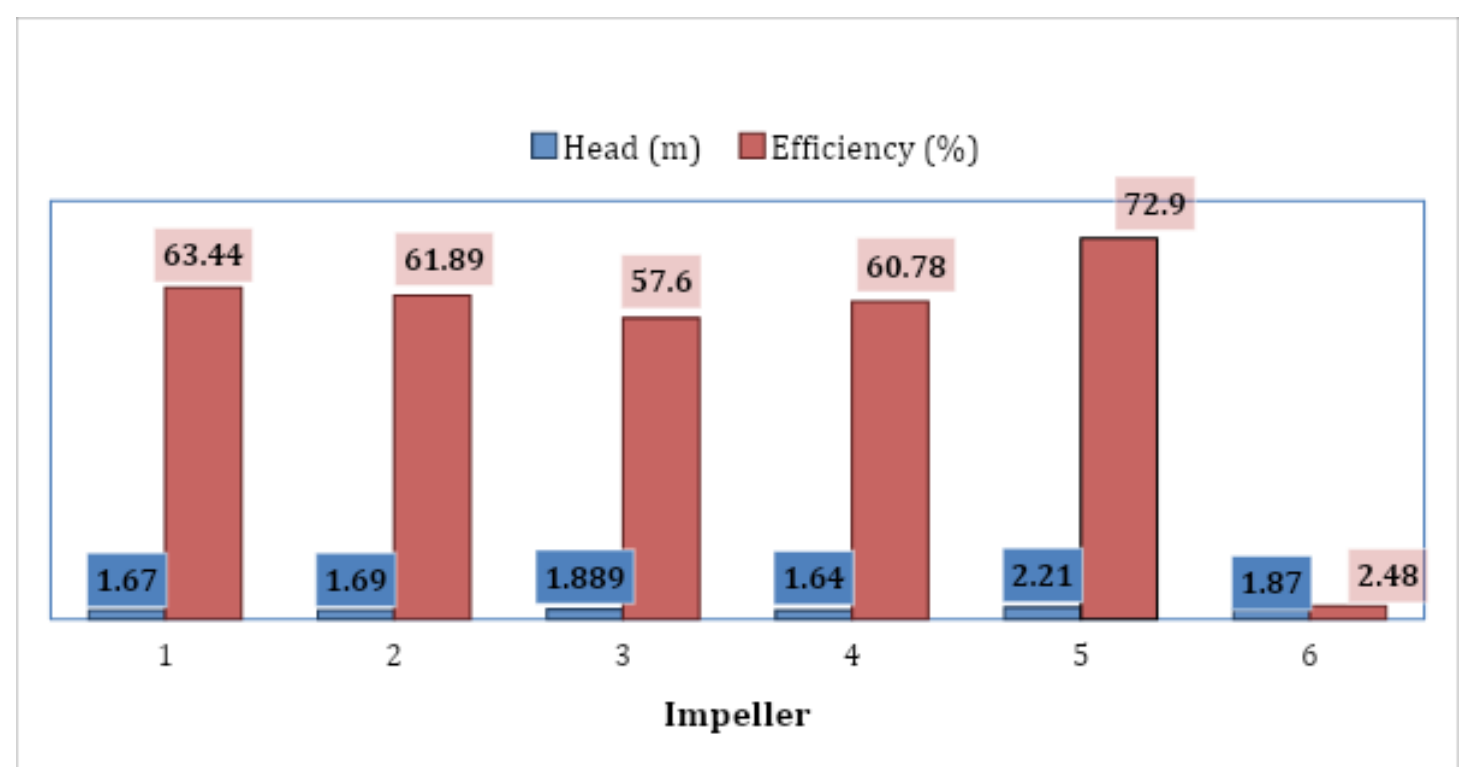

Figure 5.2 - The above graph illustrates the bar graph representation of Head and corresponding Efficiency for all the 6 designed Impellers of LVAD (i.e., Impeller1, 2, 3, 4, 5 and 6) at a mesh size of $0.3 \mathrm{~mm}$.

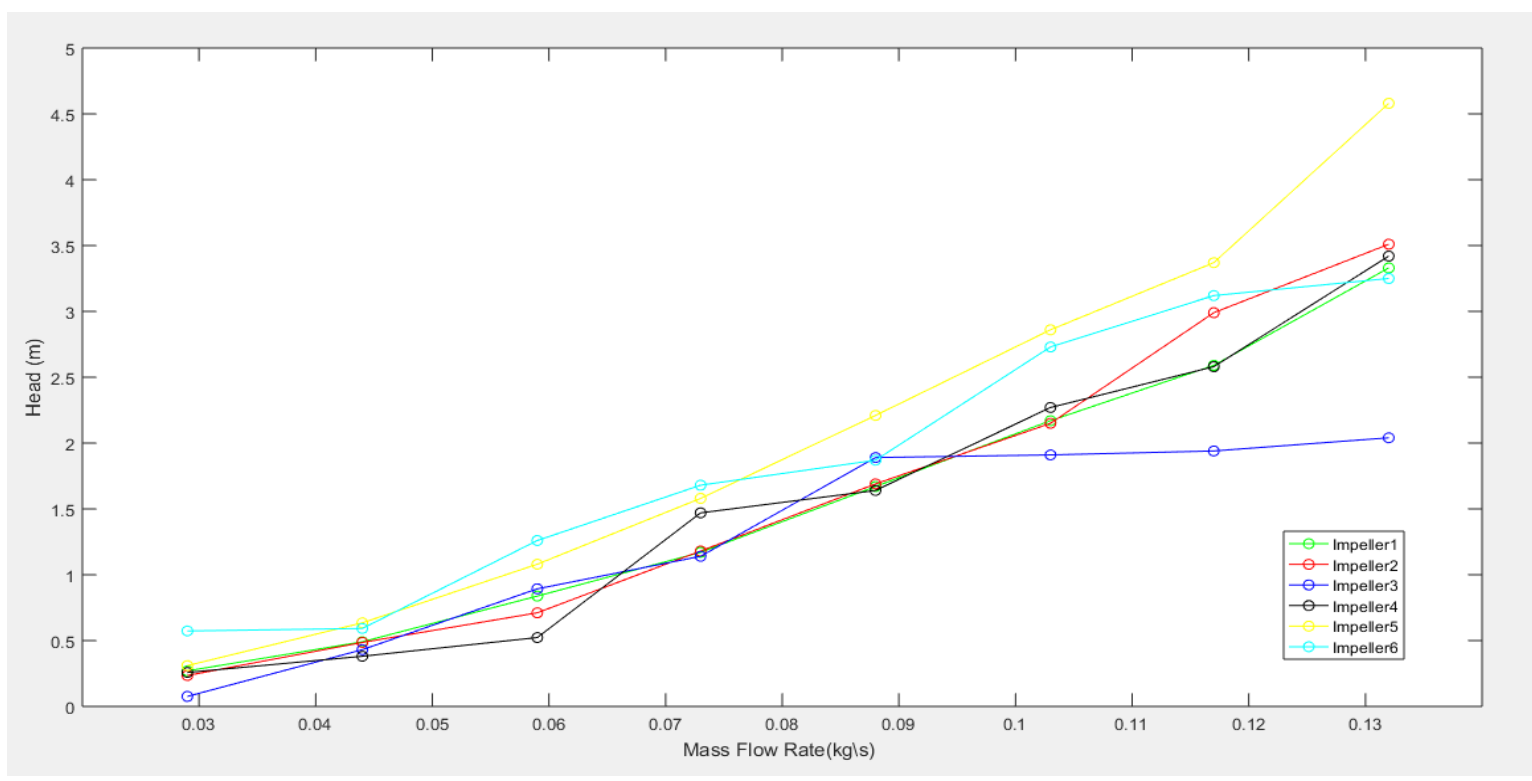

Figure 5.3 - The graph illustrates the graphical form of the Head Vs Mass Flow rate of the 6 designed Impellers of LVAD (i.e., Impeller1, 2, 3, 4, 5, 6). 


\subsection{Conclusion on ultimate best Impeller design of LVAD:}

From the calculations previously done in this Chapter and the graphical representations shown above for all the designed impellers of the LVAD, the final decision of considering which impeller will be considered as the ultimate best model for the single sided LVAD to be handed down for the simulation of the single sided RVAD and the Bi-VAD. From Figure 5.2, this is the bar graph representation of Head and corresponding Efficiency for all the 6 designed impellers for the LVAD. The bar graph provided a clear, transparent view that Impellerl provides the finest combination of both a suitable Head value which when converted to Head pressure stays in par with the already defined range of healthy Head pressure (i.e., $100-150 \mathrm{mmHg}$ ) [1] when simulated for a mesh size of $0.3 \mathrm{~mm}$ and also a appropriate value of Efficiency of $63.44 \%$. However, since all the impellers gave a considerable and appropriate value of Head as well as Efficiency at the mesh size of $0.3 \mathrm{~mm}$. Hence this mesh size is considered as the best mesh size amongst all and at this value of mesh size; all the 6 designed impellers for the LVAD were then simulated with varying Mass Flow rates and from the graphical representation of the Head Vs Mass Flow rates shown in Figure 5.3, it shows a vivid, clear idea of how at a Flow rate value of $0.3 \mathrm{~m}^{3} / \mathrm{h}$ that is equivalent to a Mass Flow rate value of $0.088 \mathrm{~kg} / \mathrm{s}$, all the impellers considered in total showed a perfect, considerable value of Head (m) and amongst all the 6 designed impellers, Impeller1 has the perfect and highest value of Head to be considered at this Mass Flow rate value of $0.088 \mathrm{~kg} / \mathrm{s}$. Furthermore, the graphical representation of the Efficiency Vs Mass Flow rates shown in Figure 5.1 clearly shows that at a Mass Flow rate value of $0.088 \mathrm{~kg} / \mathrm{s}$ (i.e., Flow rate, $\mathrm{Q}=0.3 \mathrm{~m}^{3} / \mathrm{h}$ ), all the designed impellers shows the finest value of Efficiency. Even though Impeller1 which is more dominant than the other impellers in the previous two scenarios, however in Figure 5.1, even though at the Mass Flow rate value of $0.088 \mathrm{~kg} / \mathrm{s}$, Impeller1 did not have the highest Efficiency but when combined with its Head value at that Mass Flow rate shown in Figure 5.3, both the values of Efficiency and the Head are the finest values to be considered. Therefore, Impeller1 can be used as the ideal model of the best impeller design of LVAD to be considered for the RVAD and the Bi-VAD. In figure 5.1, it is observed that at some Mass Flow rate values, some impellers have values of Efficiency above 100\% which is quite unacceptable. For this reason, these values are opted out of consideration, example of which is Impeller4 which has a sharp change in the value of the Efficiency near the value of 
Mass Flow rate of $0.08 \mathrm{~kg} / \mathrm{s}$, the value of the Efficiency goes higher than $100 \%$, even higher than $300 \%$ which makes it to be an unacceptable value of Efficiency of an impeller since the value of the Efficiency is not an ideal one and cannot be utilized. On top of that, in [1], it has been stated of how Impeller 2 in that case designed for 6 blades and $110^{\circ}$ wrap angle showed the finest results of the highest Head value as well as the highest Efficiency value amongst all the other designed impellers and thereby it has been considered as the ultimate best design. However, as it has been shown in Chapter 4, Impeller1 has been designed for 6 blades and $100^{\circ}$ wrap angle and later when simulated and with the obtained results from simulation the calculations were done in Chapter 5 from where it has been seen that this Impeller1 obtained the finest combination of the value of Head and the value of Efficiency with a value of $63.44 \%$ which is higher than the value of Efficiency of Impeller2 as considered in [1] with a value of Efficiency of less than $60 \%$. Thereby, it can be concluded that if the Impeller2 being considered in [1] is further modified by keeping the same number of blades, i.e., 6 but by changing the value of the wrap angle from $110^{\circ}$ to $100^{\circ}$ the value of the Efficiency can further be increased to a higher value than it was achieved in [1]. It is quite evident now that Impeller1 designed for 6 blades and $100^{\circ}$ wrap angle for LVAD is dominant over the other impellers with different blade numbers and even wrap angles. Furthermore, Impeller1 has the best characteristics when compared to the rest 5 impellers (i.e., Impeller2, 3, 4, 5 and 6), leading to the reason why it will be considered as the perfect ideal model of the LVAD and then be handed - down for the simulation of the RVAD and LVAD under the same characteristics as the best chosen model (i.e., Impeller1 with a mesh size of 0.3 $\mathrm{mm}$ and Mass Flow rate value of $0.088 \mathrm{~kg} / \mathrm{s}$ ).

\subsection{Head and Efficiency Calculation of RVAD}

The simulation of the RVAD has been done with the best-chosen design of impeller in LVAD that is Impeller1 simulated under the operating conditions of mesh size of $0.3 \mathrm{~mm}$ and Flow rate of $0.3 \mathrm{~m}^{3} / \mathrm{h}$. Along with these operating conditions the RVAD has been simulated with an angular frequency of rotation of impeller having a value of $3054 \mathrm{rpm}$ [1] which in the case of LVAD is $4000 \mathrm{rpm}[1]$ and thus when simulated the values of Pressure difference $(\Delta P)$ and Torque $(\tau)$ were obtained as such shown in Table 5.5 


\begin{tabular}{|l|l|l|l|l|}
\hline $\begin{array}{l}\text { Mass Flow } \\
\text { rate }(\mathrm{kg} / \mathrm{s})\end{array}$ & $\begin{array}{l}\text { Mesh size } \\
(\mathrm{mm})\end{array}$ & $\begin{array}{l}\text { Flow rate } \\
\left(\mathrm{m}^{3} / \mathrm{h}\right)\end{array}$ & $\begin{array}{l}\text { Pressure difference } \\
(\mathrm{Pa})\end{array}$ & Torque (Nm) \\
\hline 0.088 & 0.30 & 0.30 & 16427.8 & 0.00503789 \\
\hline
\end{tabular}

Table 5.5 - Obtained values of Pressure difference, $\Delta P$ and Torque, $\tau$ for RVAD upon simulation with conditions of $0.3 \mathrm{~mm}$ mesh size, $0.3 \mathrm{~m}^{3} / \mathrm{h}$ Flow rate and an angular frequency of rotation having a value of $3054 \mathrm{rpm}[1]$.

For the ease of calculating the values of the Head and Efficiency of the impeller, the same mathematical equations that has been used earlier for calculation purposes for LVAD is used in the case of RVAD as well as shown below:

Initially, the Flow rate of $0.3 \mathrm{~m}^{3}$ /hour that is equivalent to $8.33 \times 10^{-5} \mathrm{~m}^{3} / \mathrm{seconds}$ is being utilized during the calculation of the Mass Flow rate for the impeller. From the value of the Flow rate, the Mass Flow rate required for the simulation is calculated from equation (5.1.4) [44] :

$$
\text { Mass flow rate }=Q \times \rho
$$

Since, in this equation the parameter $\mathrm{Q}$ is the Flow rate having a value of $8.33 \times 10^{-5} \mathrm{~m}^{3}$ / seconds and $\rho$ is the density parameter of blood with a value of $1056 \mathrm{~kg} / \mathrm{m}^{3}$ [1]. Substituting in the equation value of the Mass Flow rate in $\mathrm{kg} / \mathrm{s}$ can be obtained as shown [44] :

$$
\text { Mass flow rate }=\left(8.33 \times 10^{-5}\right) \times 1056=0.088 \mathrm{~kg} / \mathrm{s}
$$

For the corresponding value of Flow rate of $0.3 \mathrm{~m}^{3}$ /hours, the value of the obtained Mass Flow rate is $0.088 \mathrm{~kg} / \mathrm{s}$. The Head, $\mathrm{H}$ in meters is calculated from equation (5.1.5) by substituting the values of the required parameters as follows. For this instance, the value of the Pressure difference $(\Delta P)$ is the obtained Pressure difference upon simulation for the RVAD. The Head is calculated as follows [45]: 


$$
\text { Head, } H=\frac{1}{\rho g}(\Delta P)=\frac{1}{(1056 \times 9.8)}(16427.8)=1.59 \mathrm{~m}
$$

The obtained value of Head is thus calculated to have a value of $1.65 \mathrm{~m}$. To calculate the Efficiency, the output power along with the input power is required. The output power is calculated by utilizing equation (5.1.6) shown by substituting the value of Head, H previously calculated to be $1.67 \mathrm{~m}$ and other required parameters as shown below [45] :

$$
\begin{aligned}
\text { Output power } & =Q g H \rho=\left(8.33 \times 10^{-5}\right) \times 9.8 \times 1.59 \times 1056 \\
& =1.37 \text { Watts }
\end{aligned}
$$

Similarly, the input power is calculated from equation (5.1.7) by substituting the values of the Torque and the value of the angular frequency, $\omega$ with a value of $3054 \mathrm{rpm}$ [1] that is equivalent to a value of $319.652 \mathrm{radians} / \mathrm{second}(\mathrm{rad} / \mathrm{s})$ as shown below [45] :

$$
\text { Input power }=\tau \times \omega=0.00503789 \times 319.652=1.61 \text { Watts }
$$

With the obtained values of input power and output power, these values will be substituted in equation (5.1.8) to acquire the value of the Efficiency of the impeller shown below [45] :

$$
\begin{aligned}
\text { Efficency }= & \frac{Q g H \rho}{\tau \times \omega} \times 100 \%=\frac{\left(8.33 \times 10^{-5}\right) \times 9.8 \times 1.59 \times 1056}{0.00503789 \times 319.652} \times 100 \% \\
& =85.09 \%
\end{aligned}
$$

Thus, the final table consisting of the calculated Head and Efficiency for the corresponding value of Pressure difference and Torque for RVAD in Table 5.6 


\begin{tabular}{|l|l|l|l|l|l|l|}
\hline $\begin{array}{l}\text { Mass } \\
\text { Flow } \\
\text { rate } \\
(\mathrm{kg} / \mathrm{s})\end{array}$ & $\begin{array}{l}\text { Mesh } \\
\text { size } \\
(\mathrm{mm})\end{array}$ & $\begin{array}{l}\text { Flow rate } \\
\left(\mathrm{m}^{3} / \mathrm{h}\right)\end{array}$ & $\begin{array}{l}\text { Pressure } \\
\text { difference } \\
(\mathrm{Pa})\end{array}$ & Torque $(\mathrm{Nm})$ & Head $(\mathrm{m})$ & $\begin{array}{l}\text { Efficiency } \\
(\%)\end{array}$ \\
\hline 0.088 & 0.30 & 0.30 & 16427.8 & 0.00503789 & 1.59 & 85.09 \\
\hline
\end{tabular}

Table 5.6 - The calculated values of Head and Efficiency for the corresponding values of Pressure difference and Torque for RVAD with the operating conditions for RVAD described earlier.

\subsection{Head and Efficiency calculation of Bi-VAD}

For the simulation of the Bi-VAD, no exceptional method has been opted for except for the LVAD best design and the simulated RVAD being conjoined together as talked about previously in Chapter 4 - Design and Simulation. Before conjoining, separately for the single sided LVAD and RVAD the values of the Head and Efficiency previously calculated in this Chapter are shown below in tabular form in Table 5.7(a) and (b).

\begin{tabular}{|l|l|l|l|l|l|l|}
\hline $\begin{array}{l}\text { Mass } \\
\text { Flow } \\
\text { rate } \\
(\mathrm{kg} / \mathrm{s})\end{array}$ & $\begin{array}{l}\text { Mesh } \\
\text { size } \\
(\mathrm{mm})\end{array}$ & $\begin{array}{l}\text { Flow rate } \\
\left(\mathrm{m}^{3} / \mathrm{h}\right)\end{array}$ & $\begin{array}{l}\text { Pressure } \\
\text { difference } \\
(\mathrm{Pa})\end{array}$ & Torque $(\mathrm{Nm})$ & Head $(\mathrm{m})$ & $\begin{array}{l}\text { Efficiency } \\
(\%)\end{array}$ \\
\hline 0.088 & 0.30 & 0.30 & 17329.095 & 0.00542408 & 1.67 & 63.44 \\
\hline
\end{tabular}

Table 5.7(a) - The calculated values of Head and Efficiency for the corresponding Pressure difference and Torque for the best design of LVAD under operating conditions of $0.30 \mathrm{~m}^{3} / \mathrm{h}$ Flow rate, mesh size of $0.30 \mathrm{~mm}$, Mass Flow rate of $0.088 \mathrm{~kg} / \mathrm{s}$ and an angular speed of rotation of impeller having a value of $4000 \mathrm{rpm}$ [1] before being conjoined together 


\begin{tabular}{|l|l|l|l|l|l|l|}
\hline $\begin{array}{l}\text { Mass } \\
\text { Flow } \\
\text { rate } \\
(\mathrm{kg} / \mathrm{s})\end{array}$ & $\begin{array}{l}\text { Mesh } \\
\text { size } \\
(\mathrm{mm})\end{array}$ & $\begin{array}{l}\text { Flow rate } \\
\left(\mathrm{m}^{3} / \mathrm{h}\right)\end{array}$ & $\begin{array}{l}\text { Pressure } \\
\text { difference } \\
(\mathrm{Pa})\end{array}$ & Torque $(\mathrm{Nm})$ & Head $(\mathrm{m})$ & $\begin{array}{l}\text { Efficiency } \\
(\%)\end{array}$ \\
\hline 0.088 & 0.30 & 0.30 & 16427.8 & 0.00503789 & 1.59 & 85.09 \\
\hline
\end{tabular}

Table 5.7(b) - The calculated values of Head and Efficiency for the corresponding Pressure difference and Torque for the best design of RVAD simulated with the best design of LVAD under operating conditions of $0.30 \mathrm{~m}^{3} / \mathrm{h}$ Flow rate, mesh size of $0.30 \mathrm{~mm}$, Mass Flow rate of $0.088 \mathrm{~kg} / \mathrm{s}$ and angular speed of rotation of impeller having a value of $3054 \mathrm{rpm}$ [1] before being conjoined together.

After being joined together (i.e., LVAD and RVAD) to form the $\mathrm{Bi}$ - VAD, there has been certain changes in the Pressure difference and Torque of both the LVAD and RVAD separately upon simulation that has been shown in Table 5.8

\begin{tabular}{|l|l|l|l|l|l|}
\hline $\begin{array}{l}\text { Single } \\
\text { sided } \\
\text { VAD }\end{array}$ & $\begin{array}{l}\text { Mass Flow } \\
\text { rate }(\mathrm{kg} / \mathrm{s})\end{array}$ & $\begin{array}{l}\text { Mesh size } \\
(\mathrm{mm})\end{array}$ & $\begin{array}{l}\text { Flow rate } \\
\left(\mathrm{m}^{3} / \mathrm{h}\right)\end{array}$ & $\begin{array}{l}\text { Pressure difference } \\
(\mathrm{Pa})\end{array}$ & Torque (Nm) \\
\hline LVAD & 0.088 & 0.30 & 0.30 & 10371.37 & 0.00355263 \\
\hline RVAD & 0.088 & 0.30 & 0.30 & 12796.53 & 0.00630962 \\
\hline
\end{tabular}

Table 5.8 - The obtained Pressure difference, $\Delta P$ and Torque, $\tau$ for LVAD and RVAD separately upon simulation of Bi-VAD.

The values of Head and Efficiency for the corresponding values of Pressure difference and Torque for RVAD and LVAD are calculated in the same way as before with the mathematical equations stated earlier. 
For LVAD the values of Head and Efficiency are calculated as show below. The Head, $\mathrm{H}$ is calculated as follows [45] :

$$
\text { Head, } H=\frac{1}{\rho g}(\Delta P)=\frac{1}{(1056 \times 9.8)}(10371.37)=1.00 \mathrm{~m}
$$

The above calculation brings out the value of Head in meters to be $1.00 \mathrm{~m}$. To calculate the Efficiency, the output power along with the input power is required. The output power is calculated by utilizing equation (5.1.6) shown by substituting the value of Head, $\mathrm{H}$ previously calculated to be $1.67 \mathrm{~m}$ and other required parameters as shown below. The value of Q, Flow rate in this case is $0.3 \mathrm{~m}^{3} / \mathrm{h}$ that is equivalent to $8.33 \times 10^{-5} \mathrm{~m}^{3} /$ seconds. The output power is calculated as follows [45] :

$$
\begin{aligned}
\text { Output power } & =Q g H \rho=\left(8.33 \times 10^{-5}\right) \times 9.8 \times 1.00 \times 1056 \\
& =0.862 \mathrm{Watts}
\end{aligned}
$$

Similarly, the input power is calculated from equation (5.1.7) by substituting the values of the Torque and the value of the angular frequency, $\omega$ with a value of $4000 \mathrm{rpm}$ [1] in the case of LVAD that is equivalent to a value of 418.67 radians/second ( $\mathrm{rad} / \mathrm{s})$ as shown below [45] :

$$
\text { Input power }=\tau \times \omega=0.00355263 \times 418.67=1.49 \text { Watts }
$$

With the obtained values of input power and output power, these values will be substituted in equation (5.1.8) to acquire the value of the Efficiency as shown below [45] :

$$
\begin{aligned}
\text { Efficency }= & \frac{Q g H \rho}{\tau \times \omega} \times 100 \%=\frac{\left(8.33 \times 10^{-5}\right) \times 9.8 \times 1.00 \times 1056}{0.00355263 \times 418.67} \times 100 \% \\
& =57.85 \%
\end{aligned}
$$

Like the LVAD, similarly the value of Head and Efficiency for the corresponding values of Pressure difference and Torque obtained upon simulation for the RVAD can be calculated as follows. The value of Head is calculated as follows [45] : 


$$
\text { Head, } H=\frac{1}{\rho g}(\Delta P)=\frac{1}{(1056 \times 9.8)}(12796.53)=1.24 \mathrm{~m}
$$

The output power is calculated by utilizing equation (5.1.6) shown by substituting the value of Head, $\mathrm{H}$ previously calculated to be $1.67 \mathrm{~m}$ and other required parameters as shown below [45] :

$$
\begin{gathered}
\text { Output power }=Q g H \rho=\left(8.33 \times 10^{-5}\right) \times 9.8 \times 1.24 \times 1056 \\
=1.07 \text { Watts }
\end{gathered}
$$

Similarly, the input power is calculated from equation (5.1.7) by substituting the values of the Torque and the value of the angular frequency, $\omega$ of the rotation of the impeller for RVAD has a value of $3054 \mathrm{rpm}$ [1], equivalent to a value of $319.652 \mathrm{radians} / \mathrm{second}(\mathrm{rad} / \mathrm{s})$ which is then implemented in the equation for calculation as shown below [45] :

$$
\text { Input power }=\tau \times \omega=0.00630962 \times 319.652=2.02 \text { Watts }
$$

With the obtained values of input power and output power, value of the Efficiency of RVAD is calculated as [45]:

$$
\begin{aligned}
\text { Efficency }= & \frac{Q g H \rho}{\tau \times \omega} \times 100 \%=\frac{\left(8.33 \times 10^{-5}\right) \times 9.8 \times 1.24 \times 1056}{0.00630962 \times 319.652} \times 100 \% \\
& =52.97 \%
\end{aligned}
$$

The final table along with the calculated values of Head and Efficiency for the corresponding values of Pressure difference and Torque for the LVAD and RVAD separately that has been obtained upon simulation of Bi-VAD conjoining the LVAD and RVAD together as shown in Table 5.9 


\begin{tabular}{|c|c|c|c|c|c|c|c|}
\hline $\begin{array}{l}\text { Single } \\
\text { sided } \\
\text { VAD }\end{array}$ & $\begin{array}{l}\text { Mass } \\
\text { Flow } \\
\text { rate } \\
(\mathrm{kg} / \mathrm{s})\end{array}$ & $\begin{array}{l}\text { Mesh } \\
\text { size } \\
(\mathrm{mm})\end{array}$ & $\begin{array}{l}\text { Flow } \\
\text { rate } \\
\left(\mathrm{m}^{3} / \mathrm{h}\right)\end{array}$ & $\begin{array}{l}\text { Pressure } \\
\text { difference } \\
(\mathrm{Pa})\end{array}$ & Torque $(\mathrm{Nm})$ & $\begin{array}{l}\text { Head } \\
\text { (m) }\end{array}$ & $\begin{array}{l}\text { Efficiency } \\
(\%)\end{array}$ \\
\hline LVAD & 0.088 & 0.30 & 0.30 & 10371.37 & 0.00355263 & 1.00 & 57.85 \\
\hline RVAD & 0.088 & 0.30 & 0.30 & 12796.53 & 0.00630962 & 1.24 & 52.97 \\
\hline
\end{tabular}

Table 5.9 - The calculated values of Head and Efficiency for the corresponding values of Pressure difference and Torque for LVAD and RVAD upon simulation of Bi-VAD.

Therefore, the value of Efficiency of the Bi-VAD can be calculated as the average of the Efficiency of the LVAD and the Efficiency of the RVAD after conjoining them together which gives the Efficiency of Bi-VAD to be $55.41 \%$.

\subsection{Hemolysis Parameters Analysis}

On the purpose of reviewing the performance of the pump the vital hemolysis parameters for instance, the pressure distribution, velocity and shear stress profile contours for each of the design at an operating condition of Mass Flow rate having a value of $0.088 \mathrm{~kg} / \mathrm{s}$ are shown in the Figures (5.4-5.9). Impellers with 6 blades (i.e., Impeller1, 3 and 5) have higher pressure distribution than impellers with 5 blades matching our simulation results. However, Impeller6 has very high pressure throughout the volute casing, which makes it unacceptable as a pump. Almost all the designs of the impellers have high velocity at the impeller edges as shown in the velocity contours. Impeller2, 5 and 6 has high shear regions around the blades. Impeller1, 3 and 4 has low shear regions throughout the impeller portion. The impact of the shear stress should be further analyzed to see whether it affects the RBC (Red Blood Cells) or not [46]. 


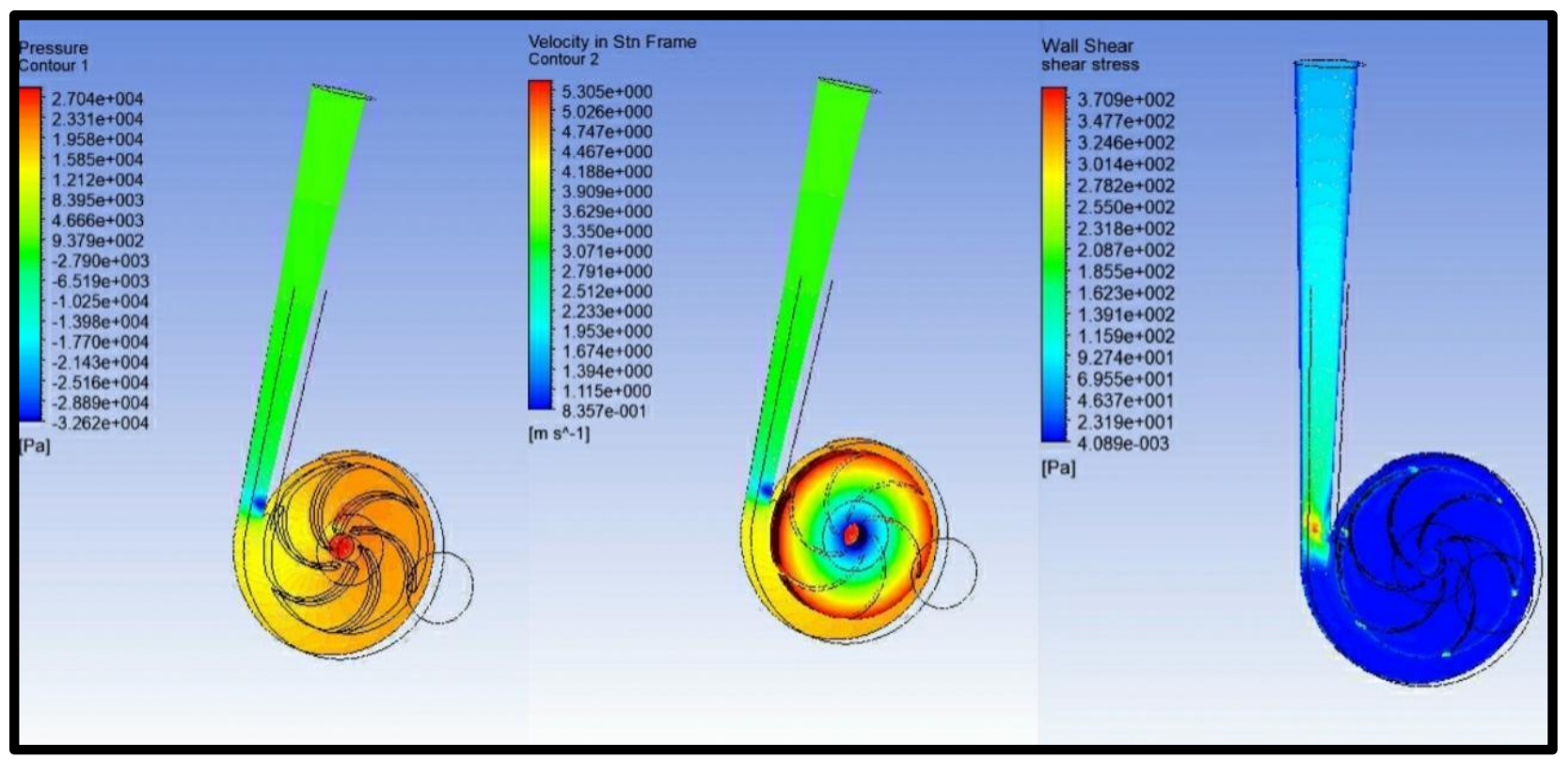

Figure 5.4: Impeller 1 pressure, velocity and shear stress profile

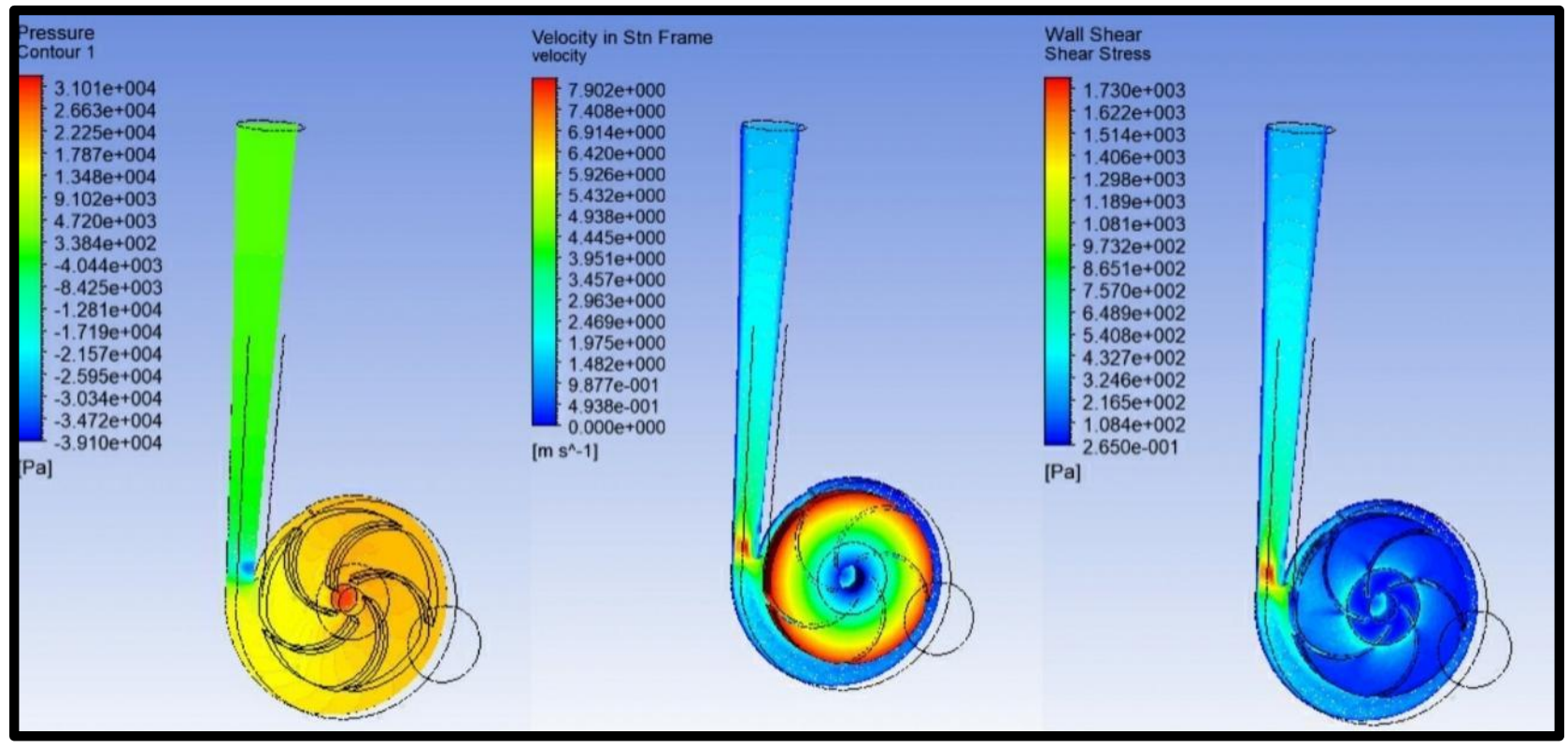

Figure 5.5: Impeller 2 pressure, velocity and shear stress profile 


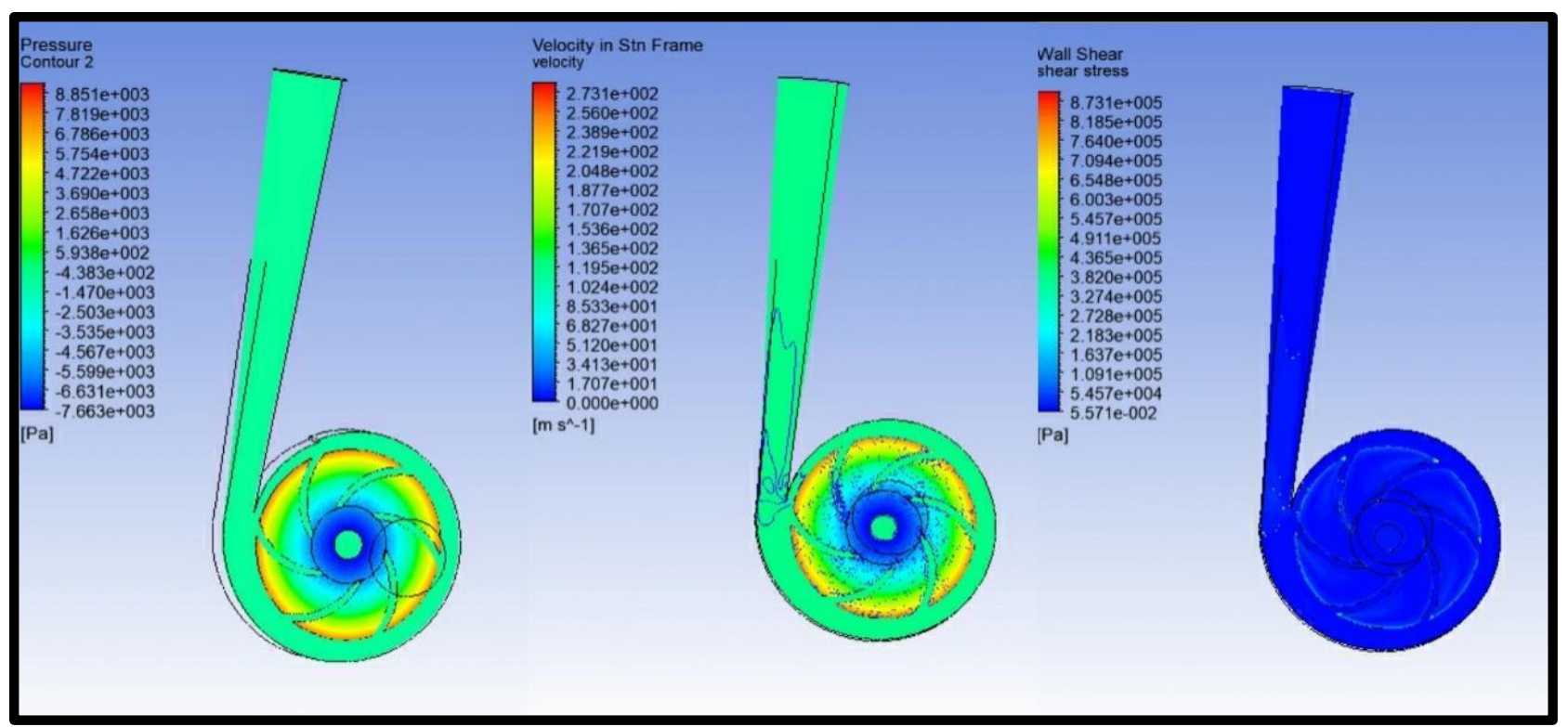

Figure 5.6: Impeller 3 pressure, velocity and shear stress profile

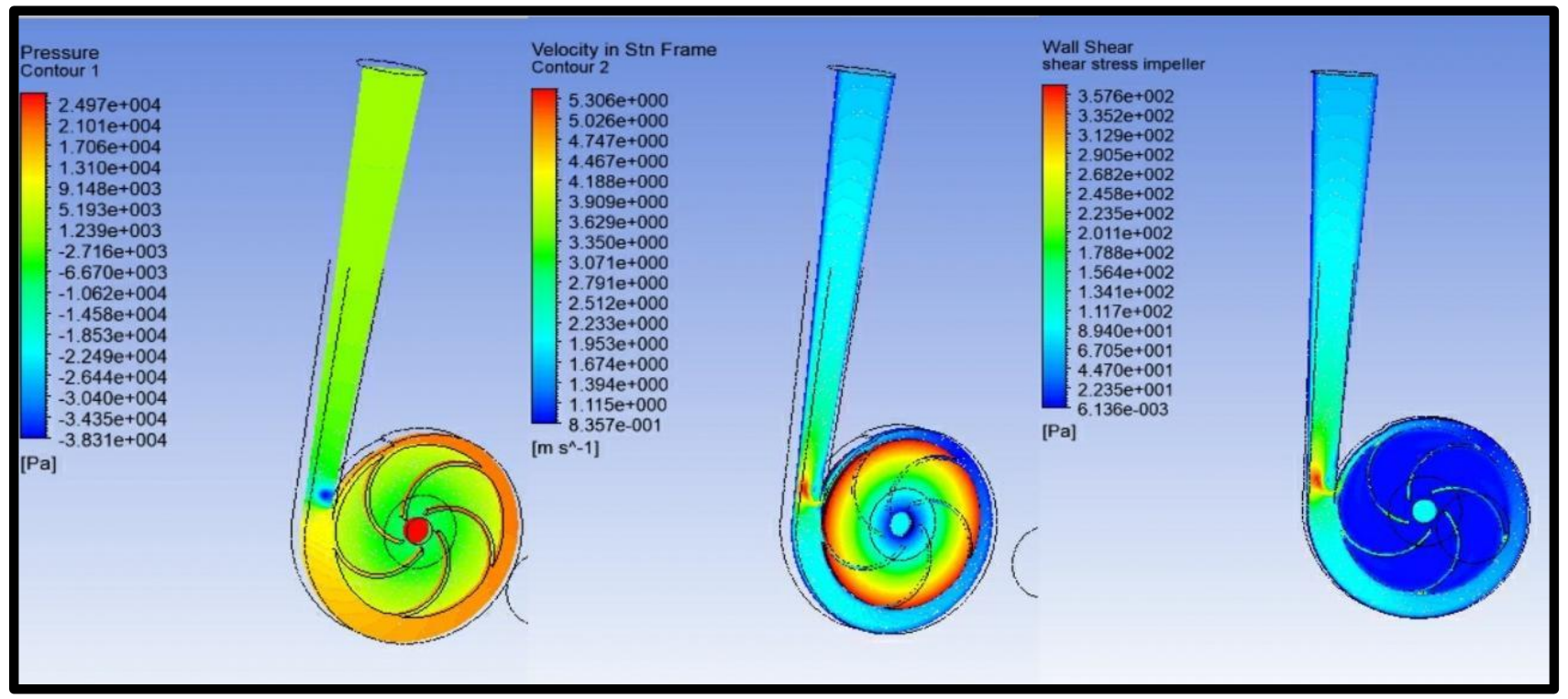

Figure 5.7: Impeller 4 pressure, velocity and shear stress profile 


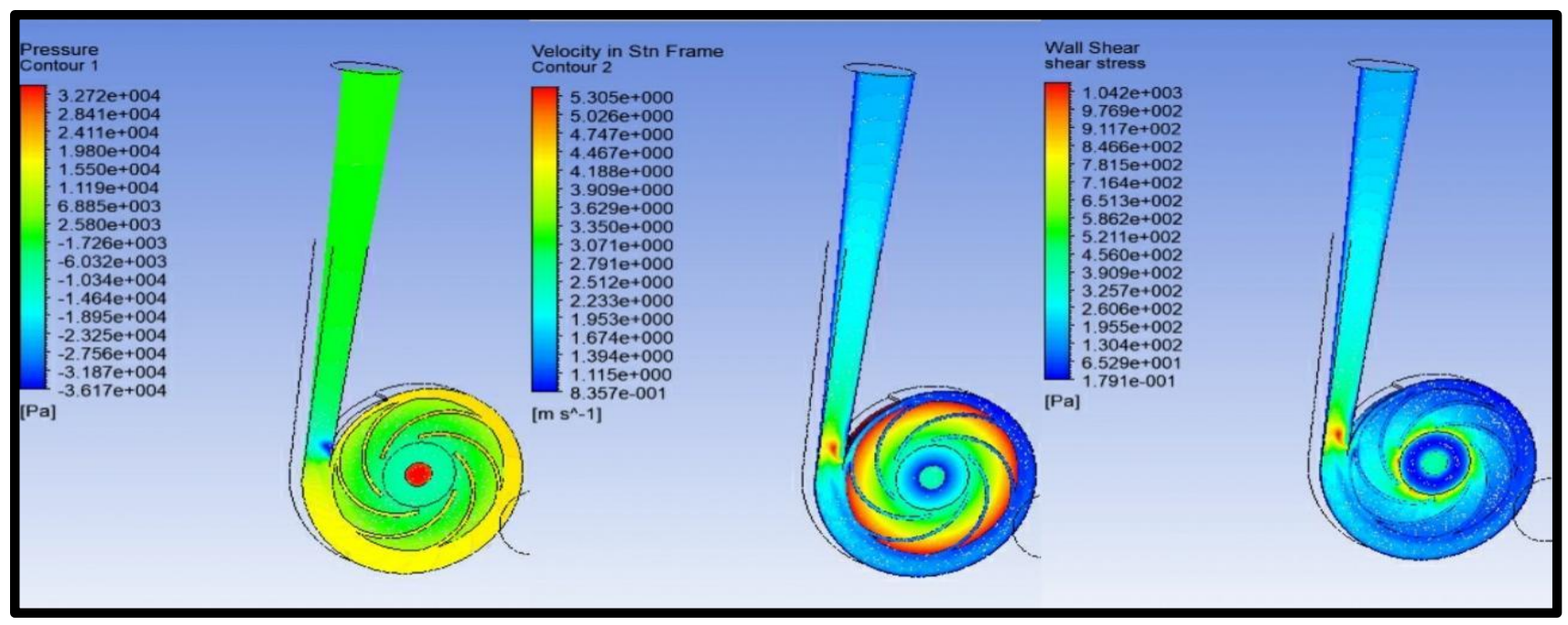

Figure 5.8: Impeller 5 pressure, velocity and shear stress profile

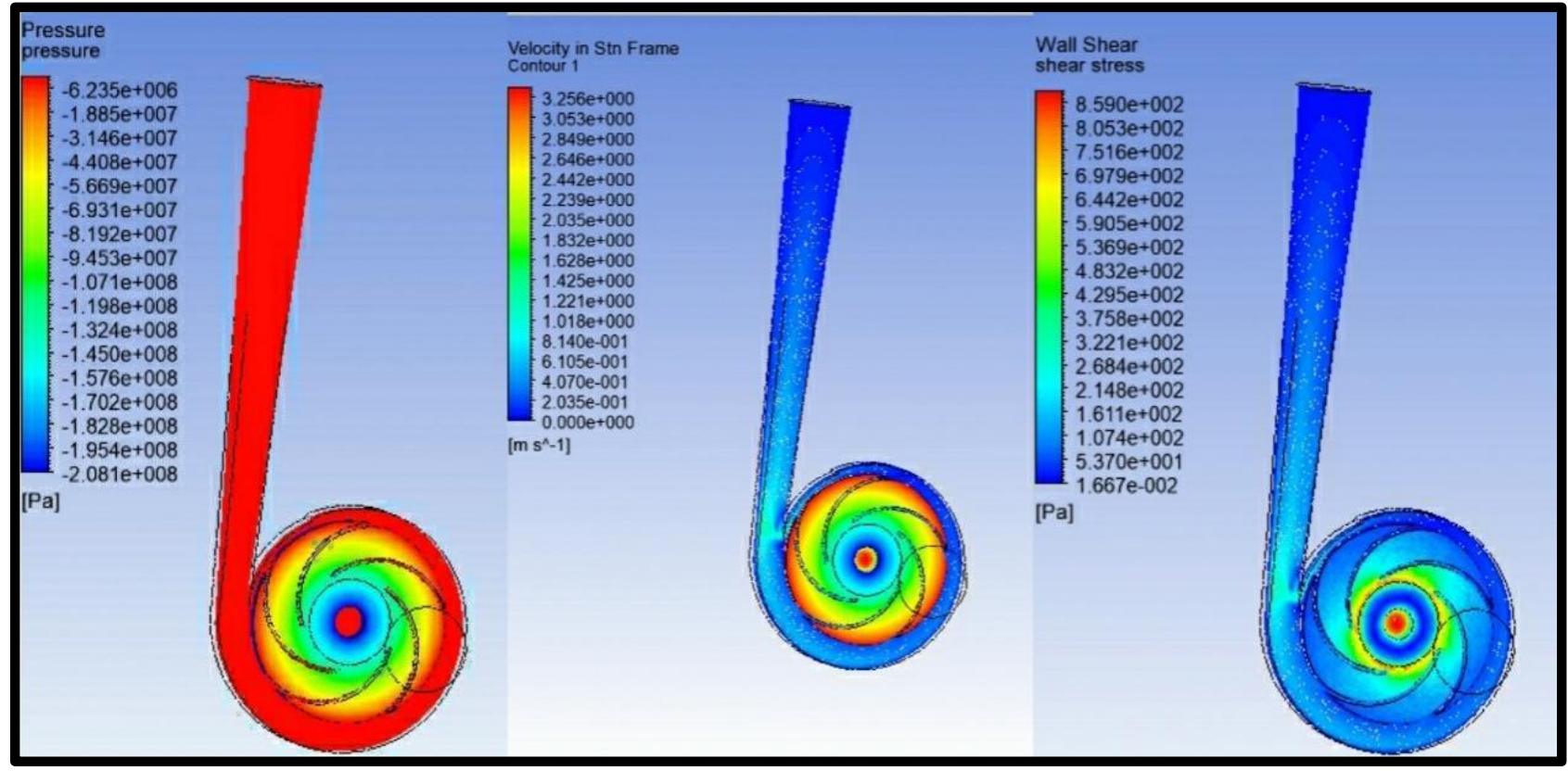

Figure 5.9: Impeller 6 pressure, velocity and shear stress profile

From [1], hemolysis range is between $150 \mathrm{~Pa}$ to $4000 \mathrm{~Pa}$. According to the above SS contour profiles almost every design has SS below this range. Therefore, it can be said that these designs are hemocompatible at this stage. 


\subsection{Conclusion}

The main focus of this Chapter was to find out and state which one of the previously 6 designed impellers of LVAD is to be considered as the perfect ideal model to be used as the design model for LVAD to be later be used for the simulation of the single sided RVAD and the Bi-VAD with the same characteristics as the ideal design model being considered for the LVAD. This Chapter consisted of the mathematical calculation being carried out as well as the graphical representations being shown to determine which one of the 6 designed impellers is to be considered as the perfect ideal model as already stated, and it is found out later in this very Chapter that Impeller1 which has been designed for $100^{\circ}$ wrap angle and 6 blades is considered as the perfect designed impeller to be be handed - down for the simulation of the RVAD as well as the Bi-VAD since it has the perfect combination of the finest value of Efficiency as well as the Head. Moreover, it has already been found out that impellers with value of Efficiency above $100 \%$ were opted out and not brought into consideration for the unacceptable value of Efficiency. Additionally, it has also been discussed that Impeller1 designed for 6 blades and $100^{\circ}$ wrap angle that has been taken as the best model design of impeller to be considered for the LVAD has an Efficiency value of $63.44 \%$. Although in [1], it has been discussed about how Impeller 2 in their case that has been designed with 6 blades and $110^{\circ}$ wrap angle gave the best and highest value of Efficiency among the other designed impellers with a value of less than $60 \%$. Hence, it can be concluded that by bringing certain modifications in the design of the impeller by keeping the number of blades to be 6 but changing the wrap angle from $110^{\circ}$ to $100^{\circ}$, the value of the obtained Efficiency of the impeller can be increased further as it has been observed in this Chapter upon simulation of Impeller 1 designed for 6 blades and $110^{\circ}$ wrap angle that gave a value of Efficiency of $63.44 \%$ under operating conditions of $0.3 \mathrm{~mm}$ best mesh size and a Mass Flow rate value of $0.088 \mathrm{~kg} / \mathrm{s}$. This designed impeller, Impeller1 is then being used for the simulation process of the RVAD and the Bi-VAD and from the obtained values of the Pressure difference and Torque obtained from the simulation, the Head and Efficiency calculation of the RVAD and Bi-VAD is then shown. 


\section{Chapter 6}

\section{Conclusion}

\subsection{Summary}

The primary motivation behind this research was to plan, design and simulate a working and proficient Bi-VAD model for the heart patients as a worry to the expanded worldwide heart issues. Initially the simulations were done successfully for 6 different LVAD designs and a number of factors were taken into consideration such as pressure difference, torque, Head pressure and Efficiency. The simulations were done for different mass Flow rates of the blood and operating conditions to observe the pressure rise and also the Efficiency was calculated for every stage for the better understanding of the pump performance in different heart conditions. The LVAD design with the highest Efficiency with a Head pressure within the range (100-150 $\mathrm{mmHg}$ ) [1]. at a selected average blood flow rate was assumed to be eligible for further simulation purposes. Subsequently, the simulations for RVAD and Bi-VAD were done with acceptable results. In the whole process, certain hemodynamic properties including parameters such as wall shear stress, pressure, velocity of the fluid were also reviewed as vital conditions and parameters required to solve the problem of thrombosis and hemolysis which is known to be a massive threat to the pump and may cause pump failure altogether. The wrap angle and blade numbers of the pump also projected a great impact on the design and simulation. In many surveys, wrap angle $120^{\circ}$ was found better in terms of Efficiency and pressure difference over smaller wrap angles such as $110^{\circ}$ and $100^{\circ}$. However, in this research wrap angle $100^{\circ}$ was giving promising results. Although blade number 6 was showing finer outcomes similar to previous research works.

\subsection{Future Goals}

Since human heart is a very sensitive and complex organ it requires more precision. The simulation results were acceptable only in theoretical terms. Moreover, there may be some error due to the software work function. For better and accurate evaluation this design could be made 
into a proto-type and run in a mock circulation loop with real human or animal blood. But there are some infrastructural deficiencies in Bangladesh to carry out this kind of research in practical. However, for the improvement of the design, a more extensive research can be done to increase the Efficiency and minimize the hemodynamic complication to the least. Additionally, there are scope to work on power consumption, battery life and other mechanical complexities. 


\section{References}

1. Bumrungpetch, J. (2016). MECHANISM DESIGN OF VENTRICULAR ASSIST DEVICE [Ebook]. Queensland University of Technology. Retrieved from https://eprints.qut.edu.au/94239/1/Jeerasit_Bumrungpetch_Thesis.pdf

2. K.Fukamachi and N.G. Smedira, "Smaller, Safer, Totally Implantable LVADs: Fact or Fantasy?”, ACC Current Journal Review, August 2008.

3. A.Acuna, A.G.Berman, F.W.Damen and A.Meyers, "Computational Fluid Dynamics of Vascular Disease in Animal Models", vol.140, August 2018.

4. E J.Birks, P D. Tansley, J. Hardy, R S. George, C T. Bowles, M.Burke and M H. Yacoub, "Left Ventricular Assist Device and Drug Therapy for the Reversal of Heart Failure",The New England Journal of Medicine, November 2, 2006.

5. B. T. Chan, C. W. Ong, E. Lim, N. A. Abu Osman, A. Al Abed, N. H. Lovell and S. Dokos, “ Simulation of Left Ventricle Flow Dynamics with Dilated Cardiomyopathy during the Filling Phase" in 34th Annual International Conference of the IEEE EMBS San Diego, California USA, 28 August - 1 September, 2012

6. K H. Fraser, M. E. Taskin, B P. Griffith, Zhongjun J. Wu, "The use of computational fluid dynamics in the development of ventricular assist device", 15 October 2018.

7. Behbahani, M., Behr, M., Hormes, M., Steinseifer, U., Arora, D., Coronado, O., \& Pasquali, M. (2009). A review of computational fluid dynamics analysis of blood pumps. European Journal of Applied Mathematics, 20(04), 363-397.doi: doi:10.1017/S0956792509007839

8.Kido, K., Hoshi, H., Watanabe, N., Kataoka, H., Ohuchi, K., Asama, J., . . . Takatani,S. (2006). Computational Fluid Dynamics Analysis of the Pediatric Tiny Centrifugal Blood Pump (TinyPump). Artificial Organs, 30(5), 392-399. doi:10.1111/j.1525-1594.2006.00231.x 
9. Masuzawa, T., Ohta, A., Tanaka, N., Qian, Y., \& Tsukiya, T. (2009). Estimation of changes in dynamic hydraulic force in a magnetically suspended centrifugal blood pump with transient computational fluid dynamics analysis. Journal of Artificial Organs, 12(3), 150-159. doi:10.1007/s10047-009-0459-2

10. Medvitz, R. B., Boger, D. A., Izraelev, V., Rosenberg, G., \& Paterson, E. G. (2011). Computational Fluid Dynamics Design and Analysis of a Passively Suspended Tesla Pump Left Ventricular Assist Device. Artificial Organs, 35(5), 522-533. doi: 10.1111/j.1525-1594.2010.01087.x

11. Selgrade, B. P., \& Truskey, G. A. (2012). Computational Fluid Dynamics Analysis to Determine Shear Stresses and Rates in a Centrifugal Left Ventricular Assist Device. Artificial Organs, 36(4), E89-E96. doi: 10.1111/j.1525-1594.2011.01416.x

12. Song, X., Throckmorton, A. L., Wood, H. G., Allaire, P. E., \& Olsen, D. B. (2004). Transient and Quasi-Steady Computational Fluid Dynamics Study of a Left Ventricular Assist Device. ASAIO journal, 50(5), 410-417.

13. Wu, J., Paden, B. E., Borovetz, H. S., \& Antaki, J. F. (2010). Computational Fluid Dynamics Analysis of Blade Tip Clearances on Hemodynamic Performance and Blood Damage in a Centrifugal Ventricular Assist Device. Artificial Organs, 34(5), 402-411. doi: 10.1111/j.1525-1594.2009.00875.x

14. Wells, R. E., \& Merrill, E. W. (1961). The variability of blood viscosity. The American Journal of Medicine, 31(4), 505-509. doi: 10.1016/0002-9343(61)90134-6

15. Wells, R. E., \& Merrill, E. W. (1962). Influence of flow properties of blood upon viscosity-hematocrit relationships. The Journal of clinical investigation, 41(8), 1591-1598. doi: 10.1172/JCI104617

16. Woodcock, J. P. (1976). Physical properties of blood and their influence on bloodflow measurement. Reports on Progress in Physics, 39, 65. doi:10.1088/0034-4885/39/1/002 
17. .Song, X., Wood, H. G., \& Olsen, D. (2004). Computational Fluid Dynamics (CFD) study of the 4th generation prototype of a continuous flow Ventricular Assist Device (VAD). Journal of biomechanical engineering, 126(2), 180. doi:10.1115/1.1688776

18. Continuity equation. (2019). Retrieved 10 August 2019, from https://en.wikipedia.org/wiki/Continuity equation?fbclid=IwAR3sfyk2mWIMQp0X5iwAZF7cUgIjX Gw39W9klBNCeW Zs7C6Af4iZ_C-hmA

19. Ferziger, J. H., \& Peric, M. (1996). Computational methods for fluid dynamics. New York: Springer-Verlag.

20. Tu, J., Yeoh, G.-H., \& Liu, C. (2013). Computational Fluid Dynamics: A Practical Approach. GB: Butterworth Heinemann.

21. George, W. K. (20013). Lectures in Turbulence for the 21st Century. Retrieved 4 June, 2015, from www.turbulence-online.com

22. Wilcox, D. C. (1998). Turbulence modeling for CFD. La Cãnada, Calif: DCW Industries

23. Davidson, L. (2003). An introduction to turbulence models. Department of Thermo and Fluid Dynamics, Chalmers University of Technology, Gothenburg, Sweden.

24. Davis, P., Rinehimer, A., \& Uddin, M. (2012). A comparison of RANS-based turbulence modeling for flow over a wall-mounted square cylinder. Paper presented at the $20^{\text {th }}$ Annual Conference of the CFD Society of Canada.

25. Menter, F. R. (1993). Zonal two equation k-turbulence models for aerodynamic flows. AIAA paper, 2906, 1993. 
26. Menter, F. R. (1994). Two-equation eddy-viscosity turbulence models for engineering applications. AIAA Journal, 32(8), 1598-1605. doi: 10.2514/3.12149

27. . Song, X., Wood, H. G., Day, S. W., \& Olsen, D. B. (2003). Studies of Turbulence Models in a Computational Fluid Dynamics Model of a Blood Pump.Artificial Organs, 27(10), 935-937. doi: 10.1046/j.1525-1594.2003.00025.x

28. Throckmorton, A. L., \& Untaroiu, A. (2008). CFD Analysis of a Mag-Lev Ventricular Assist Device for Infants and Children: Fourth Generation Design. ASAIO journal, 54(4), 423-431. doi:10.1097/MAT.0b013e31817efaa8

29. Giersiepen, M., Wurzinger, L. J., Opitz, R., \& Reul, H. (1990). Estimation of shear stress-related blood damage in heart valve prostheses--in vitro comparison of 25 aortic valves. The International journal of artificial organs, 13(5), 300-306.

30. Wurzinger, L., Opitz, R., \& Eckstein, H. (1986). Mechanical blood trauma: An overview. Angeiologie, 38, 81-97.

31. Taskin, M. E., Fraser, K. H., Zhang, T., Wu, C., Griffith, B. P., \& Wu, Z. J. (2012). Evaluation of Eulerian and Lagrangian Models for Hemolysis Estimation. ASAIO journal, 58(4), 363-372. doi: 10.1097/MAT.0b013e318254833b

32. Grigioni, M., Daniele, C., Morbiducci, U., D'Avenio, G., Benedetto, G. D., \& Barbaro, V. (2004). The Power-law Mathematical Model for Blood Damage Prediction: Analytical Developments and Physical Inconsistencies. Artificial Organs, 28(5), 467-475. doi: $10.1111 / \mathrm{j} .1525-1594.2004 .00015 . x$

33. Grigioni, M., Morbiducci, U., D’Avenio, G., Benedetto, G. D., \& Gaudio, C. D. (2005). A novel formulation for blood trauma prediction by a modified power-law mathematical model. Biomechanics and Modeling in Mechanobiology, 4(4), 249-260. doi: 10.1007/s10237005-0005-y 
34. Garon, A., \& Farinas, M.-I. (2004). Fast Three-dimensional Numerical Hemolysis Approximation. Artificial Organs, 28(11), 1016-1025. doi: 10.1111/j.1525-1594.2004.00026.x

35. Zhang, J., Gellman, B., Koert, A., Dasse, K. A., Gilbert, R. J., Griffith, B. P., \& Wu, Z. J.(2006). Computational and experimental evaluation of the fluid dynamics and hemocompatibility of the CentriMag blood pump. Artificial Organs, 30(3), 168-177.

36. Taskin, M. E., Fraser, K. H., Zhang, T., Gellman, B., Fleischli, A., Dasse, K. A., . .Wu, Z. J. (2010b). Computational characterization of flow and hemolytic performance of the UltraMag blood pump for circulatory support. Artificial Organs, 34(12), 1099. doi: 10.1111/j.15251594.2010.01017.x

37. Fraser, K. H., Zhang, T., Taskin, M. E., Griffith, B. P., \& Wu, Z. J. (2012). A quantitative comparison of mechanical blood damage parameters in rotary ventricular assist devices: shear stress, exposure time and hemolysis index. Journal of biomechanical engineering, 134(8), 081002.

38. Weidong Zhou, Zhimei Zhao, T. S. Lee and S. H.Winoto (2003), 'Investigation of Flow Through Centrifugal Pump Impellers Using Computational Fluid Dynamics', Fluid Mechanics Laboratory, Mechanical Engineering Department, National University of Singapore, Singapore.

39. Xinwei Song, Amy L. Throckmorton, Houston G. Wood, James F. Antaki and Don B. Olsen (2003), 'Computational Fluid Dynamics Prediction of Blood Damage in a Centrifugal Pump', Mechanical and Aerospace Engineering Department, Virginia Artificial Heart Institute, University of Virginia; Biomedical Engineering Department, Virginia Artificial Heart Institute, University of Virginia, Charlottesville, VA; McGowan Center for Artificial Organ Development, University of Pittsburgh, Pittsburgh, PA; and Utah Artificial Heart Institute, Salt Lake City, UT, U.S.A; 2003.

40. B. Jafarzadeh, A. Hajari, M.M. Alishahi , M.H. Akbari (2011), 'The flow simulation of a low-specific-speed high-speed centrifugal pump', 
a. High Performance Computing Center (HPCC), Department of Mechanical Engineering, School of Engineering, Shiraz University, Shiraz, Iran

b. Advanced Materials Research Center (AMRC), Materials \& Metallurgical Engineering Department, Iran University of Science \& Technology (IUST), Tehran 16844-1314, Iran

41. Venkatratnam dirisala, Asst prof. in MPES Group of institutions, Guntur, AP, India, 'Performance analysis of Centrifugal Pump by using CFD', International Journal of Mechanical Engineering and Technology (IJMET).

42. Anthony R. Curtas, Houston G. Wood, Paul E. Allaire, James C. Mcdaniel, Steven W. Day, and Don B. Olsen, "Computational Fluid Dynamics Modeling of Impeller Designs for the HeartQuest Left Ventricular Assist Device', ASAIO Journal 2002.

43. F.M. White, Fluid Mechanics, 7th Edition, McGraw-Hill, New York, 2011.

44. Mass flow rate. (2019). Retrieved 10 August 2019, from https://en.wikipedia.org/wiki/Mass_flow_rate?fbclid=IwAR2N1W1OPDSQwfvkmjo95EmHHN WP Uqh iXbIUSUaocX2Q-MsLbX-tjg7EM

45. Xian-Hua, L., Shu-Jia, Z., Bao-Lin, Z., \& Qing-Bo, H. (2006). "The Study of the $k-$ $\varepsilon$ Turbulence Model for Numerical Simulation of Centrifugal Pump”, 2006 7th International Conference on Computer-Aided Industrial Design and Conceptual Design. doi: 10.1109/caidcd.2006.329435

46. Ostadfar, A. (2017). Hemolysis in Medical Devices. International Journal of Biosensors \& Bioelectronics, 2(5). 


\section{Appendix A:}

\section{Manual Brochure for ANSYS Fluent version 18.1 software:}

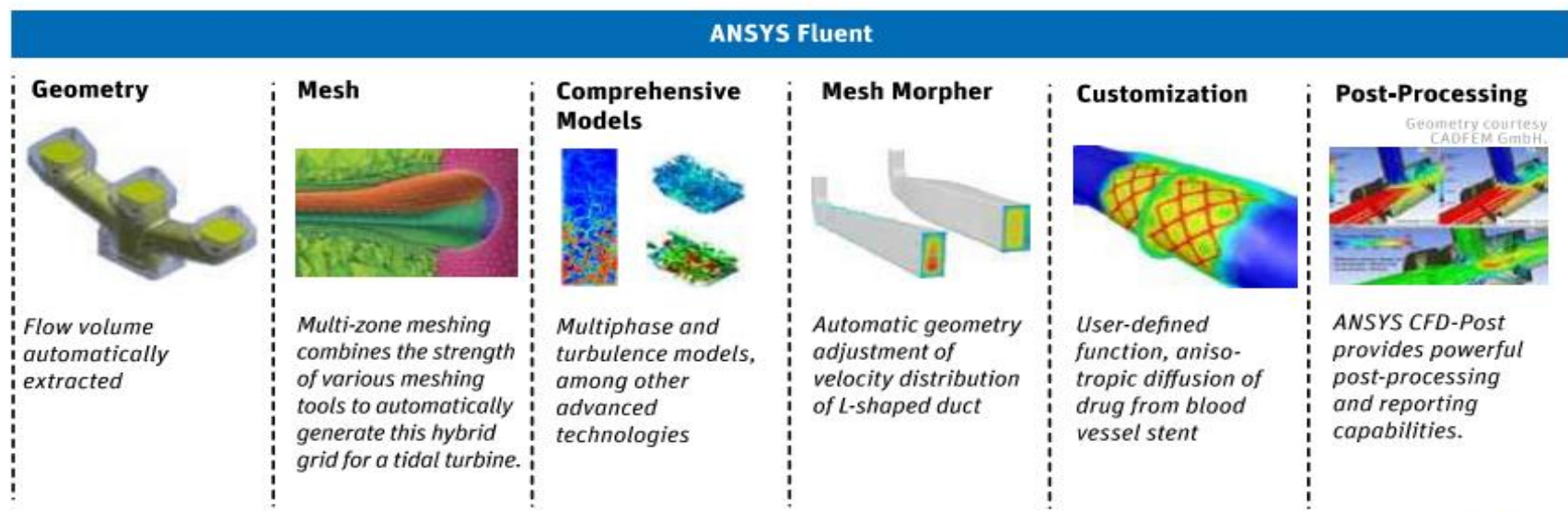

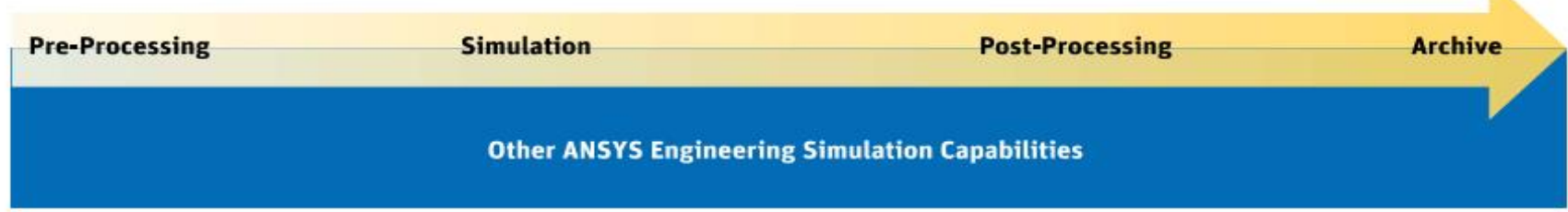

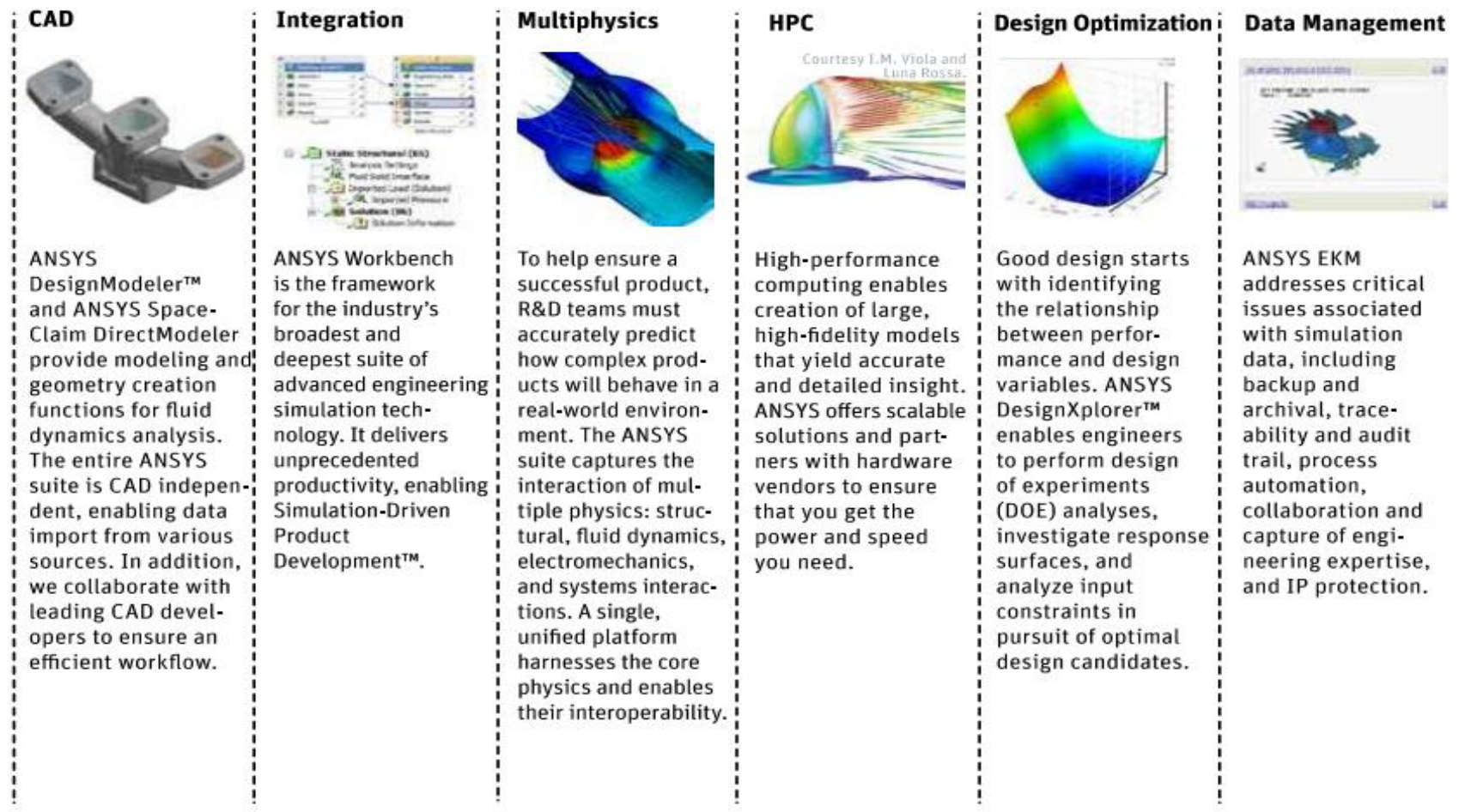




\section{Appendix B:}

Manual Brochure for SOLIDWORKS 2018 software:

Overview of SOLIDWORKS ${ }^{\oplus}$ and the User Interface

SOLIDWORKS ${ }^{\circledast 2018}$ Tutorial

\section{Summary}

The SOLIDWORKS 2018 User Interface and CommandManager consist of the following main options: Menu bar toolbar, Menu bar menu, Drop-down menus, Context toolbars, Consolidated fly-out menus, System feedback icons, Confirmation Corner and Heads-up View toolbar.

The default CommandManager Part tabs control the display of the Features, Sketch, Evaluate, DimXpert and SOLIDWORKS Add-Ins toolbars.

The FeatureManager consists of five default tabs:

- FeatureManager design tree.

- PropertyManager.

- ConfigurationManager.

- DimXpertManager.

- DisplayManager.

You learned about creating a new SOLIDWORKS part and opening an existing SOLIDWORKS part along with using the Rollback bar to view the sketches and features.

You also learned about SOLIDWORKS Help, SOLIDWORKS Tutorials and basic mouse movements to manipulate your SOLIDWORKS model.

=̀́

Opening a SOLIDWORKS document from an earlier release can take extra time. After you open and save a file, subsequent opening time returns to normal. Use the SOLIDWORKS Task Scheduler (SOLIDWORKS

Professional) to convert multiple files from an earlier version to the SOLIDWORKS 2018 format. Click Windows Start > All Apps > SOLIDWORKS 2018 > SOLIDWORKS Tools 2018 > SOLIDWORKS Task Scheduler.

Templates are part, drawing and assembly documents which include user-defined parameters. Open a new part, drawing or assembly. Select a template for the new document.

- Parts. The Parts default template is located in the C: ProgramData $\backslash$ SolidWorks $\backslash$ SOLIDWORKS $2018 \backslash$ templates $\backslash$ Part.prtdot folder.

- Assemblies. The Assemblies default template is located in the C: ProgramData $\backslash$ SolidWorks $\backslash$ SOLIDWORKS $2018 \backslash$ templates $\backslash$ Assembly.asmdot folder.

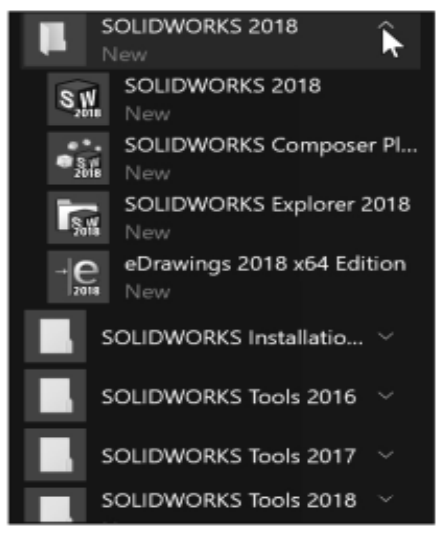


- Drawings. The Drawings default template is located in the C:ProgramData|SolidWorks $\backslash$ SOLIDWORKS 2018 \templates\Drawing.drwdot folder.

$=2$

If you modify a document property from an Overall drafting standard, a modify message is displayed as illustrated.

For commercial users, SOLIDWORKS Model Based Definition

Overall drafting standard ANSI-MODIFIED

Derived from: ANSI (MBD) is a separate application. For education users, SOLIDWORKS MBD is included in the SOLIDWORKS Education Edition as an Add In.

Redeem your code on the inside face of the book. View the provided videos and models to enhance the user experience.

In Chapter 2, obtain the working familiarity of the following SOLIDWORKS sketch and feature tools: Line, Circle, Centerpoint Straight Slot, Smart Dimension, Extruded Boss/Base, Extruded Cut and Linear Pattern.

Create three individual parts: AXLE, SHAFTCOLLAR and FLATBAR.

Create the assembly, LINKAGE using the three

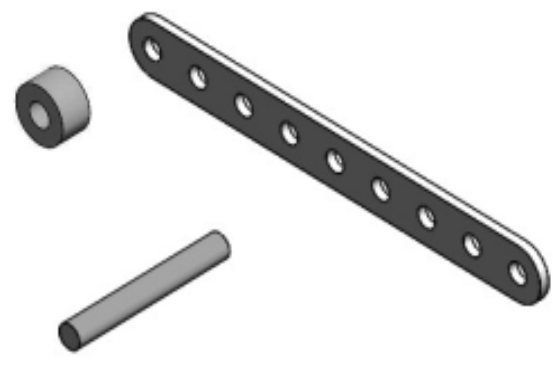
created parts and the downloaded sub-assembly AirCylinder.
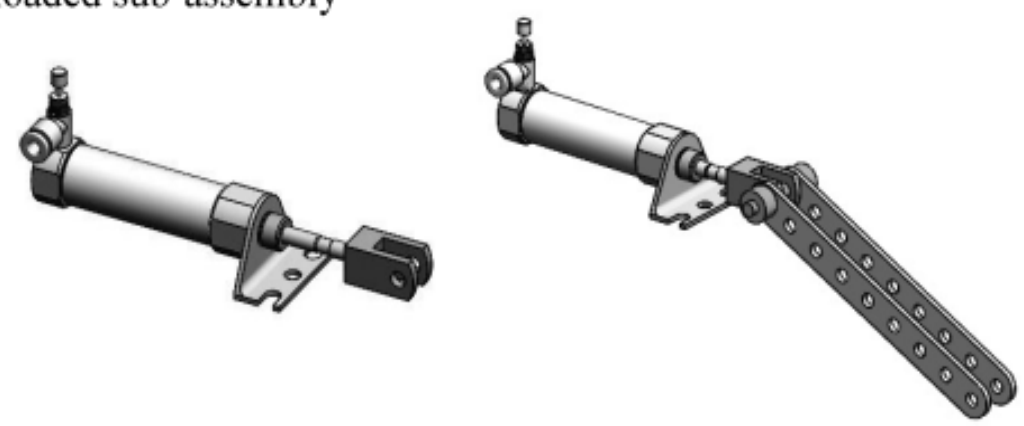